\title{
A THEORETICAL AND EXPERIMENTAL INVESTIGATION OF MODULATION SIDEBANDS OF PLANETARY GEAR SETS
}

\author{
DISSERTATION \\ Presented in Partial Fulfillment of the Requirements for \\ the Degree Doctor of Philosophy in the Graduate \\ School of The Ohio State University
}

By

Murat Inalpolat, M.S.

$* * * * *$

The Ohio State University

2009

Dissertation Committee:

Professor Ahmet Kahraman, Adviser

Approved by

Professor Rajendra Singh

Professor Chia-Hsiang Menq

Professor Dennis Guenther

Adviser

Mechanical Engineering Graduate Program 



\begin{abstract}
A pair of mathematical models is proposed in this study to investigate the modulation sidebands that routinely exist in measured vibration spectra from planetary transmissions. As the first of these models, a simplified semi-analytical model is proposed to describe the mechanisms leading to modulation sidebands of planetary gear sets due to rotation of the planet carrier. The model includes key system parameters such as number of planets, planet position angles, and planet phasing relationships defined by the position angles and the number of teeth of the gears. The model is used to simulate a wide range of gear sets to show that they can be classified in five distinct groups based on their sideband behavior in terms of their frequencies and amplitudes. A special experimental planetary gear set-up is developed and planetary gear sets from of three of these five groups are procured. A methodology is developed to demonstrate modulation sidebands from the ring (internal) gear radial acceleration measurements. For each case, sets of ring gear acceleration measurements at various speed and torque conditions are presented to demonstrate rich sideband activity that agrees well with the model predictions.
\end{abstract}

The second model proposed in this study is a non-linear time-varying dynamic model that includes periodically varying gear mesh stiffnesses and non-linearities due to 
tooth separations. This model employed an excitation formulation that captures the impact of various gear manufacturing errors such that the predicted dynamic gear mesh force spectra contain separate sets of sidebands at unique frequencies to correspond to these errors. This model is further combined with the first model to incorporate the effects of carrier rotation as well. Comparison to the measured spectra are used to demonstrate the capability of the model in predicting the sidebands of a planetary gear set with both gear manufacturing errors and a rotating carrier. At the end, based on results of the parametric studies and experiments, general rules on modulation sidebands of planetary gear sets are proposed. 
Dedicated to my wife

Berna 


\section{ACKNOWLEDGMENTS}

I express my sincere gratitude to Professor Ahmet Kahraman for his guidance and support throughout this research. I am also thankful to Professor Rajendra Singh, Professor Chia-Hsiang Menq and Professor Dennis Guenther for serving on the dissertation committee.

I would like to thank Dr. Avinash Singh of General Motors Powertrain for allowing the use of the text fixtures and gear specimens in this study. In addition, I am grateful to Jonny Harianto, Sam Shon and Gary Gardner for providing technical and administrative support on the way.

I want to sincerely thank my family for their continuous support and encouragement without which this work would not have been possible. Finally, I would like to thank my wife, Berna, for her patience and faith in me throughout this endeavor. 
VITA

September 28, 1978......................Born - Istanbul, Turkey

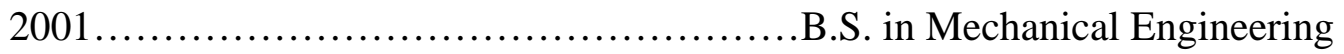
Dokuz Eylul University, Turkey

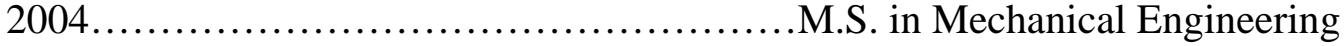
Middle East Technical University, Turkey

2009......................................... in Mechanical Engineering The Ohio State University

2005-2009...................................Graduate Research Associate The Ohio State University

\section{PUBLICATIONS}

1. M. Inalpolat, A. Kahraman, "A theoretical and experimental investigation of modulation sidebands of planetary gear sets” Journal of Sound and Vibration, 323 (2009) 677-696.

\section{FIELDS OF STUDY}

Major Field: Mechanical Engineering 


\section{TABLE OF CONTENTS}

\section{Page}

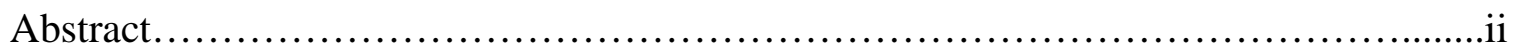

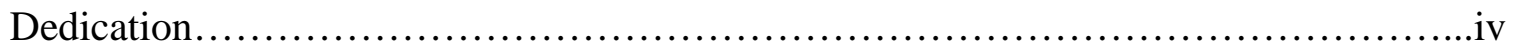

Acknowledgments......................................................................

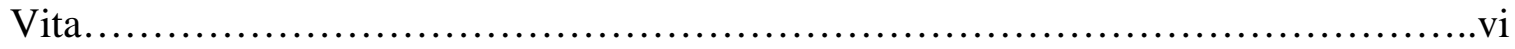

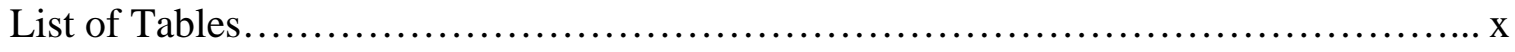

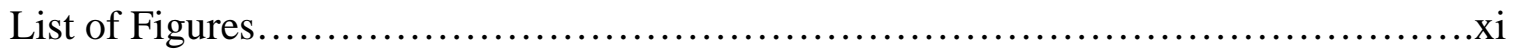

Chapters:

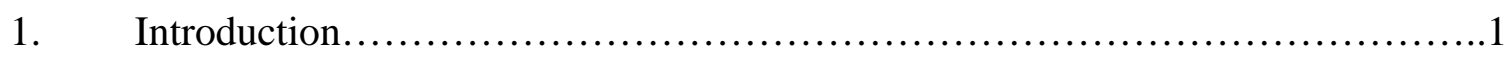

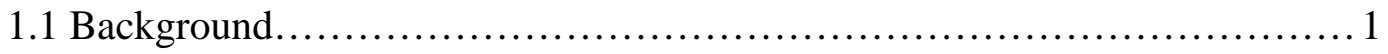

1.2 Motivation..................................................................

1.3 Thesis Objectives and Scope........................................... 5

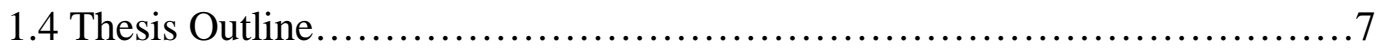

2. Planetary Gear Set Modulation Sideband Experiments...........................11

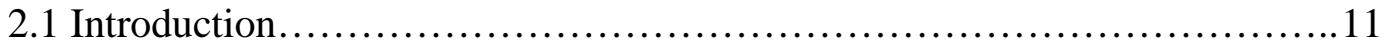

2.2 Experimental Set-up..................................................12

2.3 Test Gear Sets and Test Matrix..........................................14

2.4 Instrumentation, Data Collection and Data Analysis........................16 
2.5 Experimental Results.................................................... 18

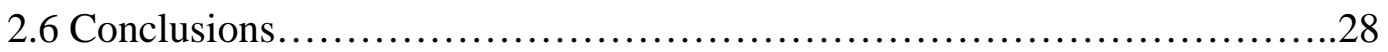

3. Modulation Sidebands Due to a Rotating Carrier................................31

3.1 Introduction....................................................................

3.2 Analytical Model.......................................................

3.3 Classification of Planetary Gear Sets based on Their Sideband Behavior......42

3.4 Experimental Verification of the Modulation Model..........................57

3.4.1 Categorization of the Experimental Planetary Gear Sets based on Sideband Patterns........................................57

3.4.2 Experimental Results......................................... 59

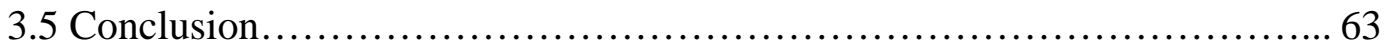

4. Modulation Sidebands of a Planetary Gear Set with Manufacturing Errors...... 70

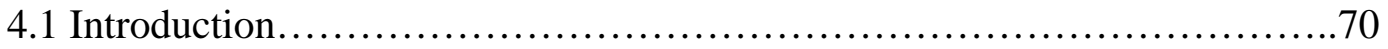

4.2 Prediction of Modulation Sidebands under Dynamic Conditions.............. 74

4.2.1 Dynamic Model of a Planetary Gear Set.......................... 74

4.2.2 Formulation of Modulations of the Excitations

Due to Manufacturing Errors.......................................85

4.2.3 Formulation of Modulations due to Rotating Carrier................ 91

4.3 An Example Analysis of Modulation Sidebands of a Planetary

Gear Set with Manufacturing Errors.....................................93

4.3.1 Planetary Gear Set Modulations under Off-resonance Conditions.........................................94 
4.3.2 Planetary Gear Set Modulations Near a Resonance............... 114

4.4 Experimental Validation........................................117

4.5 Conclusions........................................................ 123

5. Conclusions and Recommendations................................ 127

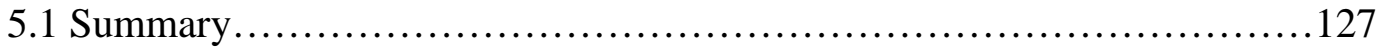

5.2 Conclusions and Contributions....................................129

5.3 Recommendations for Future Work .................................133 


\section{LIST OF TABLES}

Table Page

4.1 Basic design parameters of the example planetary gear set....................95 


\section{LIST OF FIGURES}

Figure

Page

1.1 Schematic of a 4-planet planetary gear set showing its main components: a sun gear, a ring gear, four identical planet gears and a planet carrier

2.1 (a) Cross-section of the back-to-back planetary test fixtures, and (b) a view of the test set-up on a transmission dynamometer [2.1]

2.2 (a) A set of test gears and (b) 3, 4 and 5 planet carriers used in this study.....

2.3 A view of the accelerometers mounted radially on the outside surface of the test ring gear....

2.4 Examples of measured $a(t)$ at $\Omega_{s}=3200 \mathrm{rpm}$ from

(a) a 3-planet gear set $(N=3)$ at $T_{S}=800 \mathrm{Nm}$,

(b) a 4-planet gear set $(N=4)$ at $T_{S}=1000 \mathrm{Nm}$ and

(c) a 5-planet gear set $(N=5)$ at $T_{S}=1000 \mathrm{Nm}$.

2.5 An example measured acceleration frequency spectrum from a 3-planet gear set gear set at $T_{S}=800 \mathrm{Nm}$ and $\Omega_{S}=3200 \mathrm{rpm}$.

2.6 Measured acceleration spectrum from a 3-planet gear set at $T_{S}=400 \mathrm{Nm}$ and $\Omega_{\mathrm{S}}=500 \mathrm{rpm}$

2.7 Measured acceleration spectrum from a 3-planet gear set with a planet pin position error acquired at $T_{S}=400 \mathrm{Nm}$ and $\Omega_{S}=500 \mathrm{rpm}$

2.8 Measured acceleration spectrum from a 4-planet gear set at $T_{S}=800 \mathrm{Nm}$ and $\Omega_{s}=500 \mathrm{rpm}$

2.9 Measured acceleration spectrum from a 4-planet gear set with a planet pin position error acquired at $T_{S}=600 \mathrm{Nm}$ and $\Omega_{S}=500 \mathrm{rpm} \ldots \ldots . .26$ 
2.10 Measured acceleration spectrum from a 5-planet gear set at $T_{S}=800 \mathrm{Nm}$ and $\Omega_{S}=500 \mathrm{rpm}$

3.1 A schematic showing the components of a 4-planet planetary gear set and an accelerometer mounted on the ring gear....

3.2 A schematic illustration of $F_{i}(t), w_{i}(t)$ and $a(t)$ for a gear set having $N=3$ and $Z_{r}=25$

3.3 Theoretical $A(\omega)$ spectra for four different in-phase and equally-spaced planetary gear sets; (a) $N=3, Z_{r}=123$ and $Z_{s}=72$, (b) $N=4, Z_{r}=124$ and $Z_{s}=72$, (c) $N=5, Z_{r}=125$ and $Z_{s}=70$, and (d) $N=6, Z_{r}=126$ and $Z_{s}=72$

3.4 Theoretical $A(\omega)$ spectra for four different sequentially-phased and equally-spaced planetary gear sets; (a) $N=3, Z_{r}=125$ and $Z_{s}=73$, (b) $N=4, Z_{r}=126$ and $Z_{s}=74$, (c) $N=5, Z_{r}=126$ and $Z_{s}=74$, and (d) $N=6, Z_{r}=122$ and $Z_{s}=70$

3.5 Theoretical $A(\omega)$ spectra for four different in-phase and unequally-spaced planetary gear sets; (a) $N=3, Z_{r}=126$ and $Z_{s}=72$ with $\psi_{i}=0, \frac{2 \pi}{3}$ and $1 . \overline{2} \pi$, (b) $N=4, Z_{r}=120$ and $Z_{s}=72$ with $\psi_{i}=0,0.4167 \pi, \pi$ and $1.4167 \pi$, (c) $N=5, Z_{r}=145$ and $Z_{s}=75$ with $\psi_{i}=0,0.4276 \pi, 0.8276 \pi, 1.2 \pi, 1.6 \pi$, and (d) $N=6$, $Z_{r}=130, Z_{s}=70, \psi_{i}=0,0.4 \pi, \quad 0.74 \pi, \pi, 1.37 \pi$ and $1.74 \pi$

3.6 Theoretical $A(\omega)$ spectra for four different sequentially-phased and unequally-spaced planetary gear sets;
(a) $N=3, Z_{r}=125, Z_{s}=73, \psi_{i}=0,0 . \overline{5} \pi, 1 . \overline{4} \pi$,
(b) $N=5, Z_{r}=125, Z_{s}=73, \psi_{i}=0,0.404 \pi, 0.798 \pi, 1.202 \pi$ and $1.596 \pi$,
(c) $N=4, Z_{r}=126, Z_{s}=74$, and $\psi_{i}=0,0.54 \pi, 0.97 \pi$, and $1.57 \pi$, and (d) $N=6, Z_{r}=176, Z_{S}=74$, and $\psi_{i}=0,0.376 \pi, 0.704 \pi, \pi, 1.376 \pi$ and $1.68 \pi$ 53 
3.7 Theoretical $A(\omega)$ spectra for four different arbitrarily-phased and unequally-spaced planetary gear sets; (a) $N=3, Z_{r}=125, Z_{s}=73$

and $\psi_{i}=0,0 . \overline{55} \pi$ and $1.384 \pi$, (b) $N=4, Z_{r}=125, Z_{s}=73$

and $\psi_{i}=0,0.505 \pi, \pi$ and $1.505 \pi$, (c) $N=5, Z_{r}=127, Z_{s}=73$

and $\psi_{i}=0,0.42 \pi, 0.83 \pi, 1.24 \pi$ and $1.65 \pi$, and

(d) $N=6, Z_{r}=125, Z_{s}=73$, and

$\psi_{i}=0,0 . \overline{34} \pi, 0 . \overline{68} \pi, 1 . \overline{03} \pi, 1 . \overline{34} \pi, 1 . \overline{66} \pi$ 56

3.8 Examples of measured $A(\omega)$ order spectra for the Gear Set A at different torque and speed conditions; (a) $T_{S}=500 \mathrm{Nm}, \Omega_{\mathrm{s}}=400 \mathrm{rpm}$,

(b) $T_{S}=600 \mathrm{Nm}, \Omega_{s}=500 \mathrm{rpm}$, (c) $T_{S}=200 \mathrm{Nm}, \Omega_{s}=200 \mathrm{rpm}$, and (d) $T_{S}=1000 \mathrm{Nm}, \Omega_{S}=500 \mathrm{rpm}$.

3.9 Examples of measured $A(\omega)$ order spectra for the Gear Set B at different toque and speed conditions; (a) $T_{S}=400 \mathrm{Nm}, \Omega_{s}=500 \mathrm{rpm}$,

(b) $T_{s}=500 \mathrm{Nm}, \Omega_{s}=500 \mathrm{rpm}$, (c) $T_{s}=800 \mathrm{Nm}, \Omega_{s}=400 \mathrm{rpm}$, and (d) $T_{S}=1000 \mathrm{Nm}, \Omega_{s}=500 \mathrm{rpm}$

3.10 Examples of measured $A(\omega)$ order spectra for the Gear Set $\mathrm{C}$ at different torque and speed conditions; (a) $T_{S}=200 \mathrm{Nm}, \Omega_{S}=800 \mathrm{rpm}$,

(b) $T_{S}=400 \mathrm{Nm}, \Omega_{s}=500 \mathrm{rpm}$, (c) $T_{s}=600 \mathrm{Nm}, \Omega_{s}=500 \mathrm{rpm}$, and (d) $T_{S}=1000 \mathrm{Nm}, \Omega_{S}=200 \mathrm{rpm}$.

4.1 Components and basic geometric parameters of a simple planetary gear set (only one planet branch is shown).

4.2 Dynamic models of (a) a sun-planet pi pair, (b) a ring-planet pi pair, and (c) a carrier-planet pi pair.

4.3 Predicted (a) $F_{s p 1}(\omega)$ and (b) $F_{r p 1}(\omega)$ spectra for the example planetary gear set under the influence of AM due to planet-1 run-out error with $\beta_{p 1}=0.034, \phi_{\beta p 1}=3 \pi / 2, \vartheta_{p 1}=0.033, \phi_{\vartheta p 1}=\pi / 2$, $\kappa_{p 1}=0.055, \phi_{\kappa p 1}=3 \pi / 2, \tau_{p 1}=0.049$ and $\phi_{\tau p 1}=\pi / 2$. .97 
4.4 Predicted $A(\omega)$ spectra for the example planetary gear set with a rotating carrier under the influence of AM due to planet-1 run-out error with $\beta_{p 1}=0.034, \phi_{\beta p 1}=3 \pi / 2, \vartheta_{p 1}=0.033, \phi_{\vartheta p 1}=\pi / 2$, $\kappa_{p 1}=0.055, \phi_{\kappa p 1}=3 \pi / 2, \tau_{p 1}=0.049$ and $\phi_{\tau p 1}=\pi / 2$

4.5 Predicted (a) $F_{s p 1}(\omega)$ and (b) $F_{r p 1}(\omega)$ spectra for the example planetary gear set under the influence of AM due to sun gear run-out error with $\beta_{s i}=0.0338(i \in[1,4]), \phi_{\beta s 1}=\pi / 2, \phi_{\beta s 2}=0, \phi_{\beta s 3}=3 \pi / 2$, $\phi_{\beta s 4}=\pi$ and $\kappa_{s i}=0.0547(i \in[1,4]), \phi_{\kappa s 1}=\pi / 2, \phi_{\kappa s 2}=0$, $\phi_{\kappa s 3}=3 \pi / 2$ and $\phi_{\kappa s 4}=\pi$

4.6 Predicted $A(\omega)$ spectra for the example planetary gear set with a rotating carrier under the influence of AM due to sun gear run-out error with $\beta_{s i}=0.0338(i \in[1,4]), \phi_{\beta s 1}=\pi / 2, \phi_{\beta s 2}=0, \phi_{\beta s 3}=3 \pi / 2$, $\phi_{\beta s 4}=\pi$ and $\kappa_{s i}=0.0547(i \in[1,4]), \phi_{\kappa s 1}=\pi / 2, \phi_{\kappa s 2}=0$, $\phi_{\kappa s 3}=3 \pi / 2$ and $\phi_{\kappa s 4}=\pi$

4.7 Predicted (a) $F_{s p 1}(\omega)$ and (b) $F_{r p 1}(\omega)$ spectra for the example planetary gear set under the influence of AM due to planet-1, sun and ring gear run-out errors. $\hat{\beta}_{r}=\hat{\kappa}_{r}=2.0(10)^{-5}(i \in[1,4])$, $\phi_{\hat{\beta} r 1}=\phi_{\hat{\tau} r 1}=\frac{3 \pi}{2}, \phi_{\hat{\beta} r 2}=\phi_{\hat{\kappa} r 2}=0, \phi_{\hat{\beta} r 3}=\phi_{\hat{\kappa} r 3}=\frac{\pi}{2}$, and $\phi_{\hat{\beta} r 4}=\phi_{\hat{\kappa} r 4}=\pi$, and sun and planet related parameters are as in Fig. 4.4 and 4.6 103 
4.8 Predicted $A(\omega)$ spectra for the example planetary gear set with a rotating carrier under the influence of AM due to planet-1, sun and ring gear run-out errors. $\hat{\beta}_{r}=\hat{\kappa}_{r}=2.0(10)^{-5}$ $(i \in[1,4]), \phi_{\hat{\beta} r 1}=\phi_{\hat{\tau} r 1}=\frac{3 \pi}{2}, \phi_{\hat{\beta} r 2}=\phi_{\hat{\kappa} r 2}=0, \phi_{\hat{\beta} r 3}=\phi_{\hat{\kappa} r 3}=\frac{\pi}{2}$, and $\phi_{\hat{\beta} r 4}=\phi_{\hat{\kappa} r 4}=\pi$, and sun and planet related parameters are as in Fig. 4.4 and 4.6

4.9 Predicted (a) $F_{s p 1}(\omega)$ and (b) $F_{r p 1}(\omega)$ spectra for the example planetary gear set when only FM are present due to planet-1 run-out with $\hat{\beta}_{p 1}=\hat{\vartheta}_{p 1}=\hat{\kappa}_{p 1}=\hat{\tau}_{p 1}=8.5(10)^{-5}, \phi_{\hat{\beta} p 1}=3 \pi / 2$, $\phi_{\hat{\vartheta} p 1}=\pi / 2, \phi_{\hat{\kappa} p 1}=3 \pi / 2$ and $\phi_{\hat{\tau} p 1}=\pi / 2$

4.10 Predicted $A(\omega)$ spectrum for the example planetary gear set when only FM are present due to planet-1 run-out with $\hat{\beta}_{p 1}=\hat{\vartheta}_{p 1}=\hat{\kappa}_{p 1}=\hat{\tau}_{p 1}=8.5(10)^{-5}, \phi_{\hat{\beta} p 1}=3 \pi / 2$, $\phi_{\hat{\vartheta} p 1}=\pi / 2, \phi_{\hat{\kappa} p 1}=3 \pi / 2$ and $\phi_{\hat{\tau} p 1}=\pi / 2$. (a) A zoomed in view in the vicinity of the fundamental gear mesh frequency and (b) the same spectrum with a wider frequency range. 108

4.11 Predicted (a) $F_{s p 1}(\omega)$ and (b) $F_{r p 1}(\omega)$ spectra for the example planetary gear set when only FM are present due to sun gear run-out with $\hat{\beta}_{s}=\hat{\kappa}_{s}=3.0(10)^{-5}, i \in[1,4], \phi_{\hat{\beta} s 1}=\phi_{\hat{\kappa} s 1}=\frac{\pi}{2}$, $\phi_{\hat{\beta} s 2}=\phi_{\hat{\kappa} s 2}=0, \phi_{\hat{\beta} s 3}=\phi_{\hat{\kappa} s 3}=\frac{3 \pi}{2}$ and $\phi_{\hat{\beta} s 4}=\phi_{\hat{\kappa} s 4}=\pi$ .109 
4.12 Predicted $A(\omega)$ spectrum for the example planetary gear set when only FM are present due to sun gear run-out with parameters specified in Fig. 4.11. (a) A zoomed in view in the vicinity of the fundamental gear mesh frequency and (b) the same spectrum with a wider frequency range

4.13 Predicted (a) $F_{s p 1}(\omega)$ and (b) $F_{r p 1}(\omega)$ spectra for the example planetary gear set when only FM are present due to planet-1, sun gear and ring gear run-out errors. $\hat{\beta}_{r}=\hat{\kappa}_{r}=2.0(10)^{-5}$ $(i \in[1,4]), \phi_{\hat{\beta} r 1}=\phi_{\hat{\tau} r 1}=\frac{3 \pi}{2}, \phi_{\hat{\beta} r 2}=\phi_{\hat{\kappa} r 2}=0, \phi_{\hat{\beta} r 3}=\phi_{\hat{\kappa} r 3}=\frac{\pi}{2}$, and $\phi_{\hat{\beta} r 4}=\phi_{\hat{\kappa} r 4}=\pi$ and sun and planet related parameters are as in Fig. 4.10 and 4.12

4.14 Predicted $A(\omega)$ spectrum for the example planetary gear set when only FM when only FM are present due to planet-1, sun gear and ring gear run-out errors with parameters specified in Fig. 4.13. (a) A zoomed in view in the vicinity of the fundamental gear mesh frequency and (b) the same spectrum with a wider frequency range.

4.15 Predicted (a) $F_{s p 1}(\omega)$ and (b) $F_{r p 1}(\omega)$ spectra for the example planetary gear set under the influence of AM due to planet-1 run-out error with $\beta_{p 1}=0.034, \phi_{\beta p 1}=3 \pi / 2, \vartheta_{p 1}=0.033, \phi_{\vartheta p 1}=\pi / 2$, $\kappa_{p 1}=0.055, \phi_{\kappa p 1}=3 \pi / 2, \tau_{p 1}=0.049, \phi_{\tau p 1}=\pi / 2$ and $\omega_{m}=151.2 \mathrm{rad} / \mathrm{s}$ 
4.16 Predicted $A(\omega)$ spectrum for the example planetary gear set under the influence of AM due to planet-1 run-out error with parameters specified in Fig. 4.15......................................................116

4.17 A measured $A(\omega)$ spectrum from a 4-planet planetary gear set

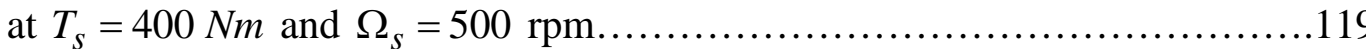

4.18 Predicted $A(\omega)$ spectrum for the same gear set as in Fig. 4.17 with AM only......

4.19 Predicted $A(\omega)$ spectrum for the same gear set as in Fig. 4.17 with FM only

4.20 Predicted $A(\omega)$ spectrum for the same gear set as in Fig. 4.17 with both AM and FM.

5.1 Measured broadband acceleration spectrum for a three planet gear set using a thin ring gear $(\Gamma=0.0025)$ at $T_{s}=500 \mathrm{Nm}$ and

(a) $\Omega_{s}=400 \mathrm{rpm}$, (b) $\Omega_{s}=442 \mathrm{rpm}$, (c) $\Omega_{s}=553 \mathrm{rpm}$, (d) $\Omega_{s}=737 \mathrm{rpm}$, (e) $\Omega_{s}=1106 \mathrm{rpm}$, and (f) $\Omega_{s}=2213 \mathrm{rpm}$ 136 


\section{CHAPTER 1}

\section{INTRODUCTION}

\subsection{Background}

Planetary gear sets, also known as epicyclic gear sets, are commonly used in many automotive, aerospace, industrial and wind turbine gearbox applications. As they employ $N$ number of identical planet branches, they allow the power transmitted to be split into $N$ parallel paths to achieve maximum power density (power to weight ratio). In Figure 1.1, a schematic of a planetary gear set with four planet gears (pinions), a sun gear, a ring (internal) gear and a planet carrier (a relatively rigid structure that supports the planets) is illustrated. Their axi-symmetric configuration requires very little radial bearing support since the radial gear mesh forces cancel out [1.1]. They are also typically quieter than their fixed-center, lay-shaft counterparts as the self-centering capability of central members (sun gear, ring gear and the carrier) not only allow wider manufacturing tolerances for the gears and the carrier [1.2], but also result in reduced noise and vibration levels [1.3]. In addition, different input-to-output speed (torque) ratios can be achieved with the same planetary gear set by simply changing the input, output and reaction (fixed) members. This feature makes them especially desirable for several applications such as automotive automatic transmissions. 


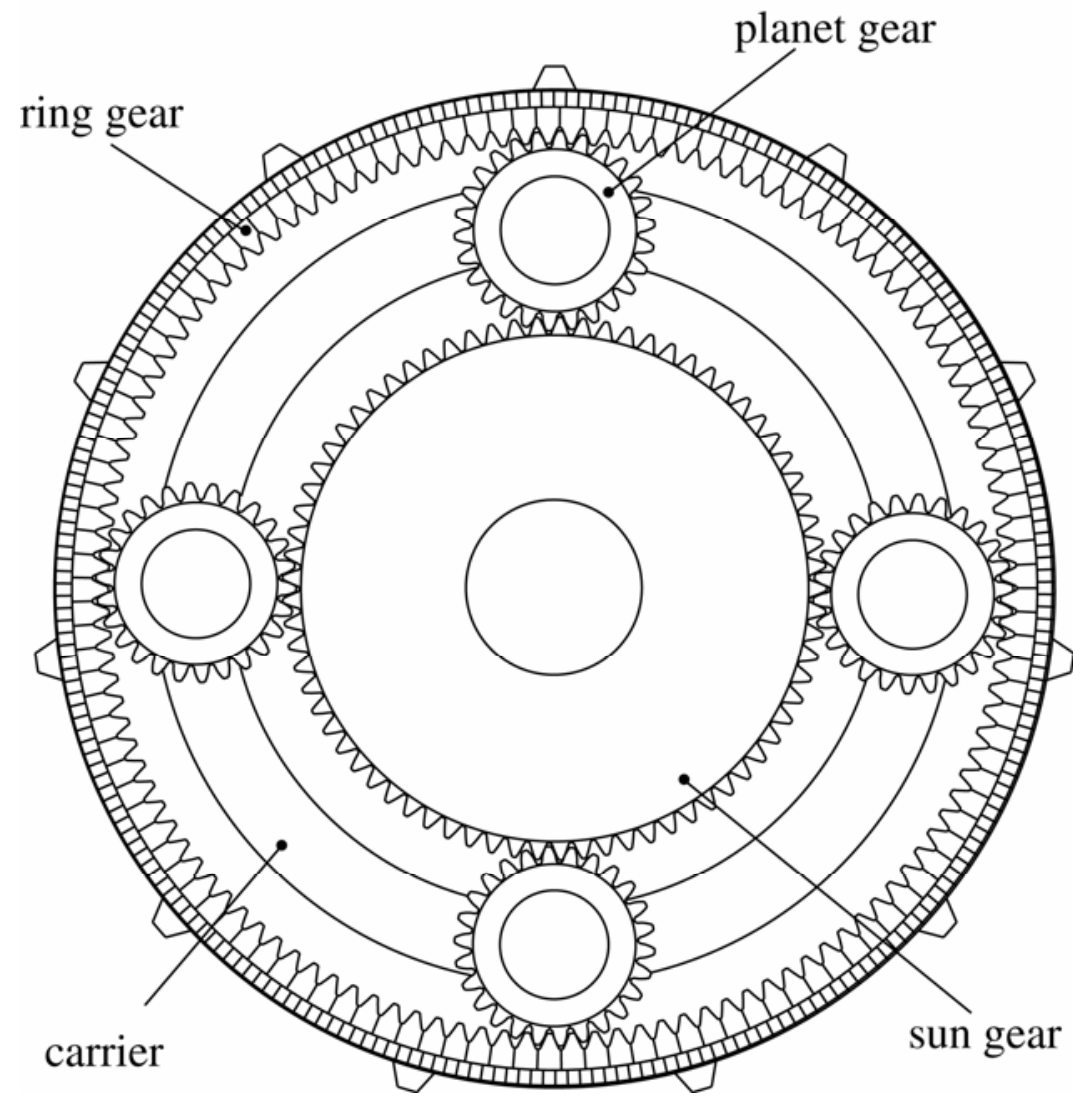

Figure 1.1: Schematic of a 4-planet planetary gear set showing its main components: a sun gear, a ring gear, four identical planet gears and a planet carrier. 
With these proven advantages in place, planetary gear sets are also known to exhibit several unique behaviors, which cannot be found in other fixed-center gear trains. Some of these behavior such as planet load sharing as a function of manufacturing errors $[1.2,1.4]$ and planet mesh phasing for cancellation or neutralization of the gear mesh excitations $[1.5,1.6]$ have been studied extensively. On the other hand, another unique behavior that most measured vibration and noise data from planetary gear transmissions exhibit, namely modulation sidebands, have not been studied in a general and systematic way. They can be recognized as harmonic orders other than the pure tones defined by the gear mesh harmonics in vibration and noise spectra of geared systems. This study focuses on a detailed investigation of the mechanisms of these sideband harmonics that appear in the vicinity of the gear mesh harmonic orders at the gear mesh frequency and its integer multiples.

\subsection{Motivation}

In spite of the fact that they constitute the most commonly used and highest volume gear set configuration with very diverse applications ranging from automotive and heavy truck automatic transmissions to rotorcraft drive trains and aerospace turbo fan gearboxes as well as in relatively new emerging application areas such as wind turbine gearboxes, there is little known about them, especially in terms of their noise and vibration behavior. This is particularly true when it comes to time-varying force transfer paths between the gear mesh of a planetary gear set and its surrounding structures. In addition, there are a very limited number of published experiments on planetary gear sets [1.7-1.10], not sufficient to bring an empirical understanding to the problem. However, 
these limited measurements and other theoretical studies, e.g. Refs. [1.9, 1.11, 1.12], were successful in pointing to a complex, yet well structured and systematic, vibration response (detailed surveys of relevant literature will be provided in individual chapters). Frequency content of measured signals was very rich with many additional sideband components located around the gear mesh harmonics in a certain order. These studies established the fact that additional peaks are due to some modulation phenomena, while shedding little light to possible mechanisms leading to these sidebands and falling short of providing general predictive capabilities to describe components of a measured planetary gear set vibration or noise spectrum. This lack of understanding of modulation sidebands of planetary gear sets forms the main motivation for this study.

Accordingly, the research undertaken in this study is primarily concerned with the modulation phenomena observed in planetary gear sets. The main causes and the mechanisms leading to modulation sidebands will be investigated both theoretically and experimentally. The emphasis will be kept on (i) bringing a fundamental understanding to the modulation sideband phenomena via simplified lower-order analytical models, (ii) demonstrating the same behavior in tightly controlled laboratory experiments, and (iii) validating the analytical models through comparisons to experiments. With these proposed contributions, this study is hoped to provide a better fundamental understanding of the vibro-acoustic behavior of the planetary gear sets as well as planetary transmissions and gearboxes. 


\subsection{Thesis Objectives and Scope}

With the overall goal of describing sources causing sidebands as well as the mechanisms they utilize, the following can be listed as the main technical objectives of this study.

(i) Develop a general analytical model to describe amplitude and frequency modulations of planetary gear sets under both quasi-static and dynamic conditions.

(ii) Using the proposed models, identify the factors and mechanisms that are responsible for modulation sidebands.

(iii) Identify key planetary gear set design parameters that define the modulation sideband behavior. Provide a physics-based classification of planetary gear sets (through these key design parameters) based on their sideband behavior.

(iv) Develop and execute an experimental test program to generate an experimental database of modulation sidebands of planetary gear sets from different classifications. Demonstrate the validity of analytical models through comparison to the data from these tightly-controlled experiments.

As the focus of the proposed research is to describe planetary gear modulation sidebands in a general and simplified way, any computational modeling techniques such as finite elements are kept outside the scope of this study. Besides required simplicity and computational efficiency, this was also a necessity since such deformable-body 
models of gear sets and geared transmissions cannot be exercised under dynamic conditions due to computational limitations. Likewise, the structural force and vibration transfer paths between gear meshes that move with the planet carrier and any fixed sensor location around the gear set will be modeled qualitatively. As there are a variety of different methods used to hold a planetary gear set in transmission housing, it is not realistic to come up with a general model that can include all these variations. Each variation indeed requires a finite elements type analysis to define force transmission through splined, bolted, pressed or serrated interfaces, which is beyond the scope of this study.

While this study is expected to provide the understanding required to relate various errors and faults (failures) to the resultant vibration or noise spectra from the planetary gear set, any prognostics or diagnostics aspects of the problems will not be included in this dissertation. While planetary gear set diagnostics have the potential to benefit from this study extensively and this study will include many factors used in gear diagnostics such as planet load sharing, this will be left to future work. Likewise, no design guidelines will be included in this study regarding which sideband structure is preferable in terms of its sound quality. All of the experiments will be limited to vibration (acceleration) measurements under deterministic conditions and subjective sound quality aspects of the problem will not be addressed here. 


\subsection{Thesis Outline}

The rest of this dissertation is arranged in four chapters. Each chapter is kept as self-sufficient as possible in terms of literature relative to the subject covered as well as figures and tables. Experimental study is presented first in Chapter 2 to help define the problem as well as providing guidance to the modeling effort presented in later chapters. First, experimental set-up and test methodology devised in these experiments are described. Details regarding the instrumentation and test planetary gear set specimens are provided together with the test matrix. Data collection, analysis and post-processing methods are also described in detail. Chapter 2 concludes with picture book of measured planetary gear set vibration spectra from various gear sets, which reveal numerous sidebands, framing the problem for the modeling studies.

In Chapter 3, a simplified mathematical model is proposed to describe the modulation sidebands of planetary gear sets due to a rotating carrier, with the assumption that the periodic dynamic gear mesh forces are known. The model includes key system parameters such as number of planets, planet position angles, and planet phasing relationships defined by the position angles and the number of teeth of the gears. The model is used to simulate a wide range of gear sets to show that they can be classified in several distinct groups based on their sideband behavior in terms of their frequencies and amplitudes. Experimental data collected in Chapter 2 for planetary gear sets from three

of these groups are used to demonstrate the capability of the analytical model of describing modulation sidebands due to rotation of the carrier. At the end, based on 
results of the parametric studies and experiments, general rules on modulation sidebands of planetary gear sets due to a rotating carrier are proposed.

In Chapter 4, a non-linear time-varying dynamic model is proposed to predict modulation sidebands of planetary gear sets with certain types of manufacturing errors operating under dynamic conditions. The discrete dynamic model takes the periodically time-varying gear mesh stiffnesses and non-linearities associated with tooth separations into account. The model uses forms of amplitude- and frequency-modulated gear mesh excitations caused by a class of gear manufacturing errors to predict dynamic forces at all gear meshes. This model is further combined with the modulation model presented in Chapter 3 to account for amplitude modulations due to the rotation of the carrier to predict acceleration spectrum at a fixed position in the planetary transmission housing. Individual contributions of each gear error in the form of amplitude and frequency modulations are illustrated through an example analysis. Comparisons are made to measured spectra from experimental database introduced in Chapter 2 to demonstrate the capability of the model in predicting the sidebands of a planetary gear set with gear manufacturing errors and a rotating carrier.

Chapter 5 provides a brief summary of the thesis, and lists major conclusions and contributions of this work to the state-of-the-art in the field of gear dynamics. At the end, recommendations are provided for future direction, including potential improvements to the model as well as applications to other related problem. 


\section{References for Chapter 1:}

[1.1] W. H. Muller, Epicyclic Drive Trains, Wayne State University Press, Detroit, 1982.

[1.2] H. Ligata, A. Kahraman, A. Singh, An experimental study of the influence of manufacturing errors on the planetary gear stresses and planet load sharing, ASME Journal of Mechanical Design, 130 (4) (2008) 041701-1 - 041701-9.

[1.3] P. Lynwander, Gear Drive Systems, Marcel Dekker Inc., New York, 1983.

[1.4] A. Bodas, A. Kahraman, Influence of carrier and gear manufacturing errors on the static load sharing behavior of planetary gear sets, JSME International Journal, Series C, 47-3 (2004) 908-915.

[1.5] A. Kahraman, G. W. Blankenship, Planet mesh phasing in epicyclic gear sets, Proceedings of International Gearing Conference, Newcastle UK, September 1994, pp. 99-104.

[1.6] A. Kahraman, Planetary gear train dynamics, ASME Journal of Mechanical Design 116 (1994) 713-720.

[1.7] M. Botman, Vibration measurements on planetary gears of aircraft turbine engines, Journal of Aircraft, 17 (5) (1980) 79 - 7012R.

[1.8] T. Hidaka, Y. Terauchi, K. Ishioka, Dynamic behavior of planetary gear $\left(2^{\text {nd }}\right.$ report: displacement of sun gear and ring gear), Bulletin of the JSME, 19-138 (1976) 1563-1570. 
[1.9] P. D. McFadden and J. D. Smith, An explanation for the asymmetry of the modulation sidebands about the tooth meshing frequency in epicyclic gear vibration, Proceedings of the Institution of Mechanical Engineers 199 C1 (1985) 65-70.

[1.10] M. Mosher, Understanding vibration spectra of planetary gear systems for fault detection, Proceedings of ASME Design Engineering Technical Conferences, Illinois USA, September 2003, Paper No: PTG-48082.

[1.11] A. L. Gu, R. H. Badgley, T. Chiang, Planet-pass-induced vibration in planetary reduction gears, ASME Design Engineering Technical Conference, New York NY USA, October 1974, Paper No: 74-DET-93.

[1.12] A. L. Gu, R. H. Badgley, Prediction of vibration sidebands in gear meshes, ASME Design Engineering Technical Conference, New York NY USA, October 1974, Paper No: 74-DET-95. 


\section{CHAPTER 2}

\section{PLANETARY GEAR SET MODULATION SIDEBAND EXPERIMENTS}

\subsection{Introduction}

In this chapter, an experimental methodology specifically developed to investigate modulation sidebands of planetary gear sets will be introduced. Among others, there were two primary goals of these experiments that were carried out in a laboratory environment. One goal was to generate an experimental database of modulation sidebands of various planetary gear sets having different configurations. This database is intended to motivate and justify the modeling studies presented in Chapters 3 and 4 as well as providing means to validate the model predictions. The other primary goal was to investigate the influence of several system-level factors such as unequal planet load sharing and ring gear deflections on the sideband behavior. In this first database where conditions are ideal (i.e. planet load sharing is equal and ring gear deflections are not excessive) will be used extensively while the second group of data will be retained for future work.

This chapter describes the experimental set-up, test methodology, planetary gear set specimens, test matrix as well as test procedures. It provides the details of the planetary gear set measurements, data collection and the data analysis. At the end, 
various measured vibration spectra are presented to demonstrate different sideband behaviors to help frame the problem in hand for the modeling effort.

\subsection{Experimental Set-up}

A set of tightly controlled planetary gear set experiments were performed for the experimental investigation of planetary modulation sidebands. An experimental set-up from a recent study on planet load sharing $[2.1,2.2]$ was used with some modifications to study the sidebands of planetary gear sets. Details of the test set-up can be found in Ref. $[2.1,2.2]$. Here, only the aspects that are directly related to this study are provided.

The test set-up uses a "back-to-back" power circulation type arrangement whose cross-section is shown in Figure 2.1(a) [2.1]. In this arrangement, the sun gears of two identical planetary gear sets (a test gear set and a reaction gear set) are connected to each other through splines on a common shaft. Likewise, the carrier of the test gear set is connected to the carrier of the reaction gear set via a rigid hollow shaft. The ring gear of the test gear set is held stationary while an external torque is applied mechanically to the ring gear of the reaction gear set forming a closed power loop. In this fixed-ring configuration, a sun gear torque $T_{S}$ is achieved conveniently by applying a torque of $T_{r}=Z_{r} T_{S} / Z_{S}$ to the reaction ring gear via a torque arm and calibrated weights, where $Z_{s}$ and $Z_{r}$ are the number of teeth for the sun gear and the ring gear, respectively. Figure 2.1(b) shows a picture of the fixtures mounted on a dynamometer. 
(a)

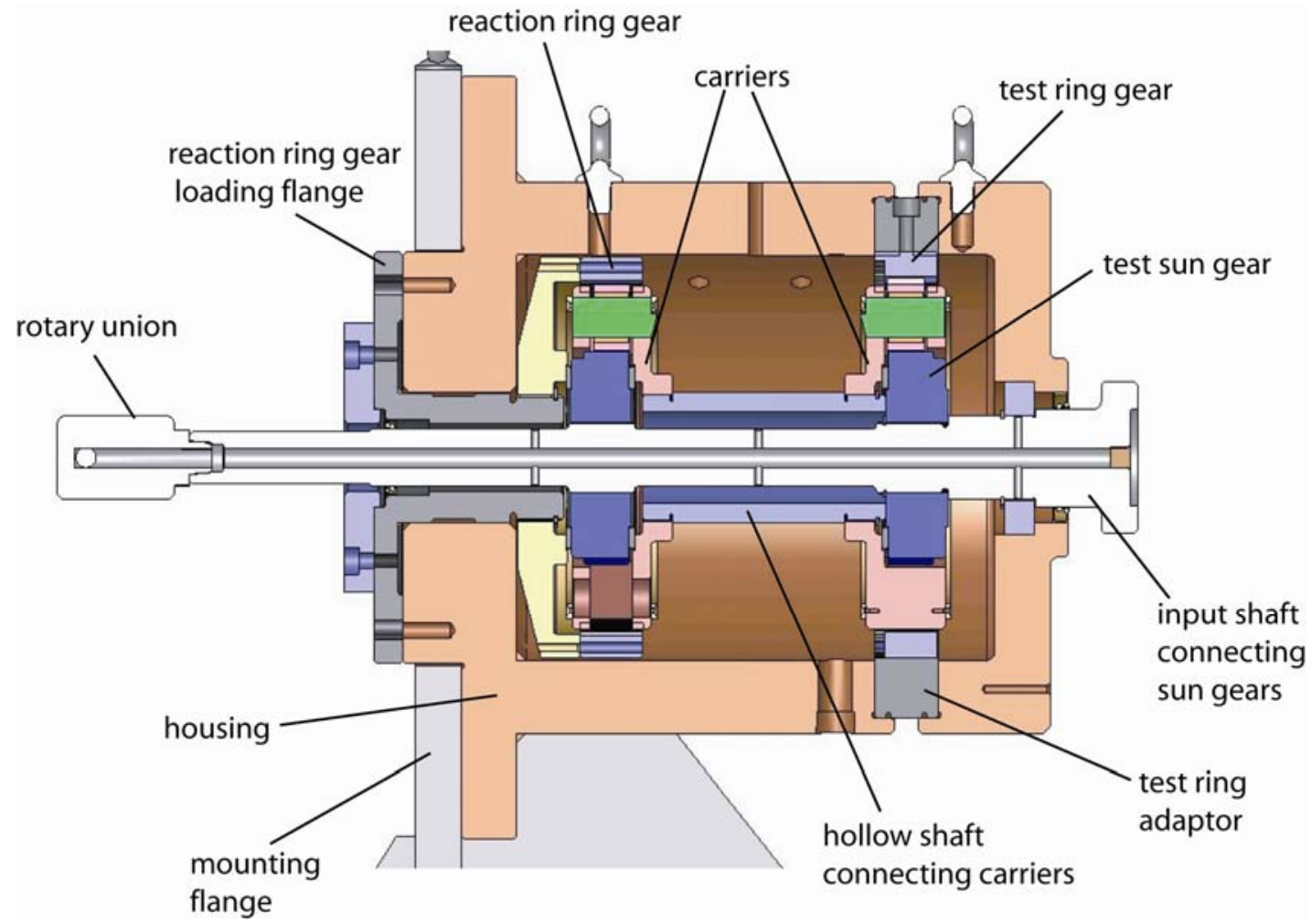

(b)

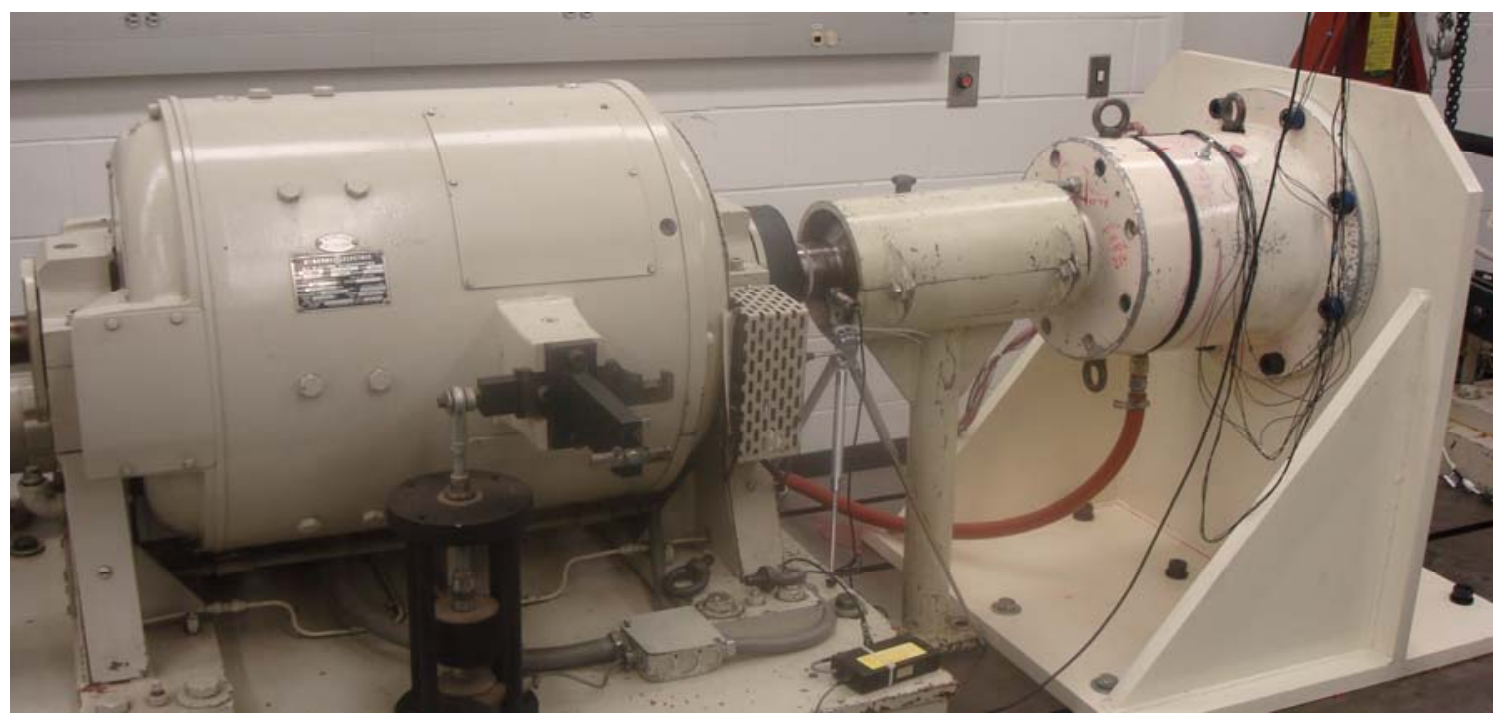

Figure 2.1: (a) Cross-section of the back-to-back planetary test fixtures, and (b) a view of the test set-up on a transmission dynamometer [2.1]. 
Here, a DC motor is connected to the shaft of the sun gears to rotate both gear sets while the gear meshes carry the load imposed by the torque trapped in the closed loop through the external torque $T_{r}$ applied on the reaction ring gear.

\subsection{Test Gear Sets and Test Matrix}

A medium-size planetary gear set design used earlier in Ref. [2.1, 2.2] was adapted here as the basic design for the test planetary gear sets. This design is based on a particular planetary gear set in an actual mid-size truck and bus automatic transmission. All of the gears shown in Figure 2.2(a) were hard ground to tight tolerances in order to achieve very accurate profiles while minimizing gear errors associated with tooth thickness and pitch line run-out in an attempt to control the once-per-revolution errors. The same level of accuracy was applied to the fabrication of the carriers shown in Figure 2.2(b) as well, so that an ideal (equal) planet-to-planet load sharing can be achieved [2.1].

The test ring gear shown in Figure 2.2(a) had a wall thickness of $10.4 \mathrm{~mm}$ that is the difference between outside radius and the root circle radius, $R_{\text {out }}-R_{\text {root }}$. With this, the ring gear thickness parameter is calculated as $\Gamma=\left(R_{\text {out }}-R_{\text {root }}\right) / R_{\text {root }}=0.083$, that was shown to exhibit modest levels of hoop deflections under load values up to $T_{S}=1,000 \mathrm{Nm}[2.3]$. The baseline gear set design has gears with $Z_{S}=73, Z_{p}=26$ and $Z_{r}=125$, where $Z_{p}$ is the number of teeth of planet gears. In this arrangement with a stationary ring gear, a 2.712 to 1 gear ratio is achieved between the input (sun gear) and the output (carrier). The test matrix included six different carriers. 
(a)

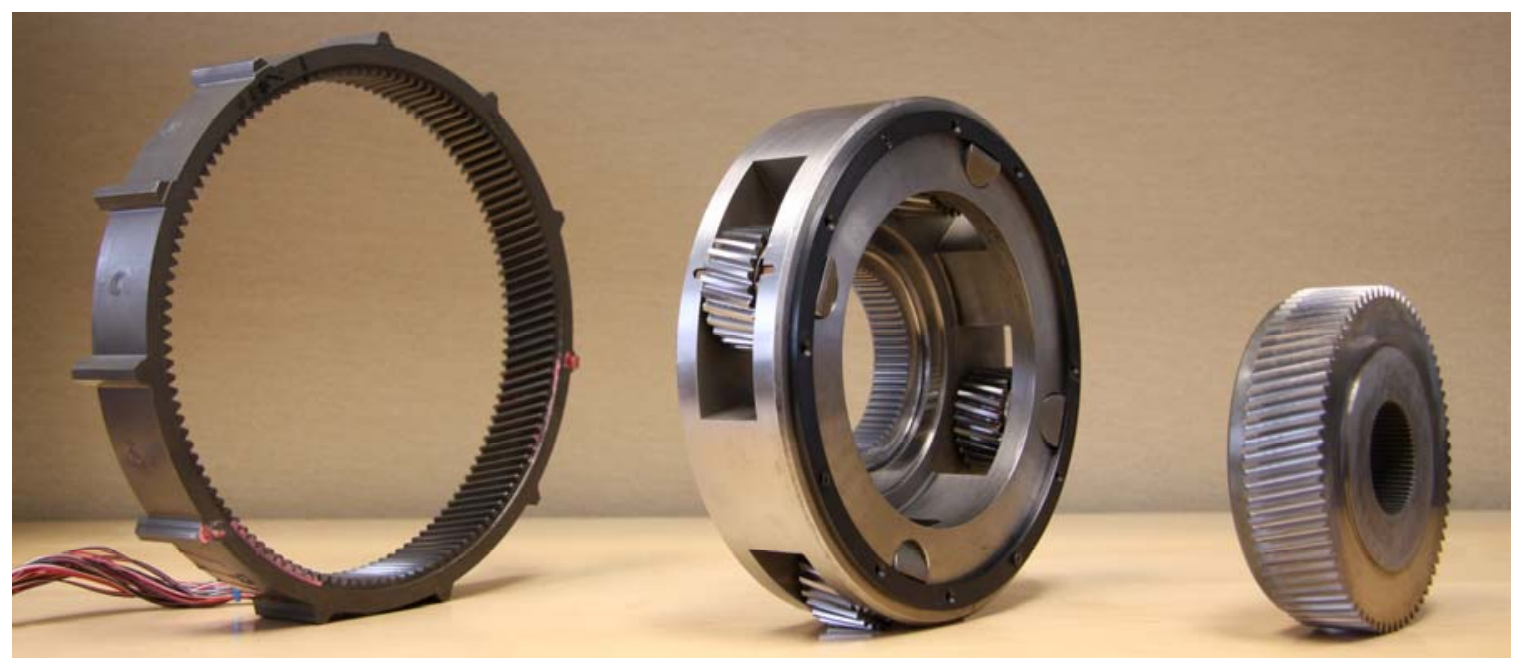

(b)

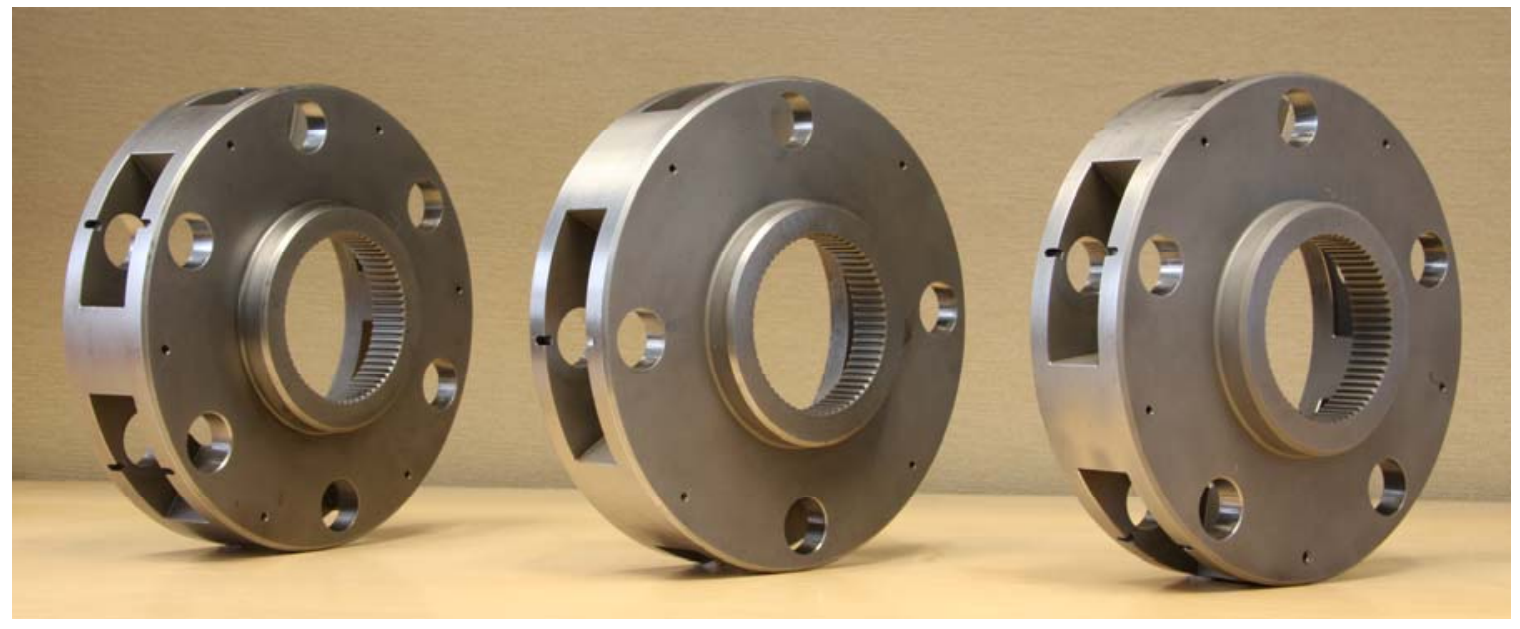

Figure 2.2: (a) A set of test gears and (b) 3, 4 and 5 planet carriers used in this study. 
Carriers with 3, 4 and 5 planets with or without intentional planet pin position errors were used (Fig. 2.2(b)). The carriers with errors were modified such that one planet pin hole was displaced from its natural position by $70 \mu \mathrm{m}$ tangentially. The quality levels of gears were such that no tangible manufacturing errors were allowed, except small amounts of run-out (eccentricity) on planet gears, which were anticipated to contribute to the measured sideband spectra.

Various tests for different gear sets and configurations were performed within the sun gear speed and torque ranges of $\Omega_{S}=200-3200 \mathrm{rpm}$ and $T_{S}=200-1000 \mathrm{Nm}$ (corresponding to a reaction ring gear torque range of $T_{r}=342-1712 \mathrm{Nm}$ ), respectively. Several of these tests were repeated more than once to ensure that the repeatability of the data is maintained throughout the duration of the entire test program.

\subsection{Instrumentation, Data Collection and Data Analysis}

The ring gear of the test side gear set was held stationary through 11 equallyspaced external spline teeth as shown in Figure 2.3. Eleven miniature accelerometers (PCB-353B15) were mounted on the outside surface of the ring gear radially at nearly equal spacing of $32.7^{\circ}$. Each accelerometer was mounted at a location representing the mid-point between the adjacent spline teeth. While only a few of these transducers would be sufficient to capture the sideband behavior, all 11 transducers were turned on through the tests in anticipation that they could be used for future ring gear vibration and force transmissibility studies. The accelerometer signals were conditioned before being recorded by a 16-channel high speed digital data recorder (SONY SIR-1000). 


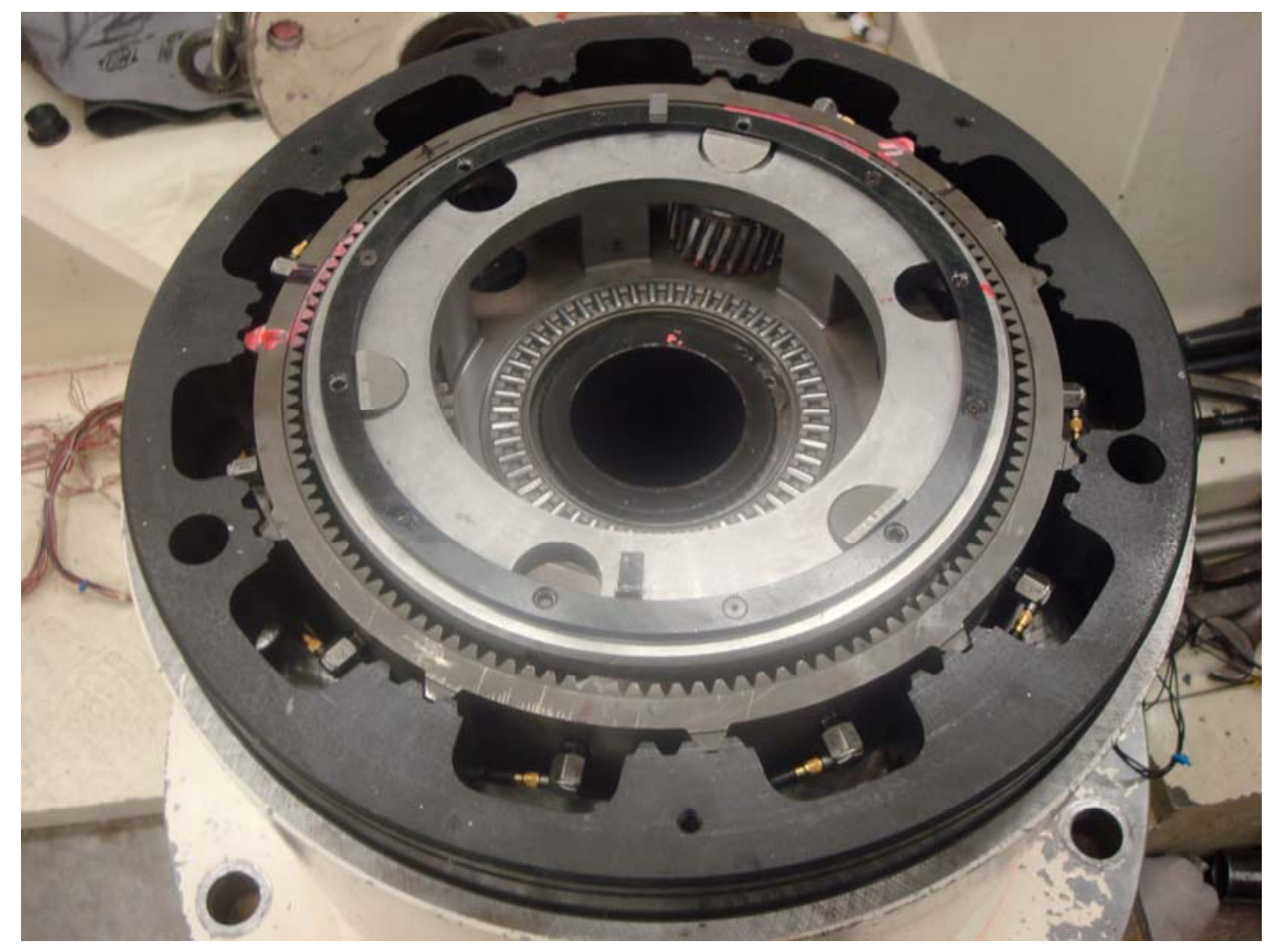

Figure 2.3: A view of the accelerometers mounted radially on the outside surface of the test ring gear. 
The data sampling rate was kept at $48,000 \mathrm{~Hz}$. The data was processed off-line to obtain the acceleration spectra measured by all 11 transducers. The acceleration spectra measured by all accelerometers were very consistent and exhibited very similar sideband behavior. For this reason, data from only one of the accelerometers will be provided in the next section. In addition, a photoelectric sensor was used to generate a once-perrevolution signal of the sun gear shaft. Using the gear reduction ratio, this was scaled to obtain a once-per-revolution signal for the rotation of the carrier, which was analyzed simultaneously with all of the acceleration signals to allow proper averaging of the collected data.

For each particular test, 5-second segments of acceleration time histories were collected together with the sun shaft reference signal. Portion of this data that include an integer number of carrier rotations (typically 6 to 96 complete carrier rotations) were isolated by a post-processor program. They were next averaged synchronously and a Fast Fourier Transform (FFT) routine was used to obtain frequency spectra corresponding to each averaged time history. The frequency scales of the resultant spectra were normalized by the carrier rotation frequency to obtain the corresponding order spectra with order one given by $H=\omega / \omega_{c}$.

\subsection{Experimental Results}

Figure 2.4 shows the example measured acceleration time histories $a(t)$ for three different gear sets at certain load and speed conditions. In these figures, the time axis is normalized by the mesh cycle period $T_{c} / Z_{r}$ and a data segment covering a complete 

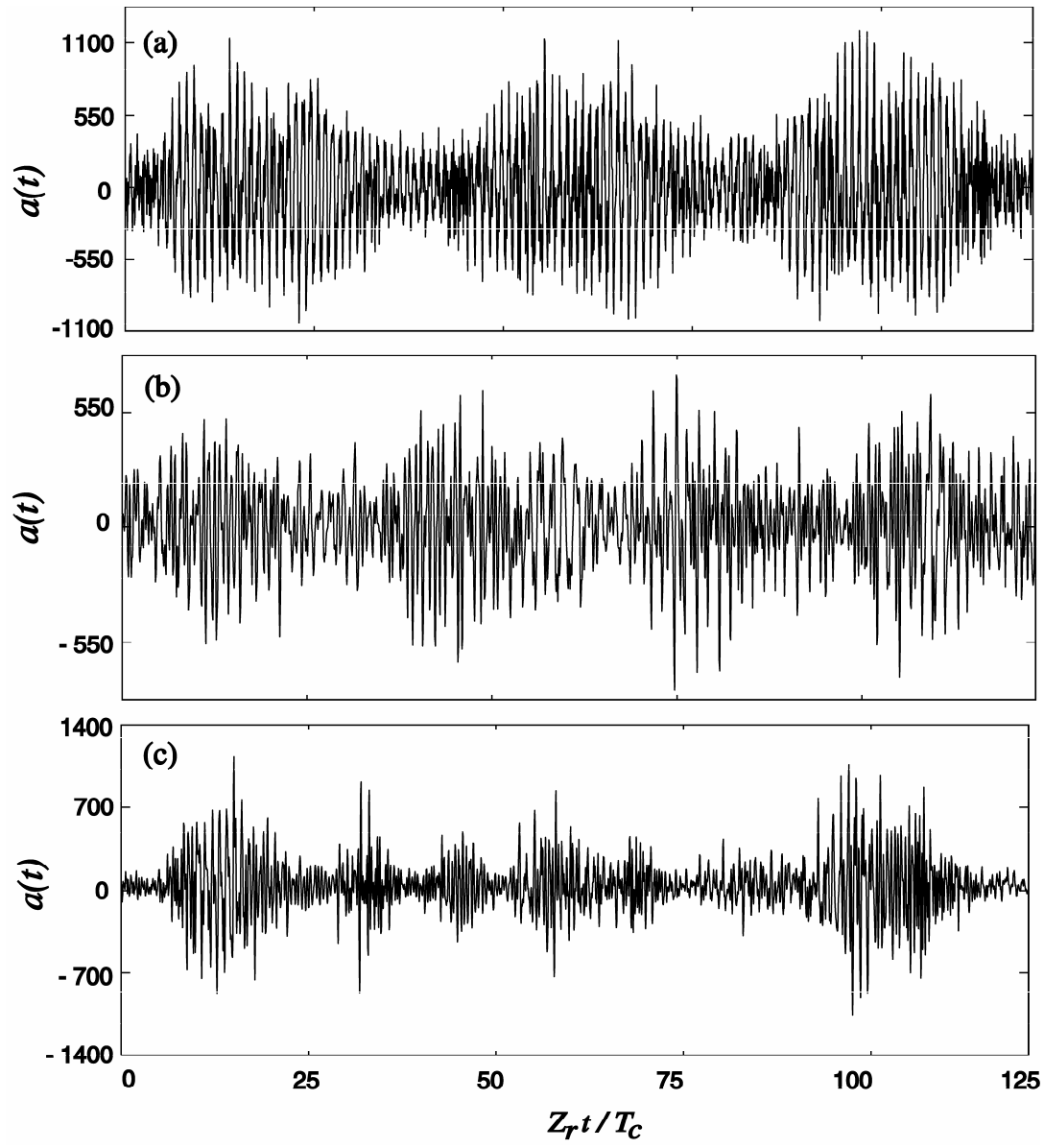

Figure 2.4: Examples of measured $a(t)$ at $\Omega_{S}=3200 \mathrm{rpm}$ from (a) a 3-planet gear set $(N=3)$ at $T_{S}=800 \mathrm{Nm},(\mathrm{b})$ a 4-planet gear set $(N=4)$ at $T_{S}=1000 \mathrm{Nm}$ and (c) a 5planet gear set $(N=5)$ at $T_{S}=1000 \mathrm{Nm}$. 
carrier rotation is presented. In Figure 2.4(a) for a gear set with three planets $(N=3)$, an apparent amplitude modulation is observed as the signal repeats itself three times. Similar modulation patterns are observed in Figures 2.4(b) and 2.4(c) for other gear sets with 4 and 5 planets, respectively. As a result, the FFT spectra $A(\omega)$ of these time histories exhibit significant number of sidebands around the fundamental gear mesh harmonic order $H_{m}$ as well as around the higher gear mesh harmonic orders $\left(2 H_{m}\right.$, $3 H_{m}$, and so on), as illustrated in the spectrum of Figure 2.5 corresponding to the time history presented in Figure 2.4(a). Here, while the amplitudes are different, the same trends are observed around each mesh harmonic order, suggesting that a careful examination of the sideband behavior around one of the mesh orders (say $H_{m}$ ) should suffice.

Additional acceleration spectra obtained for the planetary configuration with different number of planets with or without planet pin position errors are presented next, zooming in the frequency range around the fundamental gear mesh harmonic. In Figure 2.6, $A(\omega)$ for the planetary gear set with 3 equally-spaced planets is shown. This configuration does not have any planet pin position errors. This particular acceleration spectrum was obtained at $T_{S}=400 \mathrm{Nm}$ and $\Omega_{S}=500 \mathrm{rpm}$. This spectrum reveals multiple sidebands with significant amplitude and equal spacing from each other. The same planetary configuration with a different carrier was used to obtain the acceleration spectrum shown in Figure 2.7. This particular carrier had a $70 \mu \mathrm{m}$ tangential pin position error for one of the planets such that each planet carries a different amount of load. This spectrum was also obtained at $T_{S}=400 \mathrm{Nm}$ and $\Omega_{\mathrm{S}}=500 \mathrm{rpm}$. Comparison of Figures 
2.6 and 2.7 in terms of their sideband activity suggests that planet pin position errors have secondary influences on the amplitude of sidebands for the systems having 3 equallyspaced planets. This is expected since 3-planet gear sets with a radially floating sun gear (which is the case in this set-up) achieve equal load sharing even with carrier pin position errors [2.1].

Figure 2.8 shows the measured $A(\omega)$ of a planetary gear set system with $\mathrm{X}$ shaped (unequally-spaced) 4 planet configuration at $T_{S}=800 \mathrm{Nm}$ and $\Omega_{S}=500 \mathrm{rpm}$. Here, planets were positioned at $0,90.909,180$ and 270.909 degrees around the sun gear, and the carrier had no position error. A very rich sideband activity is observed here with sidebands being distributed in a qualitatively different way than the ones for 3-planet gear sets. Figure 2.9 shows a spectrum for the same gear set with a carrier having a $70 \mu \mathrm{m}$ tangential pin position error in one of its planet pin holes. This spectrum was obtained at $T_{\mathrm{S}}=600 \mathrm{Nm}$ and $\Omega_{\mathrm{s}}=500 \mathrm{rpm}$. In this case, comparison of sidebands in Figures 2.8 and 2.9 reveals differences that might be attributed to an unequal load sharing condition due to the planet pin position error applied.

An example acceleration spectrum for a gear set with 5 unequally-spaced planets (positioned at $0,72.727,143.636,216.364$ and 287.273 degrees around the sun gear) is presented in Figure 2.10 for an error-free carrier at $T_{S}=800 \mathrm{Nm}$ and an $\Omega_{S}=500 \mathrm{rpm}$. Here, again the sideband activity is very rich and somewhat complex. In addition, some qualitative differences between this spectrum and the previous ones are also observed. 


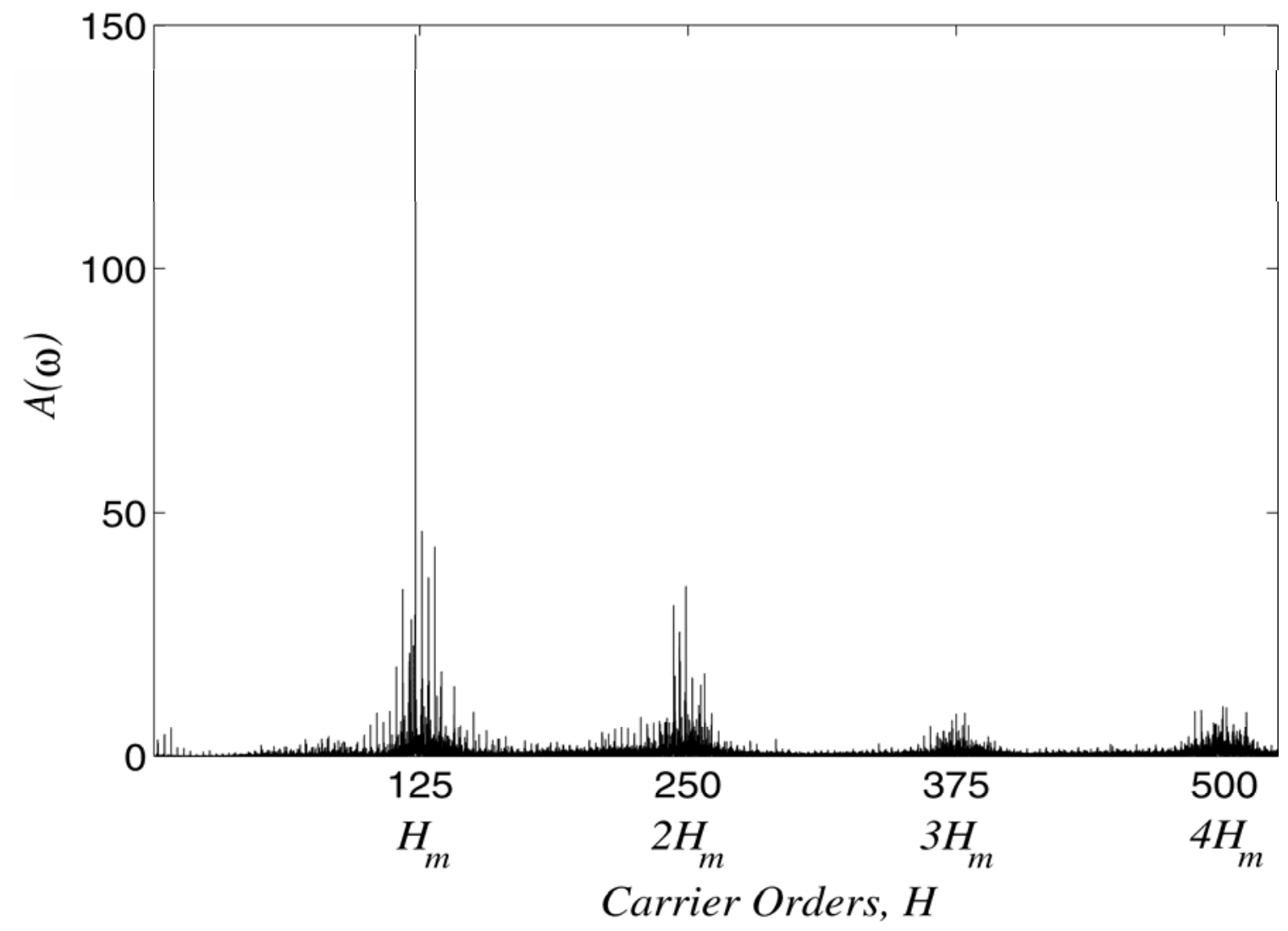

Figure 2.5: An example measured acceleration frequency spectrum from a 3-planet gear set gear set at $T_{S}=800 \mathrm{Nm}$ and $\Omega_{S}=3200 \mathrm{rpm}$. 


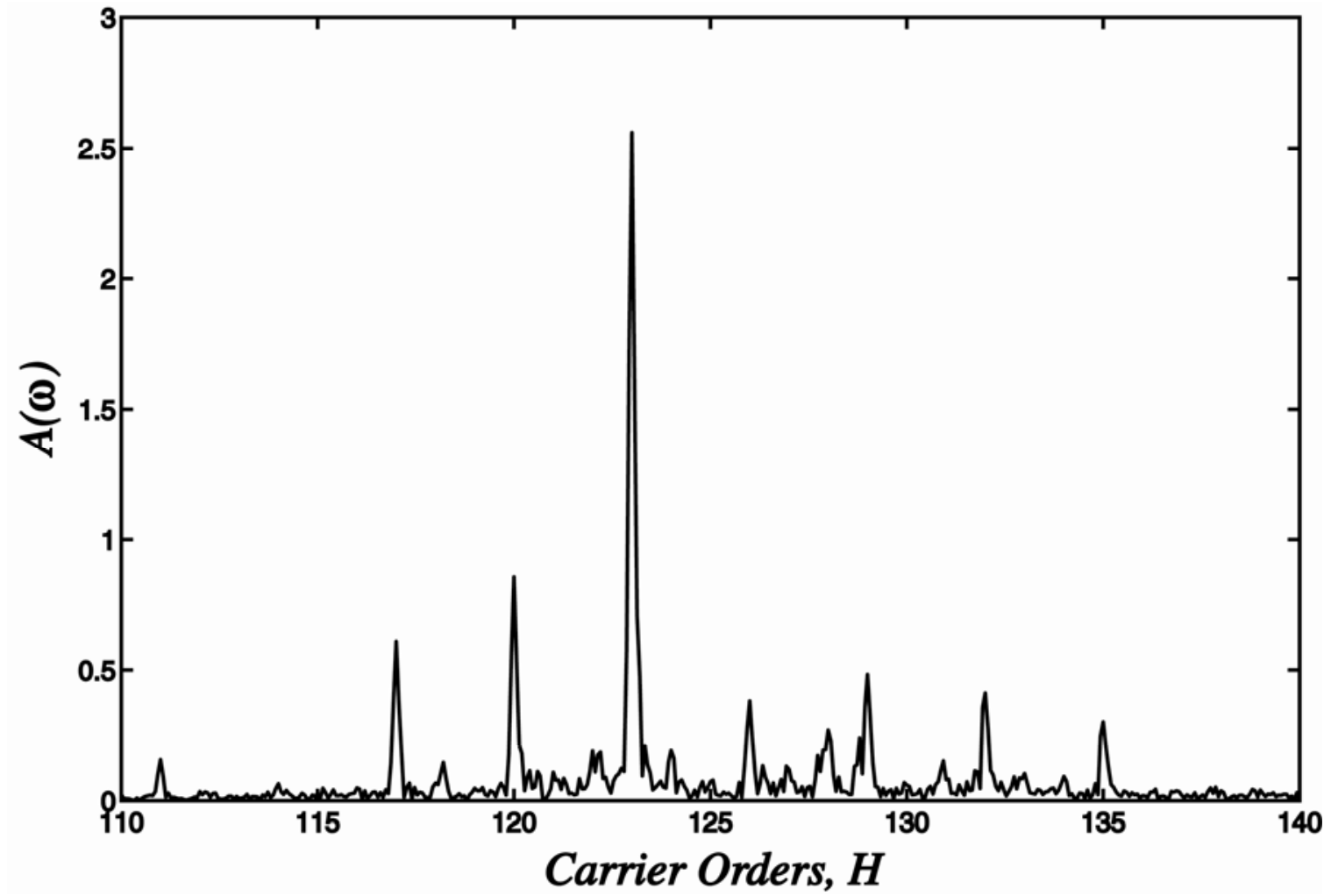

Figure 2.6: Measured acceleration spectrum from a 3-planet gear set at $T_{S}=400 \mathrm{Nm}$ and $\Omega_{S}=500 \mathrm{rpm}$. 


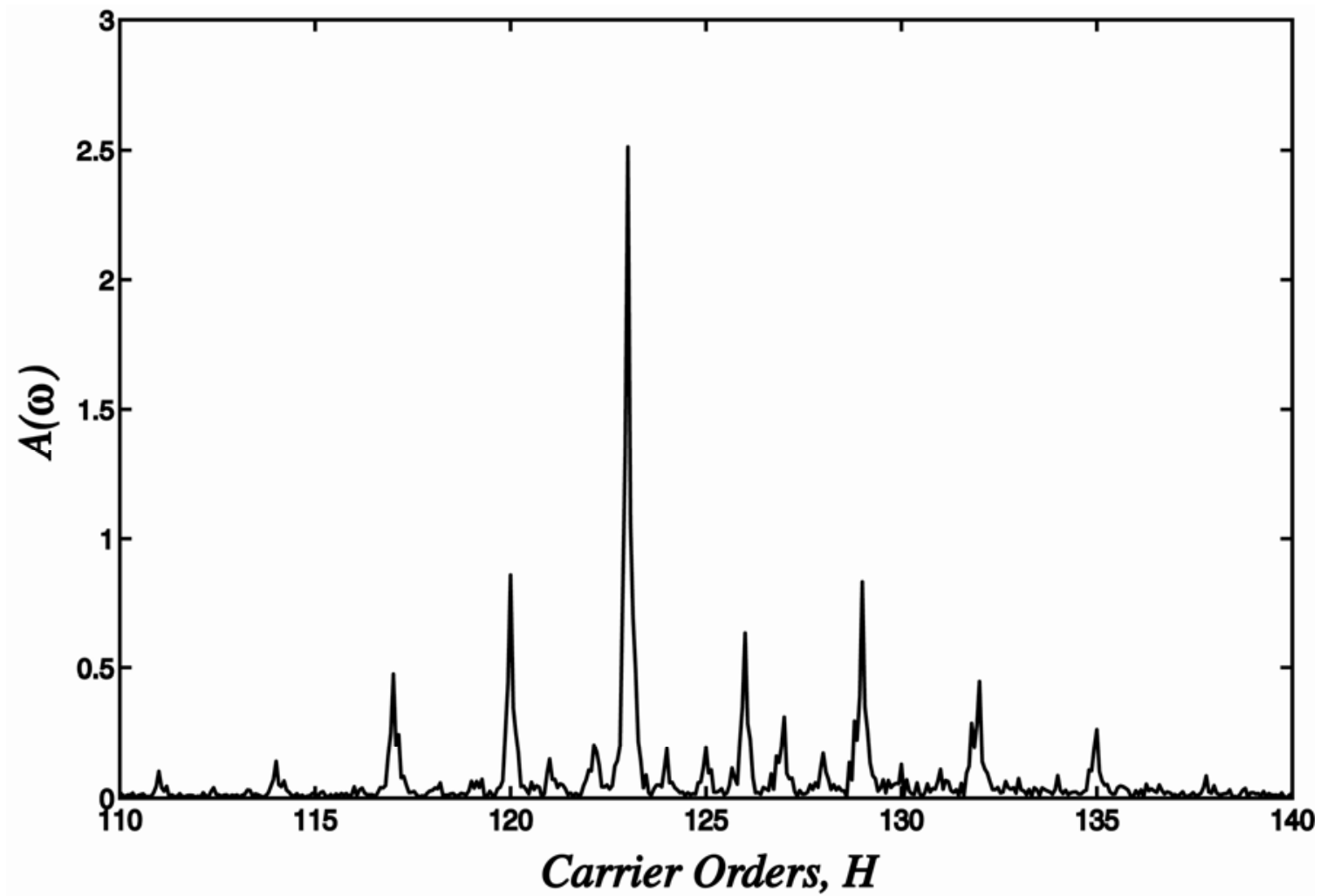

Figure 2.7: Measured acceleration spectrum from a 3-planet gear set with a planet pin position error acquired at $T_{S}=400 \mathrm{Nm}$ and $\Omega_{s}=500 \mathrm{rpm}$. 


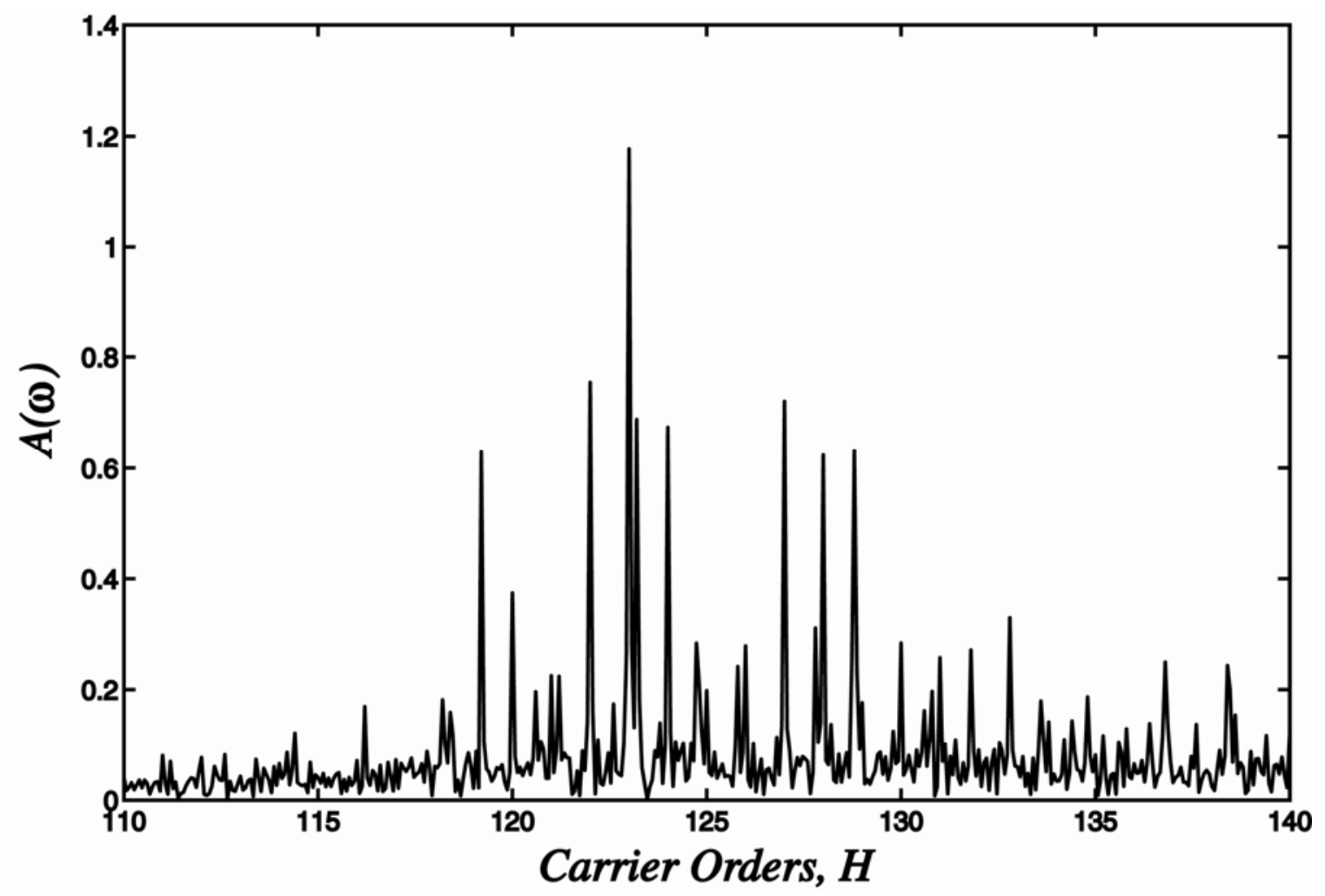

Figure 2.8: Measured acceleration spectrum from a 4-planet gear set at $T_{S}=800 \mathrm{Nm}$ and $\Omega_{s}=500 \mathrm{rpm}$. 


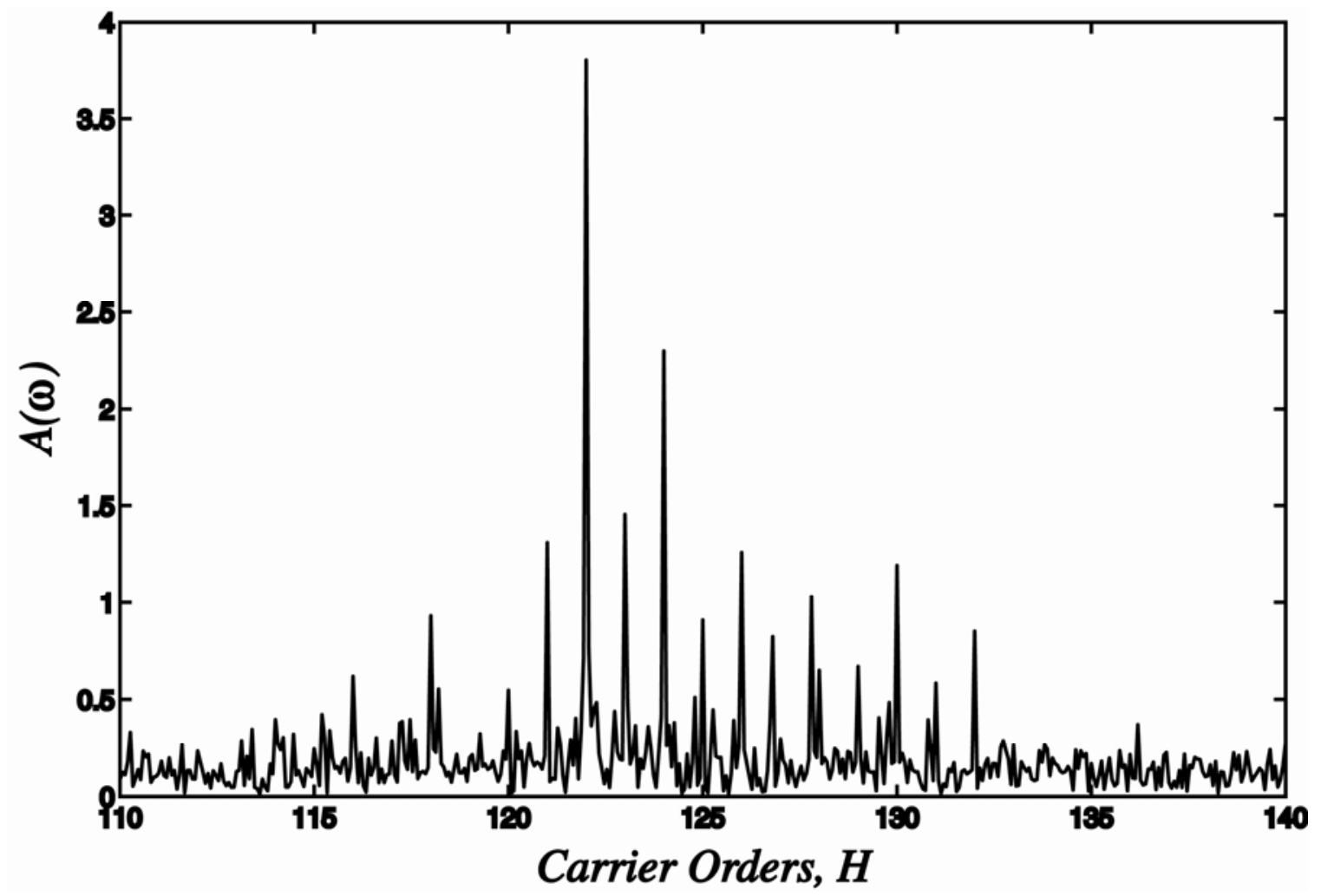

Figure 2.9: Measured acceleration spectrum from a 4-planet gear set with a planet pin position error acquired at $T_{S}=600 \mathrm{Nm}$ and $\Omega_{S}=500 \mathrm{rpm}$. 


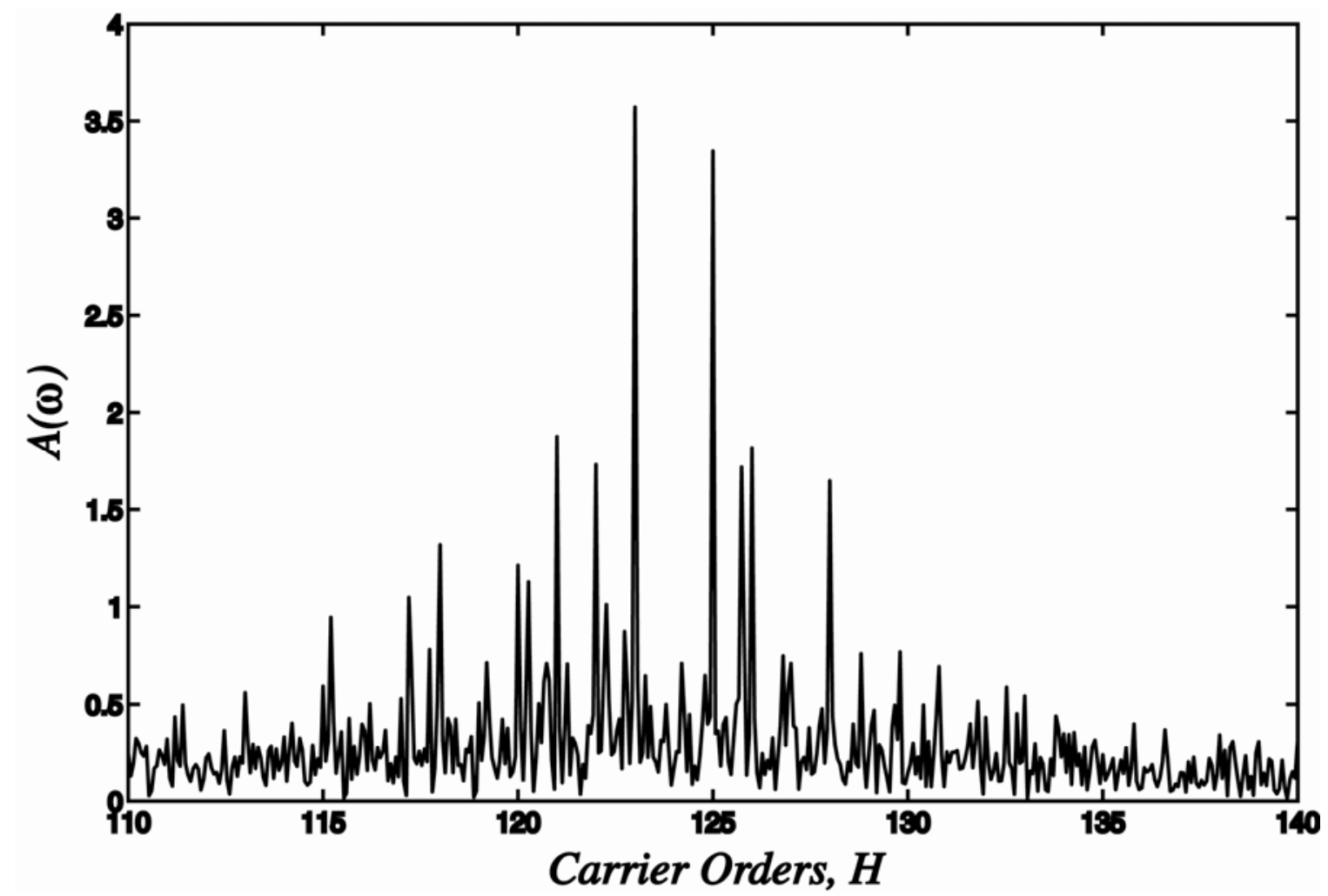

Figure 2.10: Measured acceleration spectrum from a 5-planet gear set at $T_{S}=800 \mathrm{Nm}$ and $\Omega_{s}=500 \mathrm{rpm}$. 


\subsection{Conclusions}

Measured acceleration spectra results presented in the previous section illustrate indisputably that any vibration measurement around a rotating planetary gear set contains sidebands. A closer examination of these spectra reveals the following:

(i) Some of the sidebands exist at integer orders around a gear mesh order, i.e at $H_{m} \pm n$. As $n=1$ represents the order for a complete carrier rotation, these sidebands are likely to be related to the modulations caused by a rotating carrier.

(ii) The three gear sets tested in this study result in different dominant $H_{m} \pm n$ sideband components that might occur nearly symmetric or non-symmetric around $H_{m}$. Likewise the amplitude of the fundamental gear mesh order $H_{m}$ is significant for some gear sets while it does not exist at all for others. This fact points to the potential influence of gearset parameters such as number of planets and planet position angles.

(iii) There are additional sidebands at non-integer spacing from $H_{m}$ at orders corresponding to certain gear rotation frequencies relative to the planet carrier, suggesting manufacturing errors associated with these gears might be the root cause for such sidebands.

It is clear from the data and the preliminary analysis that the planetary gear modulation sideband behavior cannot be described fully through experimental means and a general mathematical formulation is a must. Such a formulation would help identify 
the key gear set parameters and mechanisms by which sidebands formed. It would also provide the capability to design a gear set with a certain desired sideband behavior. The next two chapters will provide such an analytical treatment of this problem by including potential causes such as the carrier rotation and gear manufacturing errors. In Chapter 3, the dynamic gear mesh forces will be assumed to be known and the modulations due to carrier rotation will be described. In Chapter 4 , a discrete dynamic model of a planetary gear set will be introduced to predict dynamic gear mesh forces in presence of manufacturing errors so that the resultant sidebands due to such errors can also be determined. At the end of both Chapter 3 and Chapter 4, data collected in this chapter will be revisited to assess the accuracy of the models proposed. 


\section{References for Chapter 2:}

[2.1] H. Ligata, A. Kahraman, and A. Singh, An experimental study on the influence of manufacturing errors on the planetary gear stresses and planet load sharing, ASME Journal of Mechanical Design, 130 (4) (2008) 041701-1 - 041701-9.

[2.2] H. Ligata, Impact of system-level factors on planetary gear set behavior, Ph.D. Thesis, The Ohio State University, 2007.

[2.3] A. Singh, A. Kahraman, H. Ligata, Internal gear strains and load sharing in planetary transmissions - model and experiments, ASME Journal of Mechanical Design, 130 (7) (2008) 072602-1 - 072602-10. 


\section{CHAPTER 3}

\section{MODULATION SIDEBANDS DUE TO A ROTATING CARRIER}

\subsection{Introduction}

It was shown in measured spectra presented in Chapter 2 that at least two groups of sidebands exist in a vibration spectrum measured in near vicinity of a planetary gear set. One set of sidebands appear at integer output (carrier) orders associated with the frequency of the carrier rotation while the rest are at non-integer orders. This chapter aims at describing the sidebands occurring at integer orders through an analytical model.

Figure 3.1 illustrates a simple planetary gear set that is formed by a sun gear $(s)$, an internal or ring gear $(r)$ and $N$ number of identical planet gears (pinions) located around the sun gear at angles $\psi_{i}(i \in[1, N])$. In this example, $N=4$. The planets are held by a common rigid structure, called the planet carrier $(c)$ through planet bearings. In this arrangement, planets act as idler gears with no connections to the outside structures of the gearbox, while the central members $s, r$, and $c$ are either connected to input or output, or fixed with respect to transmission housing. In cases when the sun or ring gear is fixed (stationary), the carrier must rotate with its planets. For instance, in Figure 3.1, the ring gear is fixed through a set of outside splines to the housing, and a transducer 


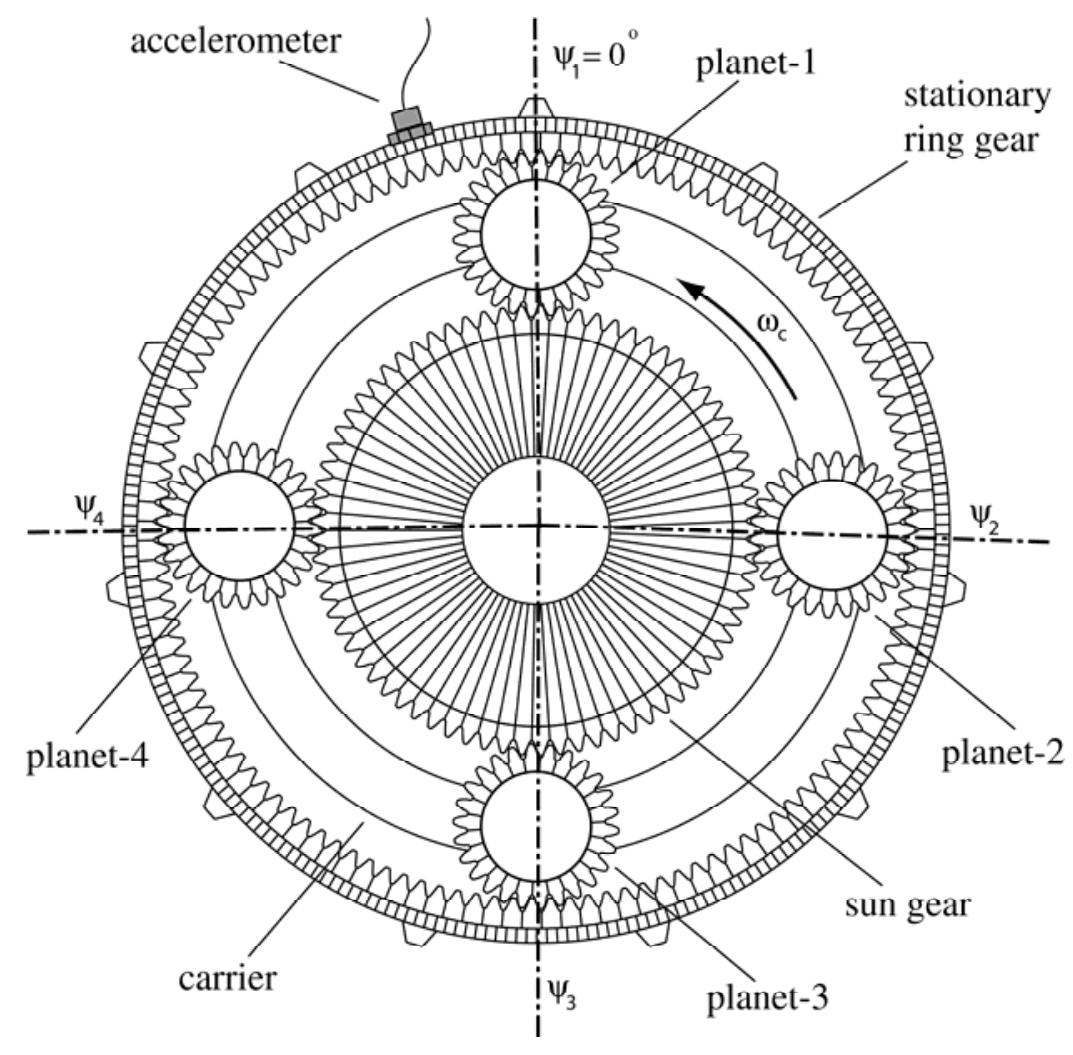

Figure 3.1: A schematic showing the components of a 4-planet planetary gear set and an accelerometer mounted on the ring gear. 
mounted on it or on the housing near the ring gear housing interface experiences a periodic variation in vibration amplitudes as planets pass through this fixed transducer location. This time variation of the location of planet meshes relative to the fixed measurement point is known to cause an amplitude modulation (AM) of vibration and noise time histories to result in modulation sidebands in the frequency domain. This is one of the main mechanisms causing planetary gear sidebands.

In addition to sidebands caused by a rotating carrier, a large number of gear manufacturing errors can also cause variations to modulate the dynamic gear mesh forces in three different forms of wave modulations, namely amplitude modulations, frequency modulations $(\mathrm{FM})$, and phase modulations (PM). These types of modulations were reported to occur frequently in mechanical systems [3.1, 3.2]. Typical errors of a planetary gear set that might cause modulations include eccentricities and run-out errors of the gears and the carrier, and tooth-to-tooth spacing, tooth thickness and indexing errors $[3.3,3.4]$. There are other variations due to relative motion of the rotating components of the gear set with respect to the other components that might result in sidebands as well. Among them, radial floating motion of central members, such as the sun gear, induces instantaneous center distance variations and modulates the gear mesh forces [3.5]. Such effects will be investigated in Chapter 4.

There are only a few published studies on planetary gear sidebands with significant contributions to the understanding of this subject matter. In one such study, McFadden and Smith [3.6] predicted the vibration produced at a fixed transducer location on the ring gear of a simple planetary gear set. Their study was the first to explain the underlying reasons for the asymmetric distribution of the modulation sidebands about the 
tooth meshing frequency. Their model was able to predict the frequency content of the dominant vibration peaks of an example planetary gear set, but not the relative amplitude content. Later, McNames [3.7] employed continuous-time Fourier series to explore the relative amplitudes of the dominant peaks that can possibly show up in the spectra along with their corresponding frequencies. His model was based on the time-domain model of McFadden and Smith [3.6], with the discrete Fourier series analysis included. McNames reported that the total vibration spectra (sum of the vibrations from each individual planet gear as they pass through a fixed transducer location) will have dominant components only at frequencies that are integer multiples of number of planets in the system. This conclusion was presented for a class of planetary gear sets (equally spaced planets with sequential planet mesh phasing) and its validity was not tested for other classes of planetary gear sets. Besides these two studies, there are a variety of works that deal with sidebands in the wider context of planetary gear vibrations [3.8-3.16]. The same is true for studies on planetary vibration condition monitoring for diagnostics purposes [3.163.20]. While providing a reasonable foundation for amplitude modulated sidebands of planetary gear sets, above studies fall short of providing a general formulation that can be used to classify planetary gear sets based on their sideband behavior and describe the sideband behavior of any arbitrary planetary gear set. Also missing from the literature, besides very limited data provided in Ref. [3.6, 3.7] from actual field tests of planetary transmissions, is a detailed and dedicated experimental study of modulation sidebands of planetary gear sets for validation of any of these models.

Accordingly, this chapter first focuses on developing a simplified analytical model to describe amplitude modulations of planetary gear sets due to a rotating carrier. 
This model is intended to be general so that any simple planetary gear set having any planet spacing condition (equally- or unequally-spaced), any number of planets, any number of gear teeth as well as any planet mesh phasing condition (in-phase, sequentially phased or arbitrarily phased) can be analyzed. The model will be used to show that there are various classes of planetary gear sets that exhibit different sideband behavior. Trends observed from each class of gear set will be documented. The second objective of this chapter is to demonstrate the validity of these trends through the experimental study presented in Chapter 2. Several measured acceleration frequency spectra will be compared to the predictions to assess the fidelity of the proposed analytical model.

\subsection{Analytical Model}

For a planetary gear set with $N$ planets, consider the kinematic configuration when the sun gear $s$ and the planet carrier $c$ act as the input and output members and the ring gear $r$ is held stationary (its rotational velocity $\omega_{r}=0$ ). In this kinematic configuration, the fundamental gear mesh (tooth passing) frequency is defined as

$$
\omega_{m}=Z_{r} \omega_{C}
$$

where $\omega_{C}$ is the absolute angular velocity of the planet carrier and $Z_{r}$ is the number of teeth of the ring gear. In case of the fixed sun gear, $Z_{r}$ is replaced by the number of teeth of the sun gear $Z_{S}$. The planets are positioned at angles $\psi_{i}(i \in[1, N])$ within the planet carrier. Setting $\psi_{1}=0$ without any loss of generality, other planets can be 
assembled in any set of discrete angles as long as they are integer multiples of the least mesh angle which can be defined as

$$
\lambda=\frac{2 \pi}{Z_{S}+Z_{r}} .
$$

Planet $i$ cannot be assembled at a position angle unless $\psi_{i}=m \lambda$ ( $m$ : integer). Further, a gear set with equally-spaced planets at planet position angles $\psi_{i}=2 \pi(i-1) / N$ is possible only if $\left(Z_{s}+Z_{r}\right) / N=$ integer. For instance, for a gear pair with $Z_{r}=125$ and $Z_{S}=73, \lambda=1.8182^{\circ}$ and a 3 -planet carrier can be assembled in equal spacing $\left(\left(Z_{S}+Z_{r}\right) / N=\frac{1}{3}(73+125)=66=\right.$ integer $)$ while 4 and 5 -planet carriers cannot be assembled in equal spacing with these gears $\left(\frac{1}{4}(73+125)=49.5\right.$ and $\left.\frac{1}{5}(73+125)=39.6\right)$

Here, each mesh of the ring gear with each planet $i$ transmits a static (mean) mesh force $\bar{F}_{i}$ as well as a dynamic force $F_{i}(t)$ about $\bar{F}_{i}$ induced by vibratory behavior of the gear pair [3.21, 3.22]. This dynamic mesh force component is periodic at the gear mesh frequency and can be written in Fourier series for the fixed ring gear case as

$$
F_{i}(t)=\sum_{j=1}^{J} F_{i j} \cos \left(j Z_{r} \omega_{c} t+\phi_{j}+j Z_{r} \psi_{i}\right)
$$


where $F_{i j}$ is the Fourier coefficient of $j$-th harmonic of the dynamic force of ring-planet $i$ mesh, $\phi_{j}$ is the phase angle of the $j$-th harmonic component and $Z_{r} \psi_{i}$ is the phase angle between the ring gear meshes of planet $i$ and planet 1 [3.21, 3.23, 3.24].

In Figure 3.1, the measured acceleration for the transducer mounted on the outer surface of the ring gear will be modulated as $F_{i}(t)(i \in[1, N])$ that applies along the line of action of the ring-planet $i$ mesh rotates with the carrier at a velocity $\omega_{c}$. For a complete revolution of the carrier, the transducer will experience the disturbances from all $N$ planets in sequence. As the force transmission path between the ring-planet $i$ mesh is rather complex (and can be described quantitatively only by dynamic deformable-body analysis), individual influence of planet-i on the transducer will be assumed to last for a duration of $T_{C} / N$ where $T_{C}=2 \pi / \omega_{C}$ is the rotational period of the carrier. With this assumption, as planet $i$ approaches to the transducer location, its influence will increase for the first $T_{C} / 2 N$ time period, reaching its maximum when planet $i$ is at the transducer location and then diminishing to zero at the end of the next $T_{C} / 2 N$ time period. This will be followed by planet $(i+1)$ that is assumed to dominate the response of the transducer for the next $T_{C} / N$ time period, and so on. A Hanning function is conveniently used here to represent this phenomenon:

$$
w(t)=\frac{1}{2}-\frac{1}{2}\left[\cos \left(\frac{2 \pi N t}{T_{C}}\right)\right] .
$$

With this, for a planet $i$ positioned at angle $\psi_{i}$, a weighting function is defined as 


$$
w_{i}(t)=W_{i} w\left(t-\frac{\psi_{i}}{2 \pi} T_{C}\right) U_{i}(t)
$$

where $U_{i}(t)$ is defined as

$$
U_{i}(t)=\sum_{n=1}^{\infty}\left\{u\left[t-\left(\frac{(n-1) N+i-1}{N}\right) T_{C}\right]-u\left[t-\left(\frac{(n-1) N+i}{N}\right) T_{C}\right]\right\}
$$

In this equation, terms $u(t-a)$ are unit step functions $(u(t-a)=1$ for $t>a$ and $u(t-a)=0$ for $t<a)$ that ensure the influence of planet $i$ on the transducer lasts only for a period of $T_{C} / N$. The summation over $n$ is needed to maintain the same periodicity for each carrier rotation. In Eq. (3.4b), the multiplier $W_{i}=N \bar{F}_{i} / \sum_{j=1}^{N} \bar{F}_{j}$ is intended to account for any unequal load sharing amongst the planets due to the carrier and gear manufacturing errors $[3.3,3.4]$. If all $\bar{F}_{i}$ are the same (i.e. perfect load sharing), then all $W_{i}=1$, in addition to having identical Fourier harmonic amplitudes $F_{i j}$ for $i \in[1, N]$ in Eq. (3.3). However, if all the $N$ planets do not carry equal share of the load, then $\bar{F}_{i}$ (and $W_{i}$ ) will be different in addition to $F_{i j}$ that must be determined at this $\bar{F}_{i}$ value [3.22].

Equations (3.4) represent major simplifications employed by the model. The assumption that the influence of planet $i$ on the transducer lasts only for a period of $T_{C} / N$ might not be true for certain systems, especially if the ring gear is very flexible. The same formulation can easily be modified to increase the duration in which a planet is effective. The weighting function $w(t)$ defined in Eq. (3.4a) was also chosen rather arbitrarily to represent the continuously increasing and then diminishing effect of a planet 
mesh on the observed vibration amplitude. A more accurate and realistic shape for $w(t)$ should be specific to each gear set considered, depending on its size, ring gear-housing interface and ring gear flexibility. A proper accounting of these effects would require a deformable-body dynamic model that can represent the transfer path between a given gear mesh and the point of measurement accurately.

The acceleration signal caused by dynamic force $F_{i}(t)$ of the ring/planet $i$ mesh is assumed to be proportional to the product of $w_{i}(t)$ and $F_{i}(t)$ so that

$$
a_{i}(t)=C w_{i}(t) F_{i}(t)=\bar{W}_{i} w\left(t-\frac{\psi_{i}}{2 \pi} T_{C}\right) U_{i}(t) \sum_{j=1}^{J} F_{i j} \cos \left(j Z_{r} \omega_{C} t+\phi_{j}+j Z_{r} \psi_{i}\right)
$$

where $\bar{W}_{i}=C W_{i}$ and $C$ is a constant accounting for the physical unit difference of the acceleration and force sides of the Eq. (3.5a). Figure 3.2 schematically illustrates $F_{i}(t)$, $w_{i}(t)$ and the total acceleration signal $a(t)$ that is given as

$$
a(t)=\sum_{i=1}^{N} a_{i}(t)
$$

for a 3-planet $(N=3)$ gear set having equal spacing $\left(\psi_{1}=0, \psi_{2}=2 \pi / 3\right.$ and $\left.\psi_{3}=4 \pi / 3\right)$, assuming harmonic $F_{i}(t)\left(J=1\right.$ in Eq. (3.5a)) with $\bar{W}_{i}=1$ and $Z_{r}=25$. Since the overall time signal $a(t)$ shown in Figure 3.2 is heavily modulated, the corresponding frequency spectrum 


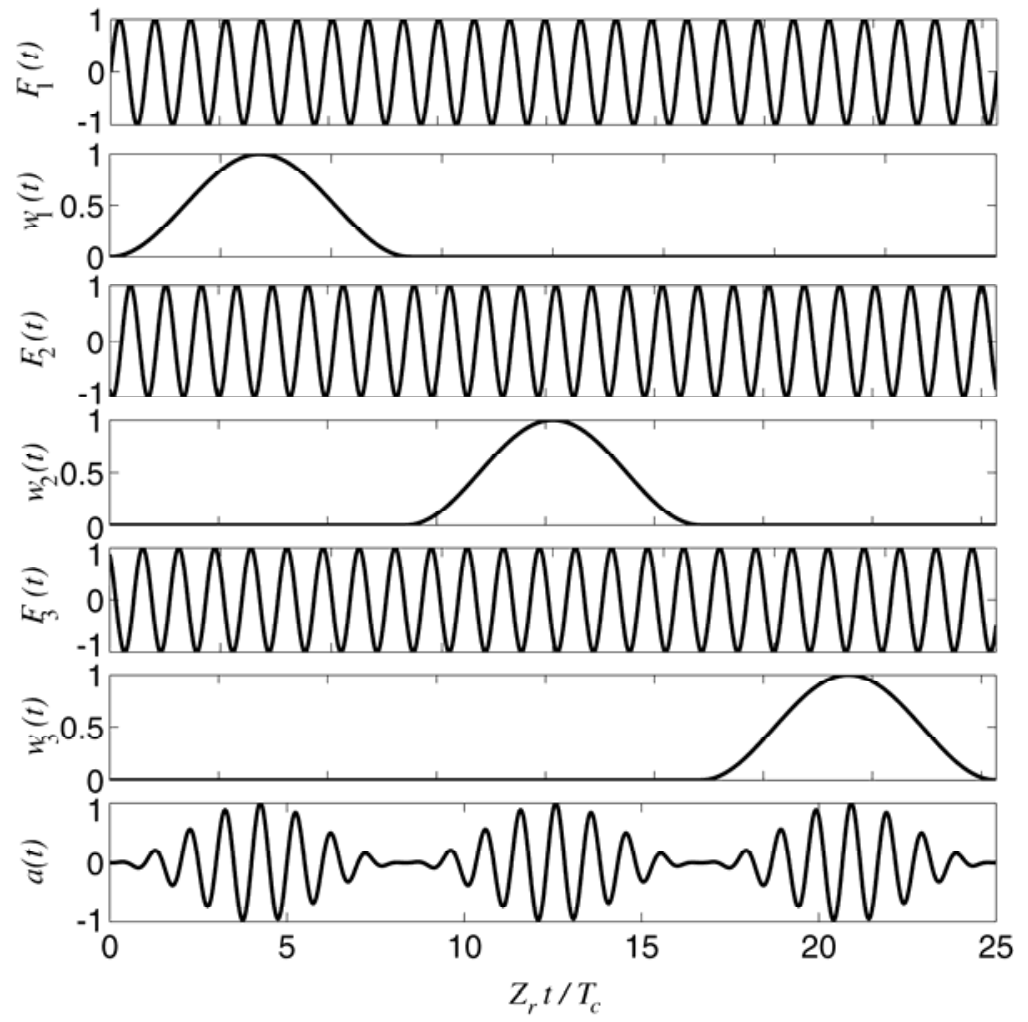

Figure 3.2: A schematic illustration of $F_{i}(t), w_{i}(t)$ and $a(t)$ for a gear set having $N=3$ and $Z_{r}=25$. 


$$
A(\omega)=\left|\int_{-\infty}^{\infty} a(t) e^{-i \omega t} d t\right|
$$

would reveal significant sideband activity around the gear mesh harmonic frequency $\omega_{m}=Z_{r} \omega_{C}$.

Equation (3.5a) should work for planetary gear sets that act in a linear timevarying fashion as it is the case for most planetary gear sets formed by helical gear sets. The time-varying coefficients are due to periodic fluctuations of the gear mesh stiffnesses that act as parametric excitations for the predicted periodic dynamic gear mesh forces $F_{i}(t)[3.22,3.25]$. In spur planetary gear sets, however, it is possible to have nonlinear behavior within the primary and parametric resonance regimes [3.25] limiting the use of Eq. (3.5a) to the operating speeds within the off-resonance regions.

In the presence of manufacturing errors that are periodic over a complete carrier (ring gear) revolution as described in Section 3.1, one would be required to define another function in the form

$$
R(\theta)=\bar{R}+\sum_{k=1}^{K} R_{k} \sin \left(k \theta+\phi_{k}\right)
$$

where, $\bar{R}$ is the average over complete rotation. Here, $k$ is the harmonic index, and $R_{k}$ and $\phi_{k}$ are the amplitude and the phase angle of that particular Fourier component. Each planet $i$ is subjected to this variation with $\theta_{i}=\omega_{c} t+\psi_{i}$ so that 


$$
R_{i}(t)=\bar{R}+\sum_{k=1}^{K} R_{k} \sin \left(k \omega_{c} t+k \psi_{i}+\phi_{k}\right) .
$$

With this additional variation,

$$
a_{i}(t)=w_{i}(t) F_{i}(t) R_{i}(t)
$$

and Eq. (3.5b) is still valid, now with this form of $a_{i}(t)$. Similar variations can be introduced at the sun gear rotational frequency to account for the errors associated with it, while they should be less important as path between each sun mesh and the transducer is longer and sun is commonly allowed to float radially to compensate for some of these errors.

\subsection{Classification of Planetary Gear Sets based on Their Sideband Behavior}

Assuming that $R_{i}(t)=\bar{R}$, Eq. (3.5) indicates that there are $N+2$ main parameters $\left(N, Z_{r}, \psi_{1}, \psi_{2}, \ldots, \psi_{N}\right)$ dictating any sideband activity observed in frequency domain. With the restrictions induced by Eq. (3.2) in terms of where planets can be positioned for suitable assembly conditions, five different conditions are possible, each causing a different form of modulation sidebands. This fact was also indicated and proven by numerous simulations that utilize the current model introduced. Specifically chosen simulation results will be presented next along with the cases they belong. 


\section{Case (i): Equally-spaced planets and in-phase gear meshes}

This condition is defined mathematically as

$$
\psi_{i}=\frac{2 \pi(i-1)}{N} \quad \text { and } \quad \frac{Z_{r} \psi_{i}}{2 \pi}=n \quad(n: \text { integer })
$$

This first condition indicates that the planets are equally spaced while the second condition ensures all $F_{i}(t)$ in Eq. (3.3) are in phase. For this case with equally-spaced planets, a simpler form of the second condition is that $Z_{r}$ must divisible by $N$ (i.e. $Z_{r} / N=$ integer ) for all ring gear meshes to be in phase. Assuming equal load sharing $\left(W_{i}=1, F_{i j}=F_{j}, i \in[1, N]\right)$ and considering a single harmonic term $(J=1)$ in Eq. (3.3) as the mesh forces that are in phase, $F_{i}(t)=F(t)=F_{1} \cos Z_{r} \omega_{c} t$, further ignoring the onceper revolution errors in Eq. (3.8), Eq. (3.5b) is written as

$$
a(t)=F(t) \sum_{i=1}^{N} w_{i}(t)=F(t) \sum_{i=1}^{N} w\left(t-\frac{\psi_{i}}{2 \pi} T_{C}\right) U_{i}(t)
$$

Under the conditions assumed in this case, $w(t)$ becomes periodic at $T_{C} / N$, reducing Eq. (3.11a) to

$$
a(t)=C F(t) w(t)=C F_{1} \cos \left(Z_{r} \omega_{C} t\right)\left[\frac{1}{2}-\frac{1}{2} \cos \left(N \omega_{C} t\right)\right]
$$

which can be written as 


$$
a(t)=C F_{1}\left\{\frac{1}{2} \cos \left(Z_{r} \omega_{C} t\right)-\frac{1}{4} \cos \left[\left(Z_{r}+N\right) \omega_{C} t\right]-\frac{1}{4} \cos \left[\left(Z_{r}-N\right) \omega_{C} t\right]\right\} .
$$

This indicates that the corresponding frequency spectrum will have gear mesh component at frequency $Z_{r} \omega_{C}$ and two symmetric sidebands at half amplitude as the mesh harmonic at frequencies $\left(Z_{r}+N\right) \omega_{C}$ and $\left(Z_{r}-N\right) \omega_{C}$.

Figure 3.3 illustrates $A(\omega)$ spectra (with $C F_{1}=1$ ) for four different planetary gear sets, satisfying conditions given in Eq. (3.10). The first gear set represented by Figure 3.3(a) has $N=3$ with $Z_{r}=123$ and $Z_{s}=72$. The second gear set in Figure 3.3(b) is a 4-planet system $(N=4)$ with $Z_{r}=124$ and $Z_{S}=72$, while a 5-planet gear set with $Z_{r}=125$ and $Z_{S}=70$ is represented by the spectrum of Figure 3.3(c). Finally, a gear set having $N=6, Z_{r}=126$ and $Z_{S}=72$ results in the spectrum shown in Figure 3.3(d). All four spectra correspond to Eq. (3.11c).

It can be concluded from the formulation presented above and Figure 3.3 that this case exhibits the largest harmonic amplitude $A_{m}=A_{\max }$ at the gear mesh order $\left(H_{m}=\omega_{m} / \omega_{c}=Z_{r}\right)$. Two sideband orders of $H=\left(\omega_{m} \pm N \omega_{c}\right) / \omega_{c}=H_{m} \pm N$ and equal amplitude $\frac{1}{2} A_{m}$ are the only significant sidebands in this case.

\section{Case (ii): Equally-spaced planets and sequentially phased gear meshes}

This case is defined as 


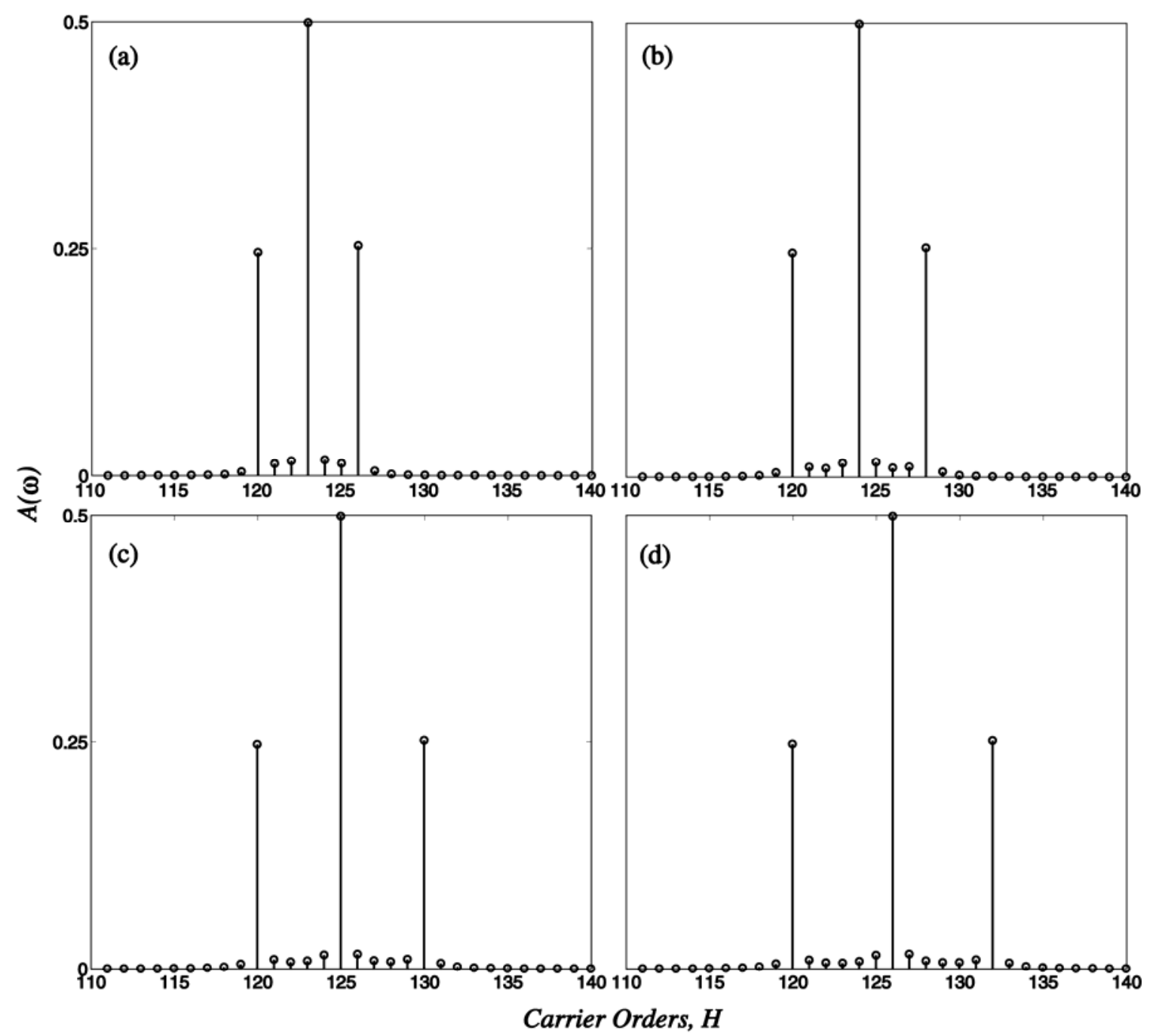

Figure 3.3: Theoretical $A(\omega)$ spectra for four different in-phase and equally-spaced planetary gear sets; (a) $N=3, Z_{r}=123$ and $Z_{s}=72$, (b) $N=4, Z_{r}=124$ and $Z_{s}=72$, (c) $N=5, Z_{r}=125$ and $Z_{s}=70$, and (d) $N=6, Z_{r}=126$ and $Z_{s}=72$. 


$$
\psi_{i}=\frac{2 \pi(i-1)}{N}, \quad \frac{Z_{r} \psi_{i}}{2 \pi} \neq n, \quad \text { and } \quad \sum_{i=1}^{N} Z_{r} \psi_{i}=m \pi \quad(m, n=\text { integers }) .
$$

Here, the second condition indicates that the planets are not in phase $\left(Z_{r} / N \neq\right.$ integer for equally spaced planets). Meanwhile, the third condition states that $F_{i}(t)$ in Eq. (3.3) are sequentially phased, i.e., sum of the phase angles is an integer multiple of $\pi$. This is indeed one of the most common conditions used in product applications since it was shown to result in lower vibration and noise levels [3.21, 3.22]. It can also be stated that this is the only other possible phasing condition for equally-spaced systems other than inphase condition.

Figures 3.4(a-d) illustrate $A(\omega)$ spectra of four different planetary gear sets (again with $C F_{1}=1$ ), respectively, each satisfying conditions defined by Eq. (3.12): (a) $N=3$, $Z_{r}=125$ and $Z_{S}=73$, (b) $N=4, Z_{r}=126$ and $Z_{s}=74$, (c) $N=5, Z_{r}=126$ and $Z_{s}=74$, and (d) $N=6, Z_{r}=122$ and $Z_{s}=70$. For instance, for gear set (a), $Z_{r} / 3=125 / 3=41 . \overline{6}, Z_{r} \psi_{1}=0, Z_{r} \psi_{2}=(125) \frac{2 \pi}{3} \equiv \frac{4 \pi}{3}$ and $Z_{r} \psi_{3}=(125) \frac{4 \pi}{3} \equiv \frac{2 \pi}{3}$ such that the third condition in Eq. (3.12) is also satisfied, i.e. $\sum_{i=1}^{3} Z_{r} \psi_{i}=0+\frac{4 \pi}{3}+\frac{2 \pi}{3}=2 \pi$

In this case, it is not possible mathematically to reduce $a(t)$ to a sum of harmonic terms as it was done for Case (i) since each $T_{C} / N$ time segment associated with each planet has a different phase angle. Yet, the behavior presented by Figure 3.4 has several well-defined traits: 


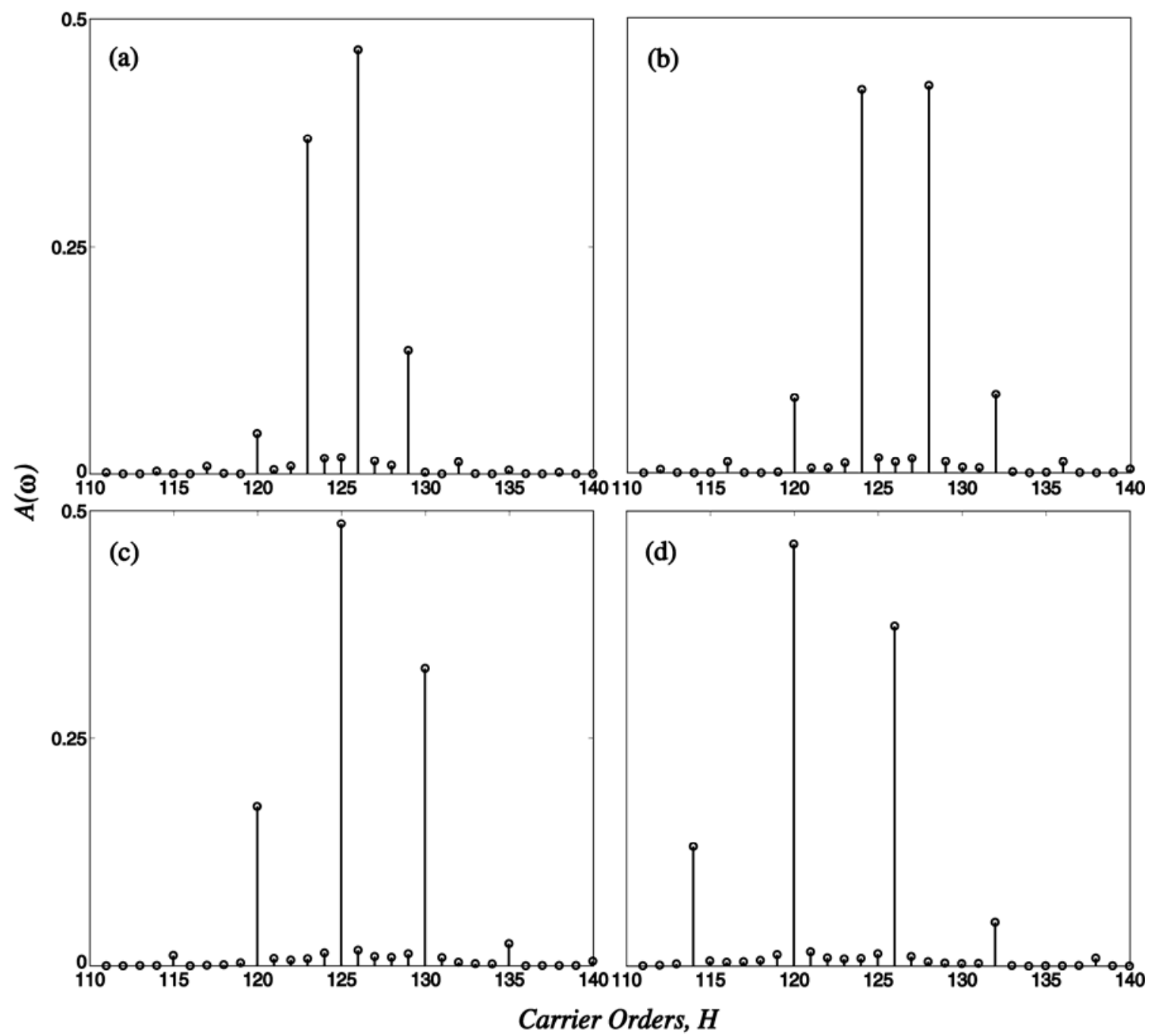

Figure 3.4: Theoretical $A(\omega)$ spectra for four different sequentially-phased and equallyspaced planetary gear sets; (a) $N=3, Z_{r}=125$ and $Z_{s}=73$, (b) $N=4, Z_{r}=126$ and $Z_{s}=74$, (c) $N=5, Z_{r}=126$ and $Z_{s}=74$, and (d) $N=6, Z_{r}=122$ and $Z_{s}=70$. 
(i) In direct contrast to Case (i), gear sets in this group exhibit nearly zero amplitude $\left(A_{\mathrm{m}} \approx 0\right)$ at the mesh harmonic order $H_{m}$. This is evident from all four cases considered in Figure 3.4.

(ii) All significant harmonic orders with sizable amplitudes are at orders $H=n N$ ( $n$ : integer) in the vicinity of mesh harmonic order $H_{m}$, i.e, they all appear at integer multiples of the number of planets $N$. In Figure 3.4(a), for instance, for $N=3$, $Z_{r}=125$ and $Z_{s}=73$, there are 4 harmonics with large amplitudes at orders of $120,123,126$ and 129, corresponding to $H=40 N, 41 N, 42 N$ and $43 N$. The same is true for the other three cases as well.

(iii) Sidebands are mostly not symmetric about $H_{m}$. The harmonic order with the largest amplitude is the order $H=n N$ that is closest to $H_{m}$, i.e. $A \approx A_{\max }$ for the order satisfying the condition $\left|H_{\max }-H_{m}\right|<\frac{1}{2} N$. In Figure 3.4(a), order $H_{\max }=42 \mathrm{~N}=126$ satisfy this condition, while it is $H_{\max }=25 \mathrm{~N}=125$ for the third example in Figure 3.4(c) where $H_{m}=126$.

(iv) A special condition rises when the remainder of the ratio $Z_{r} / N$ is equal to $\frac{1}{2}$. Even numbers for $N$ and $Z_{r}$ are necessary (but not sufficient) conditions for this situation. As an example of this, Figure 3.4(b) exhibits harmonic orders that are symmetric about $H_{m}$ and $A \approx A_{\max }$ at two orders where $\left|H_{\max }-H_{m}\right|=\frac{1}{2} N$. 
Planetary gear sets satisfying Case (ii) conditions seems to be the only ones considered in the previous planetary sideband studies including those by McFadden and Smith [3.6] and McNames [3.7]. These earlier studies reported that the dominant harmonic orders in the vicinity of the mesh are distributed apart by $N$ orders at integer multiples of $N$ in agreement with the first observation above.

\section{Case (iii): Unequally-spaced planets and in-phase gear meshes}

This condition satisfies the following:

$$
\psi_{i} \neq \frac{2 \pi(i-1)}{N} \quad \text { and } \quad \frac{Z_{r} \psi_{i}}{2 \pi}=n \quad(n=\text { integers })
$$

Here $F_{i}(t)$ are in phase in spite of the fact that planet spacing is not equal (but still meets the assembly requirements, i.e. all $\psi_{i}$ are integer multiples of $\lambda$ ). Four examples meeting these conditions are analyzed in Figure 3.5: (a) $N=3, Z_{r}=126$ and $Z_{s}=72$ with $\psi_{i}=0, \frac{2 \pi}{3}$ and $1 . \overline{2} \pi\left(\psi_{i}=0,120^{\circ}\right.$ and $\left.220^{\circ}\right)$ where planet- 3 moved $20^{\circ}$ from its equal spacing position, (b) $N=4, \quad Z_{r}=120$ and $Z_{S}=72 \quad$ with $\psi_{i}=0,0.4167 \pi, \pi$ and $1.4167 \pi\left(\psi_{i}=0,75^{\circ}, 180^{\circ}\right.$ and $\left.255^{\circ}\right)$ corresponding to an inphase "X-shaped" arrangement where there are two diametrically-opposed planet pairs, (c) $N=5, \quad Z_{r}=145$ and $Z_{S}=75$ with $\psi_{i}=0,0.4276 \pi, 0.8276 \pi, 1.2 \pi$ and $1.6 \pi$ $\left(0,76.97^{\circ}, 148.97^{\circ}, 216^{\circ}\right.$ and $\left.288^{\circ}\right)$ where planets 2 and 3 are positioned away from 


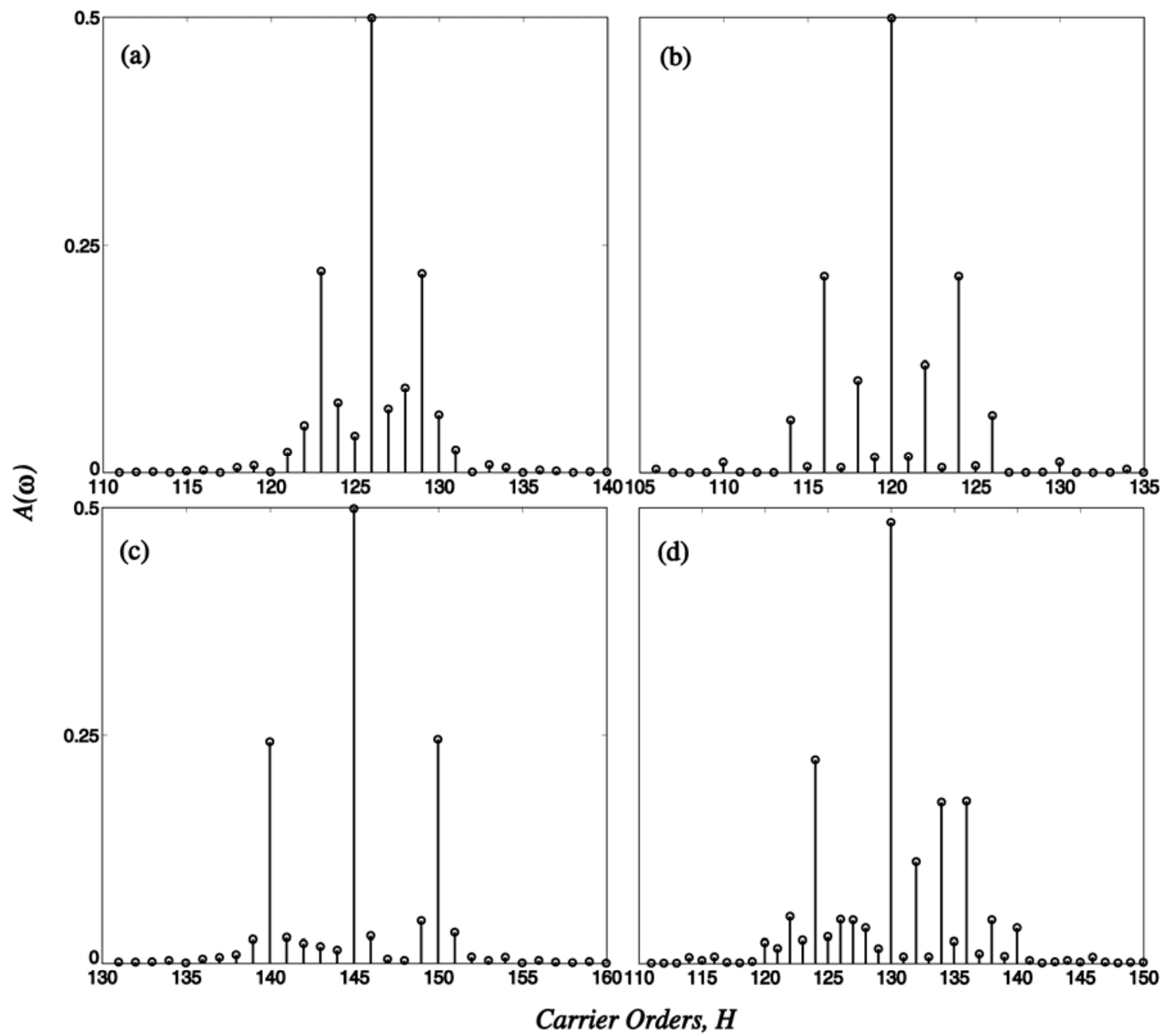

Figure 3.5: Theoretical $A(\omega)$ spectra for four different in-phase and unequally-spaced planetary gear sets; (a) $N=3, Z_{r}=126$ and $Z_{s}=72$ with $\psi_{i}=0, \frac{2 \pi}{3}$ and $1 . \overline{2} \pi$, (b) $N=4, Z_{r}=120$ and $Z_{s}=72$ with $\psi_{i}=0,0.4167 \pi, \pi$ and $1.4167 \pi$, (c) $N=5$, $Z_{r}=145$ and $Z_{s}=75$ with $\psi_{i}=0,0.4276 \pi, 0.8276 \pi, 1.2 \pi, 1.6 \pi$, and (d) $N=6$, $Z_{r}=130, Z_{s}=70, \psi_{i}=0,0.4 \pi, 0.74 \pi, \pi, 1.37 \pi$ and $1.74 \pi$. 
their equal spacing angles, and (4) $N=6, \quad Z_{r}=130, \quad Z_{s}=70$, $\psi_{i}=0,0.4 \pi, 0.74 \pi, \pi, 1.37 \pi$ and $1.74 \pi \quad\left(0,72^{\circ}, 133.2^{\circ}, 180^{\circ}, 246.6^{\circ}\right.$ and $\left.313.2^{\circ}\right)$ All of these cases satisfy conditions given by Eq. (3.13).

The frequency spectra of gear sets having this design condition have the following common features:

(i) The harmonic order with the largest amplitude is the gear mesh harmonic $H_{m}$ with $A_{\max }=A_{m}$ as in Case (i). In all four spectra shown in Figure 3.5, order $H=H_{m}=Z_{r}$ has $A_{\max }=A_{m} \approx \frac{1}{2}$.

(ii) The harmonic orders at $H_{m} \pm N$ are always the most significant sidebands that are nearly symmetric about $H_{m}$. For instance, orders 140 and 150 in Figure 3.5(c) are such sidebands that are symmetric about the mesh order $H_{m}=145$.

(iii) If both $N$ and $Z_{r}$ are even numbers, then several even orders at $H_{m} \pm 2 n$ dominate the spectrum as shown in Figure 3.5(b) and (d). On the other hand, if $N$ is odd, then sidebands appear at many of orders $H_{m} \pm n$, as in Figures 3.5(a) and (c).

(iv) As a special condition for this case, if the planets are positioned in diametricallyopposed pairs ( $N=$ even and the spacing between each planet in the pair is $180^{\circ}$ ), then the sideband orders exist at even multiples of the carrier order at orders $H_{m} \pm 2 n$ (n: integer), as it the case in Figure 3.5(b). 


\section{Case (iv): Unequally-spaced planets and sequentially phased gear meshes}

This case is defined mathematically as:

$$
\psi_{i} \neq \frac{2 \pi(i-1)}{N}, \quad \frac{Z_{r} \psi_{i}}{2 \pi} \neq n \quad \text { and } \quad \sum_{i=1}^{N} Z_{r} \psi_{i}=m \pi \quad(m, n=\text { integers })
$$

$A(\omega)$ spectra for four different examples of this case are shown in Figure 3.6.

Figure 3.6(a) is for a system having $N=3, Z_{r}=125, Z_{s}=73, \psi_{i}=0,0 . \overline{5} \pi$ and $1 . \overline{4} \pi$ $\left(\psi_{i}=0,100^{\circ}\right.$ and $\left.260^{\circ}\right)$. Second example is a 5 planet gear set with $Z_{r}=125, Z_{s}=73$, and $\psi_{i}=0,0.404 \pi, 0.798 \pi, 1.202 \pi$ and $1.596 \pi \quad\left(\psi_{i}=0,72.727^{\circ}, 143.636^{\circ}\right.$,

$216.364^{\circ}$ and $287.273^{\circ}$ ) and has the spectra shown in Figure 3.6(b). Third example is a 4-planet gear set with $Z_{r}=126, Z_{s}=74$, and $\psi_{i}=0,0.54 \pi, 0.97 \pi$, and $1.57 \pi$ $\left(\psi_{i}=0,97.2^{\circ}, 174.6^{\circ}, 282.6^{\circ}\right)$ whose predicted spectrum is shown in Figure 3.6(c). The last example represented in Figure 3.6(d) is a 6-planet gear set with $Z_{r}=176$, $Z_{S}=74$, and the planet position angles are $\psi_{i}=0,0.376 \pi, 0.704 \pi, \pi, 1.376 \pi$ and $1.68 \pi$ $\left(\psi_{i}=0,67.68^{\circ}, 126.72^{\circ}, 180^{\circ}, 247.68^{\circ}, 302.4^{\circ}\right)$.

The spectra corresponding to such gear sets are more complex, with the following common features:

(i) In general, sideband harmonics appear at several integer multiples of the carrier order at $H_{m} \pm n\left(n\right.$ : integer) in an asymmetric way around $H_{m}$. The number of 


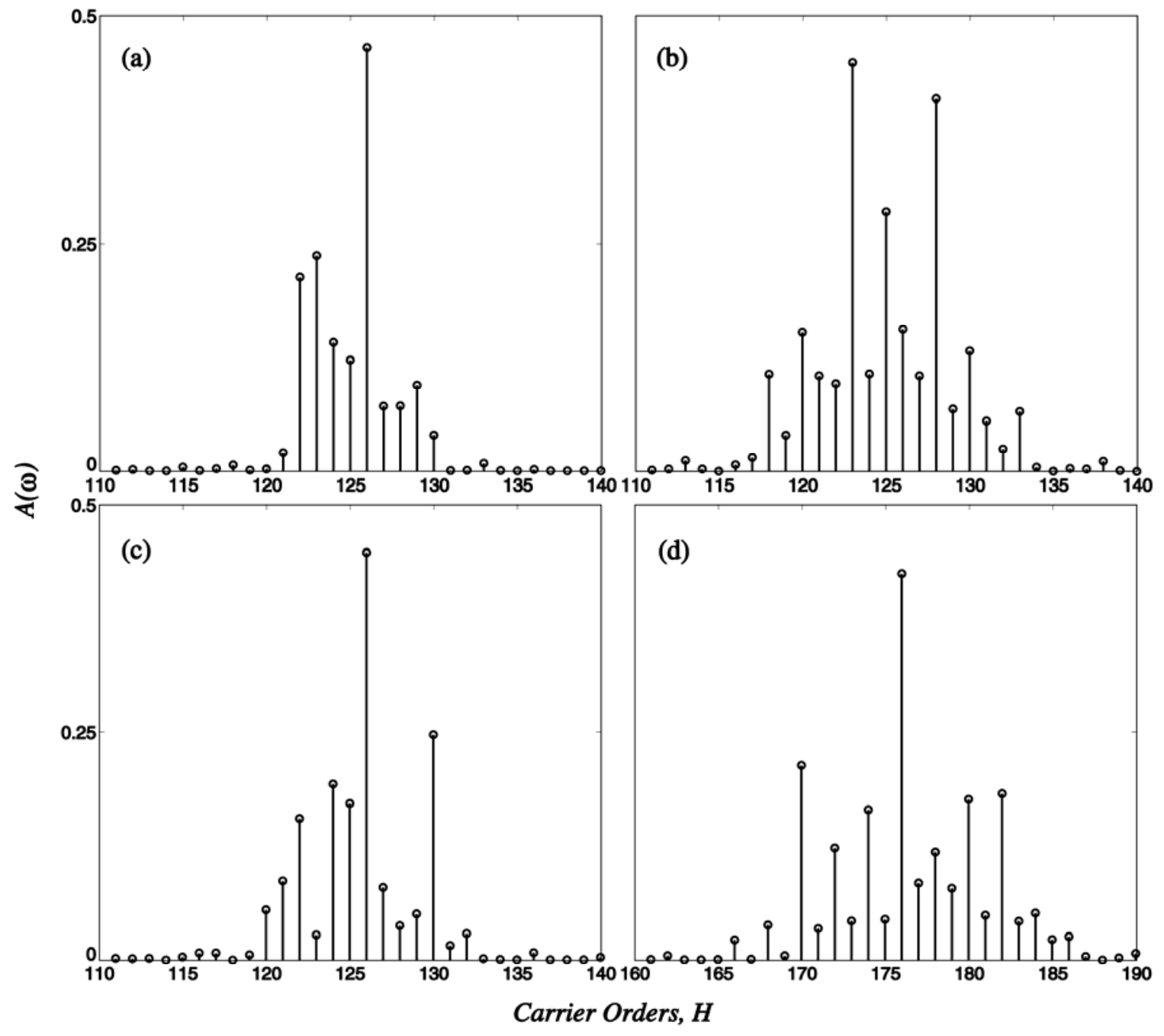

Figure 3.6: Theoretical $A(\omega)$ spectra for four different sequentially-phased and unequally-spaced planetary gear sets; (a) $N=3, Z_{r}=125, Z_{s}=73, \psi_{i}=0,0 . \overline{5} \pi, 1 . \overline{4} \pi$, (b) $N=5, Z_{r}=125, Z_{s}=73, \psi_{i}=0,0.404 \pi, 0.798 \pi, 1.202 \pi$ and $1.596 \pi$, (c) $N=4$, $Z_{r}=126, Z_{s}=74$, and $\psi_{i}=0,0.54 \pi, 0.97 \pi$, and $1.57 \pi$, and (d) $N=6, Z_{r}=176$, $Z_{S}=74$, and $\psi_{i}=0,0.376 \pi, 0.704 \pi, \pi, 1.376 \pi$ and $1.68 \pi$. 
sidebands with non-zero amplitudes is quite high in each case considered in Figure 3.6. For instance, there are about 15 sidebands with sizable amplitude in the spectrum of the third gear set.

(ii) In regards to the mesh harmonic order, $A_{\max }=A_{m}$ when both $Z_{r}$ and $N$ are even numbers, as exemplified by Figures 3.6(c) and (d) where $H_{\max }=H_{m}=Z_{r}$. For such systems, if there is at least one diametrically-opposed planet pair, then the sidebands at even numbered orders are dominant.

(iii) If both $Z_{r}$ and $N$ are odd numbers, then the gear mesh harmonic order, while non-zero, does not represent the largest amplitude.

\section{Case (v): Unequally-spaced planets and arbitrarily phased gear meshes}

The last and perhaps the most arbitrary case is obtained when the planets are positioned unequally at multiples of $\lambda$ and planet phasing is arbitrary (neither in-phase nor sequentially-phased) such that

$$
\psi_{i} \neq \frac{2 \pi(i-1)}{N}, \quad \frac{Z_{r} \psi_{i}}{2 \pi} \neq n \quad \text { and } \quad \sum_{i=1}^{N} Z_{r} \psi_{i} \neq m \pi \quad(m, n=\text { integers }) .
$$

Similar to the previous cases, four examples are chosen here to illustrate this case in Figure 3.7. The first example is a 3-planet system with $Z_{r}=125, Z_{s}=73$ and $\psi_{i}=0,0 . \overline{55} \pi$ and $1.384 \pi \quad\left(\psi_{i}=0,100^{\circ}\right.$ and $\left.249.13^{\circ}\right)$ and exhibiting a spectrum as 
shown in Figure 3.7(a). The second example is a "X-shaped" 4-planet gear set having $Z_{r}=125, \quad Z_{s}=73$ and planet position angles $\psi_{i}=0,0.505 \pi, \pi$ and $1.505 \pi$ $\left(\psi_{i}=0,90.909^{\circ}, 180^{\circ}\right.$ and $\left.270.909^{\circ}\right)$. In this case, phase angles $Z_{r} \psi_{i}=0,1.1325 \pi, \pi$ and $0.1312 \pi$ and the sum of the phase angles is obviously not an integer multiple of $\pi$. The corresponding spectrum for this gear set is given in Figure 3.7(b). The other example corresponding to the spectrum shown in Figure 3.7(c) is a gear set having $N=5, Z_{r}=127, Z_{s}=73$ and $\psi_{i}=0,0.42 \pi, 0.83 \pi, 1.24 \pi$ and $1.65 \pi$ $\left(\psi_{i}=0,75.6^{\circ}, 149.4^{\circ}, 223.2^{\circ}\right.$ and $\left.297^{\circ}\right)$. As a last example for this case, a 6-planet gear set with $Z_{r}=125, \quad Z_{s}=73$, and $\psi_{i}=0,0 . \overline{34} \pi, 0 . \overline{68} \pi, 1 . \overline{03} \pi, 1 . \overline{34} \pi$, and $1 . \overline{66} \pi$ $\left(0,61.8181^{\circ}, 123.6362^{\circ}, 185.4543^{\circ}, 241.8181^{\circ}\right.$ and $\left.300^{\circ}\right)$ is chosen. This irregularlyspaced gear set exhibits a frequency spectrum as shown in Figure 3.7(d).

The spectra shown in Figure 3.7 illustrate a very rich sideband activity for gear sets satisfying the conditions for this case, with sideband orders at $H_{m} \pm n$ that are asymmetric about $H_{m}$. It is not possible to draw many conclusions from Figure 3.7, except to say that even numbered sideband orders are stronger for an even $N$. Meanwhile, if $N$ is an odd number, then sidebands appear at almost every order neighboring $H_{m}$. 


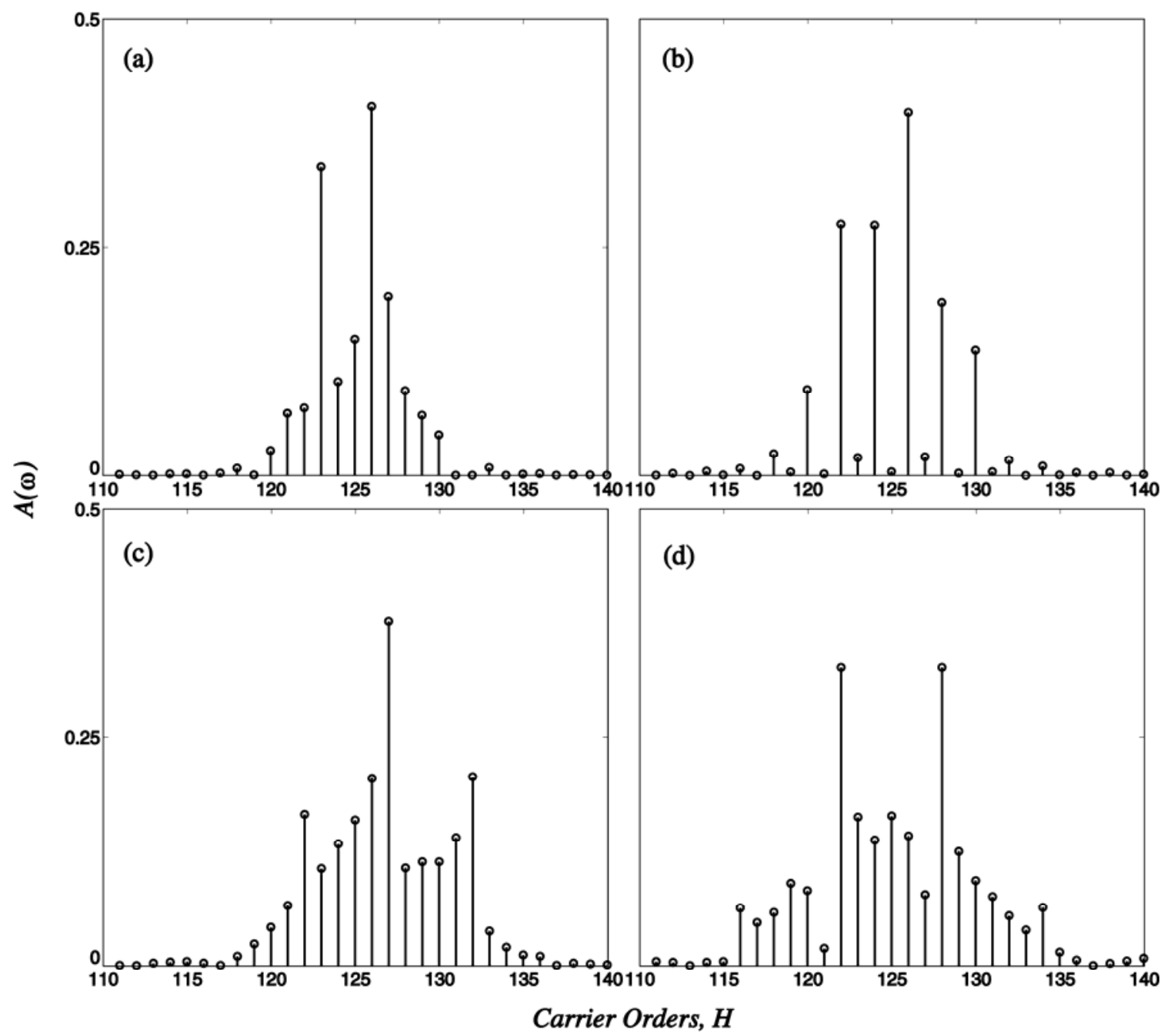

Figure 3.7: Theoretical $A(\omega)$ spectra for four different arbitrarily-phased and unequallyspaced planetary gear sets; (a) $N=3, Z_{r}=125, Z_{s}=73$ and $\psi_{i}=0,0 . \overline{55} \pi$ and $1.384 \pi$, (b) $N=4, Z_{r}=125, Z_{s}=73$ and $\psi_{i}=0,0.505 \pi, \pi$ and $1.505 \pi$, (c) $N=5, Z_{r}=127$, $Z_{s}=73$ and $\psi_{i}=0,0.42 \pi, 0.83 \pi, 1.24 \pi$ and $1.65 \pi$, and (d) $N=6, Z_{r}=125, Z_{s}=73$, and $\psi_{i}=0,0 . \overline{34} \pi, 0 . \overline{68} \pi, 1 . \overline{03} \pi, 1 . \overline{34} \pi, 1 . \overline{66} \pi$. 


\subsection{Experimental Verification of the Modulation Model}

\subsubsection{Categorization of the Experimental Planetary Gear Sets based on Sideband Patterns}

The five distinct cases identified in Section 3.3 would require design and procurement of five different planetary gear sets with appropriate values for the $N+2$ parameters $\left(N, Z_{r}, \psi_{1}, \psi_{2}, \ldots, \psi_{N}\right)$ so that each gear set satisfies the conditions defined by one of the Eq. (3.10), (3.12)-(3.15). Since this is very time consuming and costly, the experimental study described in Chapter 2 considered the same baseline gear set design with $Z_{s}=73$ and $Z_{r}=125$. The remaining parameters $\left(N, \psi_{1}, \psi_{2}, \ldots, \psi_{N}\right)$ were varied to obtain three different gear sets, each representing a different case defined in Section 3.3. These three cases are specifically designed and they essentially represent the most complex and frequently used cases among the others as described below.

$\underline{\text { Gear Set } A}$. With $Z_{S}=73$ and $Z_{r}=125$, this three-planet gear set $(N=3)$ has planets at $\psi_{i}=0, \frac{2 \pi}{3}, \frac{4 \pi}{3}$ since equally spaced planet condition is met, i.e. $\left(Z_{r}+Z_{S}\right) / N=(73+125) / 3=66=$ integer (in other words, all $\psi_{i}$ are integer multiples of the least mesh angle $\left.\lambda=360 /\left(Z_{S}+Z_{r}\right)=1.8182^{\circ}\right)$. In addition, planet gear meshes are sequentially phased since $Z_{r} / 3=125 / 3=41 . \overline{6}, Z_{r} \psi_{1}=0, Z_{r} \psi_{2}=(125) \frac{2 \pi}{3} \equiv \frac{4 \pi}{3}$ and $Z_{r} \psi_{3}=(125) \frac{4 \pi}{3} \equiv \frac{2 \pi}{3}$. Therefore, Eq. (3.12) is satisfied and this gear set represents Case 
(ii) in the previous section in terms of its sideband activity. This gear set is indeed the example gear set (a) for this case whose predicted spectrum is shown in Figure 3.4(a).

Gear Set B. Again, with $Z_{S}=73$ and $Z_{r}=125$ (the same gears), this 5-planet gear set $(N=5)$ was obtained by designing a 5-planet carrier with $\psi_{i}=0,0.404 \pi, 0.798 \pi, 1.202 \pi, 1.596 \pi\left(\psi_{i}=0,72.727^{\circ}, 143.636^{\circ}, 216.364^{\circ}, 287.273^{\circ}\right)$.

Here, the equal spacing condition is not satisfied since $(73+125) / 5=39.6$ (not an integer) while assembly condition is met since all $\psi_{i}$ are integer multiples of the least mesh angle of $\lambda$. In addition $\sum_{i=1}^{5} Z_{r} \psi_{i} \equiv 0+0.505 \pi+1.747 \pi+0.253 \pi+1.495 \pi=4 \pi$, indicating that the gear meshes are sequentially phased. Accordingly, this gear set satisfies Eq. (3.14) and represents an unequally-spaced and sequentially phased gear set of Case (iv) type. Figure 3.6(b) illustrates the predicted acceleration spectra for this planetary gear set.

Gear Set C. The last experimental planetary gear set was obtained with the same gears $\left(Z_{s}=73\right.$ and $\left.Z_{r}=125\right)$, and an X-shaped 4-planet carrier $(N=4)$. In this arrangement, the planets are at angles of $\psi_{i}=0,0.505 \pi, \pi$ and $1.505 \pi$ $\left(\psi_{i}=0,90.909^{\circ}, 180^{\circ}\right.$ and $\left.270.909^{\circ}\right)$. Here, the planet pairs $1-3$ and $2-4$ are diametrically opposed while the equal spacing and special (in-phase or sequentially phased) phasing conditions are not met as $Z_{r} \psi_{i}=0,1.131 \pi, \pi, 0.131 \pi$. Accordingly, this case represents an unequally spaced and arbitrarily phased gear set of Case (v) type, 
satisfying the mathematical conditions of Eq. (3.15). The predicted sideband activity for this gear set was given in Figure 3.7(b).

\subsubsection{Experimental Results}

Figure 3.8 shows the measured order spectra from Gear Set A (Case (ii) type with equally spaced and sequentially phased planets) at various $T_{S}$ and $\Omega_{S}$ values, focusing on the activity around $H_{m}$. The first major observation from these spectra is that no harmonic order is apparent at $H_{m}=125$, i.e. $A_{m} \approx 0$. All significant harmonic orders with sizable amplitudes are at orders $H=n N=\ldots, 117,120,123,126,129,132, \ldots$ in the vicinity of mesh harmonic order $H_{m}$. Sidebands are not symmetric about $H_{m}$. All these observations agree with the predicted spectra of Figure 3.4(a) for this gear set and the sideband behavior established in Section 3.3 for Case (ii) gear sets. One difference is that predicted harmonic order with the largest amplitude was the order $H=n N=126$ that is closest to $H_{m}=125$ while $A_{\max }$ is observed at $H=123$ in the measured spectra shown in Figure 3.8. It is also noted that relative amplitudes of the sideband orders vary with $T_{S}$ and $\Omega_{s}$ due to dynamic effects that were not included in this model.

Similarly, Figure 3.9 shows the spectra from Gear Set B at four different $T_{S}$ and $\Omega_{s}$ combinations. For this Case (iv) type gear set (unequally spaced and sequentially phased), the measured sideband activity carries the most of the common features predicted from the analytical model (Fig. 3.6(b)). 


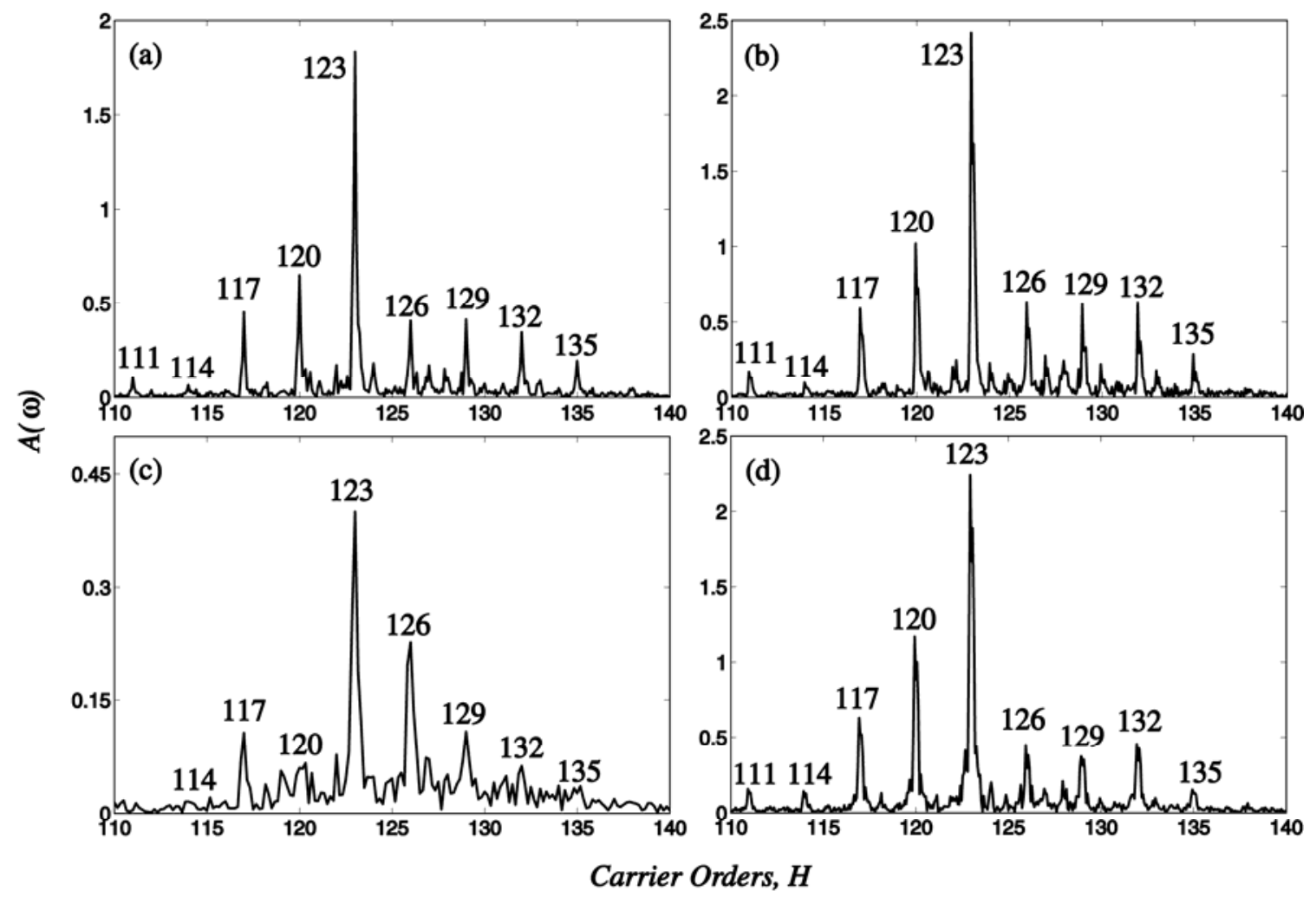

Figure 3.8: Examples of measured $A(\omega)$ order spectra for the Gear Set $\mathrm{A}$ at different torque and speed conditions; (a) $T_{S}=500 \mathrm{Nm}, \Omega_{S}=400 \mathrm{rpm}$, (b) $T_{S}=600 \mathrm{Nm}$, $\Omega_{s}=500 \mathrm{rpm}$, (c) $T_{S}=200 \mathrm{Nm}, \Omega_{s}=200 \mathrm{rpm}$, and (d) $T_{S}=1000 \mathrm{Nm}, \Omega_{s}=500 \mathrm{rpm}$. 


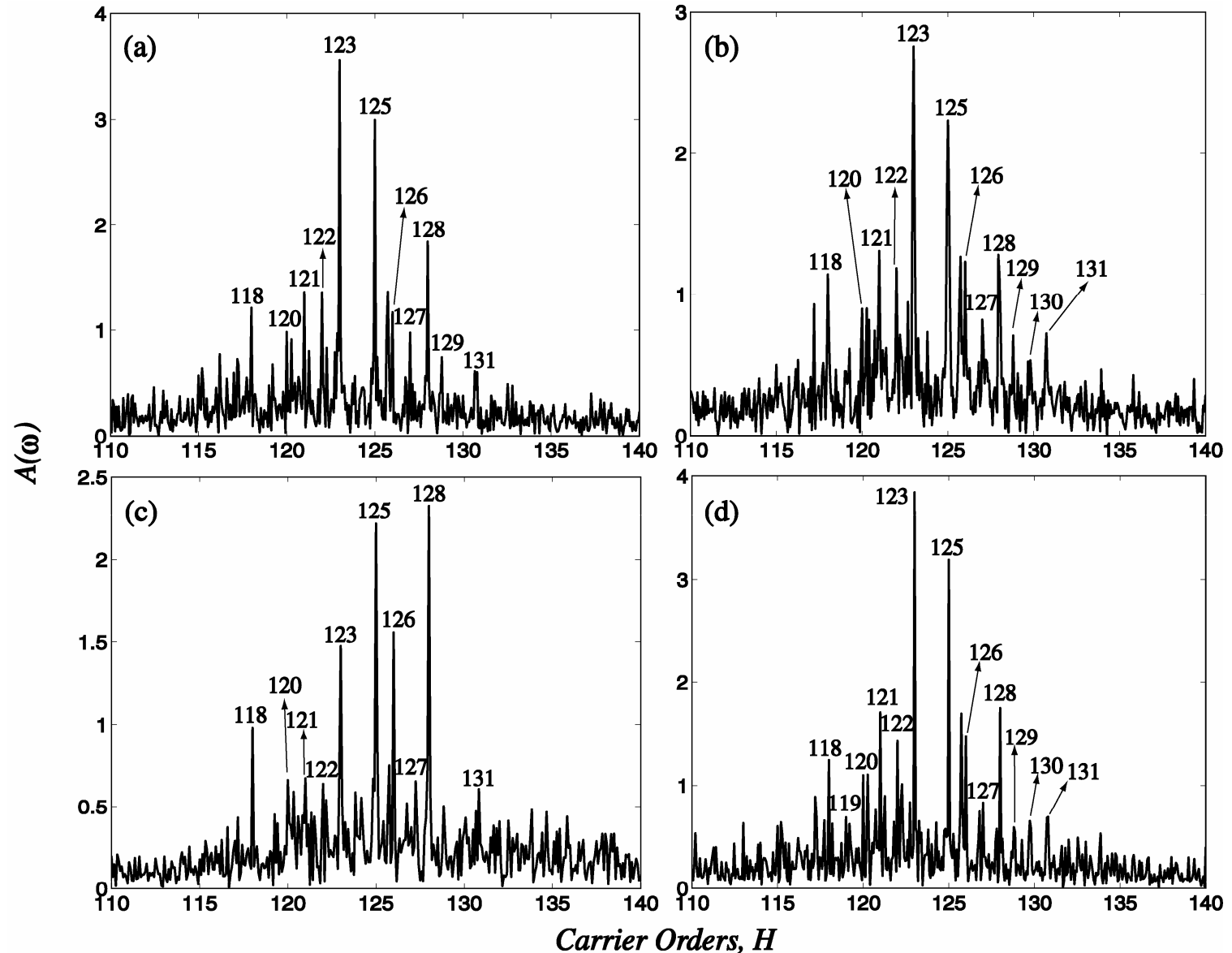

Figure 3.9: Examples of measured $A(\omega)$ order spectra for the Gear Set B at different toque and speed conditions; (a) $T_{S}=400 \mathrm{Nm}, \Omega_{s}=500 \mathrm{rpm}$, (b) $T_{S}=500 \mathrm{Nm}, \Omega_{s}=500$ $\mathrm{rpm}$, (c) $T_{S}=800 \mathrm{Nm}, \Omega_{s}=400 \mathrm{rpm}$, and (d) $T_{S}=1000 \mathrm{Nm}, \Omega_{S}=500 \mathrm{rpm}$. 

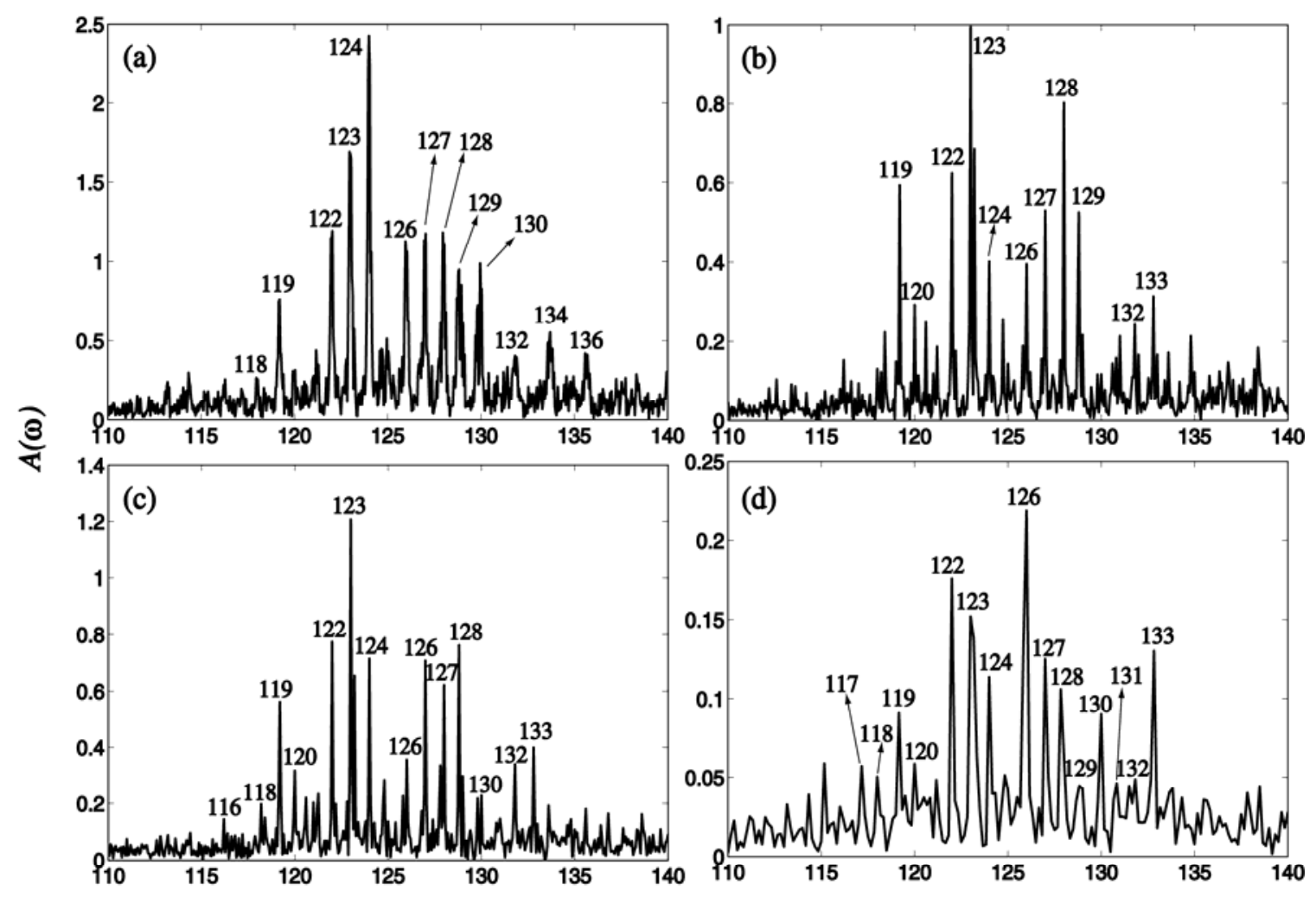

Carrier Orders, $\boldsymbol{H}$

Figure 3.10: Examples of measured $A(\omega)$ order spectra for the Gear Set $\mathrm{C}$ at different torque and speed conditions; (a) $T_{S}=200 \mathrm{Nm}, \Omega_{\mathrm{S}}=800 \mathrm{rpm}$, (b) $T_{S}=400 \mathrm{Nm}$, $\Omega_{S}=500 \mathrm{rpm}$, (c) $T_{S}=600 \mathrm{Nm}, \Omega_{s}=500 \mathrm{rpm}$, and (d) $T_{S}=1000 \mathrm{Nm}, \Omega_{S}=200 \mathrm{rpm}$. 
Specifically, sideband harmonics appear at several integer multiples of the carrier order at $H_{m} \pm n$ ( $n$ : integer) in an asymmetric way around $H_{m}$. The number of sidebands with tangible amplitudes is rather high (10 to 15$)$. In addition, as both $Z_{r}$ and $N$ are odd numbers, the gear mesh harmonic order $H_{m}=125$, while non-zero, does not represent the largest amplitude.

Finally in Figure 3.10, four measured $A(\omega)$ spectra are shown for Gear Set C (Case (v) type with unequally spaced and arbitrarily phased planets). In line with the prediction of Figure 3.7(b), these measured spectra exhibit a rich sideband activity with sideband orders at $H_{m} \pm n$ that are asymmetric about $H_{m}$. The gear mesh harmonic order has small amplitudes as in predictions of Figure 3.7(b). Only main difference between these measurements and the predictions from the simplified model is that experiments provide a full sideband spectrum with components at $H_{m} \pm n$ while the predicted spectrum for this $\mathrm{X}$-shaped 4-planet gear set ( $N$ : even) reveals more dominant even sideband orders. This suggests that there might be other mechanisms that are not included in the simplified analysis such as frequency modulations and unequal planet load sharing characteristics.

\subsection{Conclusion}

In this chapter, modulation sidebands of planetary gear sets having a rotating carrier were investigated analytically and experimentally. First, a simplified analytical model was proposed to predict the modulation sideband orders as well as relative 
amplitude distributions in the vicinity of the mesh frequency harmonic orders. The model considered the number of planets, planet position angles and the number of teeth of the stationary gear (sun or ring) as the sole parameters defining the resultant sideband activity. The model was used to simulate a wide range of gear sets to show that any planetary gear set can be classified in one of five distinct groups: (i) equally-spaced and in-phase planets, (ii) equally-spaced and sequentially phased planets, (iii) unequallyspaced and in-phase planets, (iv) unequally-spaced and sequentially phased planets, and (v) unequally-spaced and arbitrarily phased planets. General sideband behavior unique to each of these cases were characterized and demonstrated through simulation of various gear sets. Based on this behavior, other general conclusions can be made as follows:

(i) In a sequentially-phased planetary gear set, sidebands are always asymmetrically distributed about the mesh orders.

(ii) A dominant (and maximum) mesh harmonic amplitude is achieved only when the planet meshes are in phase $\left(Z_{r} / N=\right.$ integer $)$.

(iii) Symmetric sidebands in both frequencies and amplitudes about the mesh frequency are possible only when the gear set is in-phase and equally-spaced.

The experimental study described earlier included gear sets from three of the five distinct cases identified by the model. Comparison of the measured spectra to the predictions and general behavior presented in Section 3.2 indicated that the model, while simple in many aspects, is capable of capturing the bulk of the sideband behavior. Measurements also revealed qualitatively different behavior for the three gear sets 64 
considered in support of the classification of planetary gear set arrived at based on this model. With this, the general trends unique to each of the five cases of gear sets can be deemed valid, as they were observed in the experimental data as well. They also point to other influences caused by the dynamic effects at different speeds as well as the changes in gear mesh excitations with the torque transmitted. Moreover, there are additional sidebands that can not be captured by the model described here, which are anticipated to be caused by manufacturing errors within the planetary members. Accordingly, Chapter 4 focuses incorporating this analytical model with a discrete parameter, nonlinear timevarying dynamic model to predict the gear mesh dynamic forces and sidebands associated with manufacturing errors. Experimental data from the sideband database of Chapter 2 will be used to verify the model described in Chapter 4 as well. 


\section{References for Chapter 3:}

[3.1] G. W. Blankenship, R. Singh, Analytical solution for modulation sidebands associated with a class of mechanical oscillators, Journal of Sound and Vibration, 179 (1) (1995) 13-36.

[3.2] L. Bellomonte, I. Guastella, and R. M. Sperandeo-Mineo, Mechanical models of amplitude and frequency modulation, European Journal of Physics, 26 (2005) 409-422.

[3.3] H. Ligata, A. Kahraman, and A. Singh, An experimental study on the influence of manufacturing errors on the planetary gear stresses and planet load sharing, ASME Journal of Mechanical Design, 130 (4) (2008) 041701-1 - 041701-9.

[3.4] A. Bodas, A. Kahraman, Influence of carrier and gear manufacturing errors on the static load sharing behavior of planetary gear sets, JSME International Journal, Series C, 47-3 (2004) 908-915.

[3.5] T. Hidaka, Y. Terauchi, and K. Ishioka, Dynamic behavior of planetary gear (2nd report: displacement of sun gear and ring gear), Bulletin of the JSME, 19-138 (1976) 1563-1570.

[3.6] P. D. McFadden and J. D. Smith, An explanation for the asymmetry of the modulation sidebands about the tooth meshing frequency in epicyclic gear vibration, Proceedings of the Institution of Mechanical Engineers, 199 C1 (1985) $65-70$. 
[3.7] J. McNames, Fourier series analysis of epicyclic gearbox vibration, ASME Journal of Vibration and Acoustics, 124 (2002) 150-152.

[3.8] C. E. Fair, Synchronous sampling sideband orders from helical planetary gear sets, MS Thesis, Virginia Polytechnic Institute and State University, 1998.

[3.9] A. L. Gu, R. H. Badgley, and T. Chiang, Planet-pass-induced vibration in planetary reduction gears, ASME Design Engineering Technical Conference, New York NY USA, October 1974, Paper No: 74-DET-93.

[3.10] A. L. Gu, R. H. Badgley, Prediction of vibration sidebands in gear meshes, ASME Design Engineering Technical Conference, New York NY USA, October 1974, Paper No: 74-DET-95.

[3.11] P. P. Schon, Unconditionally convergent time domain adaptive and timefrequency techniques for epicyclic gearbox vibration, ME Thesis, The University of Pretoria, June 2005.

[3.12] A. M. Mitchell, F. B. Oswald, and H. H. Coe, Testing of UH-60A helicopter transmission in NASA Lewis $2240 \mathrm{~kW}$ (3000 hp) Facility, NASA Technical Paper 2626, August 1986.

[3.13] M. Mosher, Results from a new separation algorithm for planetary gear system vibration measurements, Proceedings of ASME International Design Engineering Technical Conferences and Computers and Information in Engineering Conference, California USA, September 2005, Paper No: DETC-84404. 
[3.14] M. Mosher, Understanding vibration spectra of planetary gear systems for fault detection, Proceedings of ASME Design Engineering Technical Conferences, Illinois USA, September 2003, Paper No: PTG-48082.

[3.15] D. G. Lewicki, J. J. Coy, Vibration characteristics of OH-58A helicopter main rotor transmission, NASA Technical Paper 2705, 1987.

[3.16] A. Saxena, B. Wu, and G. Vachtsevanos, A methodology for analyzing vibration data from planetary gear systems using complex morlet wavelets, 2005 American Control Conference, Portland OR USA, June 2005, pp. 4730-4735.

[3.17] D. M. Blunt, J. A. Keller, Detection of a fatigue crack in a UH-60A planet gear carrier using vibration analysis, Mechanical Systems and Signal Processing, 20 (2006) 2095-2111.

[3.18] R. Patrick-Aldaco, A model based framework for fault diagnosis and prognosis of dynamical systems with an application to helicopter transmissions, Ph.D. Thesis, Georgia Institute of Technology, August 2007.

[3.19] J. A. Hines, D. S. Muench, J. A. Keller, and A. K. Garga, Effects of timesynchronous averaging implementations on HUMS features for UH-60A planetary carrier cracking, American Helicopter Society 61st Annual Forum, Grapevine TX USA, June 2005, Paper No: VA 22314-2538.

[3.20] J. A. Keller, P. Grabill, Vibration monitoring of UH-60A main transmission planetary carrier fault, American Helicopter Society 59th Annual Forum, Phoenix Arizona USA, May 2003. 
[3.21] A. Kahraman, Planetary gear train dynamics, ASME Journal of Mechanical Design, 116 (1994) 713-720.

[3.22] A. Kahraman, Load sharing characteristics of planetary transmissions, Mechanism and Machine Theory, 29-8 (1994) 1151-1165.

[3.23] A. Kahraman, G. W. Blankenship, Planet mesh phasing in epicyclic gear sets, Proceedings of International Gearing Conference, Newcastle UK, September 1994, pp. 99-104.

[3.24] M. Inalpolat, A. Kahraman, Dynamic modelling of planetary gears of automatic transmissions, Proceedings of the Institution of Mechanical Engineers Part K: Journal of Multi-body Dynamics, 222 (2008) 229-242.

[3.25] A. Al-shyyab, A. Kahraman, A non-linear dynamic model for planetary gear sets, Proceedings of the Institution of Mechanical Engineers Part K: Journal of Multibody Dynamics, 221 (2007) 567-576. 


\section{CHAPTER 4}

\section{MODULATION SIDEBANDS OF A PLANETARY GEAR SET WITH MANUFACTURING ERRORS}

\subsection{Introduction}

Measured vibration spectra presented in Chapter 2 were shown to contain components at frequencies around the gear mesh (tooth pass) frequency and its higher harmonics. In Chapter 3, one of the origins of some of these sideband components was proposed to be the rotation of the planet carrier that causes amplitude modulations of the dynamic forces of the gear meshes that rotate with the carrier. The analytical model proposed in Chapter 3 was shown to be reasonably effective in predicting the amplitude modulation sidebands of this kind. This model showed that the amplitude modulations (AM) of an acceleration spectrum due to the rotation of the carrier are defined by (i) the number of planets in the gear set, (ii) planet spacing angles, and (iii) number of teeth of gear components. These parameters that define relative phasing relationships among the planet meshes not only impact the force/vibration cancellation and neutralization schemes that are studied widely [4.1, 4.2], but also define the frequencies and amplitudes of certain sidebands on vibration spectra. Based on the simplified analytical 
model presented in Chapter 3, which assumes that the dynamic gear mesh forces are known up front, planetary gear sets were classified in five groups based on their sideband behavior: (i) equally-spaced and in-phase planets, (ii) equally-spaced and sequentially phased planets, (iii) unequally-spaced and in-phase planets, (iv) unequally spaced and sequentially phased planets, (v) unequally spaced and arbitrarily phased planets. General rules for sidebands for each category that are all caused by the rotation of the carrier were also established in Chapter 3, which were shown to agree with the experimental results from Chapter 2.

The measured acceleration spectra presented in Chapter 2 contained additional sideband activity that is not possible to describe solely by AM caused by the rotation of the carrier. The cause of these additional sidebands can be attributable to certain manufacturing errors of the gears. Chaari et al [4.3] developed a discrete dynamic model of a planetary gear set to predict the influence of such errors in the form of gear run-out (eccentricity) on the dynamic gear mesh forces due to changes to the gear motion transmission error excitations. Another potential cause for planetary gear set sidebands was suggested to be the unequal planet load sharing that is mostly impacted by the errors of the planet carrier. In one such study, Mark and Hines [4.4] predicted the effect of unequal planet load sharing due to a crack on the carrier planet posts on the sideband distributions.

Most of the above studies focused on AM effects. Published studies on frequency modulations (FM) did not consider planetary gear sets [4.5-4.7]. They focused on an analytical treatment of FM modulations of a class of oscillators, representative of gear 
systems. Randall [4.5], and Sweeney and Randall [4.6] qualitatively explained some of the possible sources of gear signal modulations for parallel axis gears by performing simplified simulations. They presented possible effects of manufacturing errors on the resultant vibration spectra via amplitude and phase modulations. In another study, Blankenship and Singh [4.7] studied both amplitude and angle modulations of a damped oscillator analytically and developed solution methodologies for the equations representing these types of systems. Besides these studies, there are a variety of works that deal with sidebands in the wider context of planetary gear vibrations [4.8-4.15]. The same is true for studies on planetary vibration condition monitoring for diagnostics purposes [4.15-4.17].

This chapter aims at expanding on the modeling effort presented in Chapter 3 by

(i) providing a nonlinear time-varying model of a planetary gear set to predict dynamic gear mesh forces,

(ii) formulating modulated gear mesh stiffness and gear transmission error excitations due to certain gear manufacturing errors, and

(iii) combining the dynamic model with the AM formulation proposed for carrier rotation.

For this purpose, a non-linear time-varying dynamic model will be proposed to predict modulation sidebands of planetary gear sets with manufacturing errors. The discrete dynamic model will include both periodically varying gear mesh stiffnesses and the nonlinearities associated with tooth separations. The model will formulate forms of gear 
mesh excitations that are amplitude and frequency modulated due to a class of gear manufacturing errors to predict dynamic forces at all sun-planet gear and ring-planet gear meshes. This model will further be combined with the modulation model presented in Chapter 3 that accounts for amplitude modulations due to rotation of the carrier to predict acceleration spectrum at a fixed position in the planetary transmission housing. This unified model is intended to include both AM and FM due to gear manufacturing errors as well as AM due to the rotation of the carrier to obtain a fundamental understanding of mechanisms of modulation sidebands in planetary gear sets. The model will be formulated in a general way to handle a planetary gear set with any number of planets that are spaced in any arbitrary positions. The model will be used to simulate an example planetary gear set to study the individual AM and FM contributions of various errors and arrive at rules in identifying them on a given vibration spectrum. At the end, the model predictions will be compared to a measured vibration spectrum from a planetary gearbox to demonstrate the effectiveness of the model in predicting sidebands of a planetary gear set.

The influences of unequal planet load sharing [4.18] as well as elastic deflections of gear bodies on modulation sidebands will not be included in this chapter. The flexibilities will be kept limited to the gear meshes and radial bearings. The model will focus primarily on simple planetary gear sets (each planet meshing with the same sun and ring gears). However, the same methodology can be applied to other kinematic forms of planetary gear sets such as double-planet gear sets, compound (multi-stage) planetary gear sets and complex-compound planetary gear sets [4.19] including Ravigneaux arrangements. 


\subsection{Prediction of Modulation Sidebands under Dynamic Conditions}

The modeling methodology employed here consists of two major steps. The first step is to predict the dynamic gear mesh forces by using a discrete dynamic model that includes various forms of typical manufacturing errors. As these errors modulate the excitations used for the dynamic model, the predicted dynamic mesh force spectra should be expected to contain sideband orders associated with these errors. In case of a gear set with a stationary carrier, the measured vibration spectra on the housing as well as the noise around the gearbox will have the same spectral characteristics as the dynamic mesh forces. In case of a planetary gear set with a rotating carrier, however, planets move relative to any fixed measurement location, causing additional amplitude modulations as described recently in Chapter 3. Accordingly, the second step in this methodology is to apply the amplitude modulation formulation of Chapter 3 to the dynamic gear mesh forces predicted by the dynamic model to capture the combined influence of manufacturing errors and carrier rotation on the modulation sidebands of the planetary gear set.

\subsubsection{Dynamic Model of a Planetary Gear Set}

A discrete, two-dimensional time-varying dynamic model of a planetary gear set is considered here. This model is based on an earlier model [4.20] that was developed to study dynamic planet load sharing characteristics of planetary gear systems. This model will be expanded here to include the influences of various gear and carrier related manufacturing errors. The model considers a simple planetary gear set with $N$ planets 
(typically $N=3$ to 7 for most automotive and aerospace applications) that are positioned in any angular spacing allowed by the geometric constraints. As $N$ and the spacing angles of planets $\left(\psi_{i}, i \in[1, N]\right.$ with $\left.\psi_{1}=0\right)$ together with the number of teeth of gears were shown to define amplitude modulations due to carrier rotation in Chapter 3 , it is essential that the dynamic model should have the capability to model a gear set with the same parameters as well.

The dynamic model of the planetary gear set shown in Fig. 4.1 allows each gear and carrier body $j$ to translate in $x$ and $y$ directions, denoted by $x_{j}$ and $y_{j}$, and rotate about its axis that remains normal to the transverse plane of the gears. The fluctuations of the same member about its rigid body rotation is defined by $\theta_{j}$. Each planet (pi) is in mesh with the sun gear $(s)$ and the ring (internal) gear $(r)$ while it is supported through its axis by a bearing held by the carrier (c). In Fig. 4.1, only one of the $N$ planet branches located at an arbitrary planet position angle $\psi_{i}$ is shown. In this figure, $\bar{\theta}_{j c}(j=s, r, p i)$ are the nominal kinematic rotations relative to the carrier and $\varepsilon_{j}$ represents the initial position angle of the run-out (eccentricity) vector of each gear $j$. Here, $\varepsilon_{S}$ and $\varepsilon_{r}$ are both defined from the horizontal $x$ axis while $\varepsilon_{p i}$ is defined from the radial axis along the centers of the sun gear $(O)$ and planet pi located at an angle $\psi_{i}$.

Here, dynamic models of a gear pair (external or internal) sub-system and a planet-carrier sub-system shown in Fig. 4.2, will be formulated first before assembling the dynamic model of the entire gear set. Figures 4.2(a) and 4.2(b) show dynamic models 


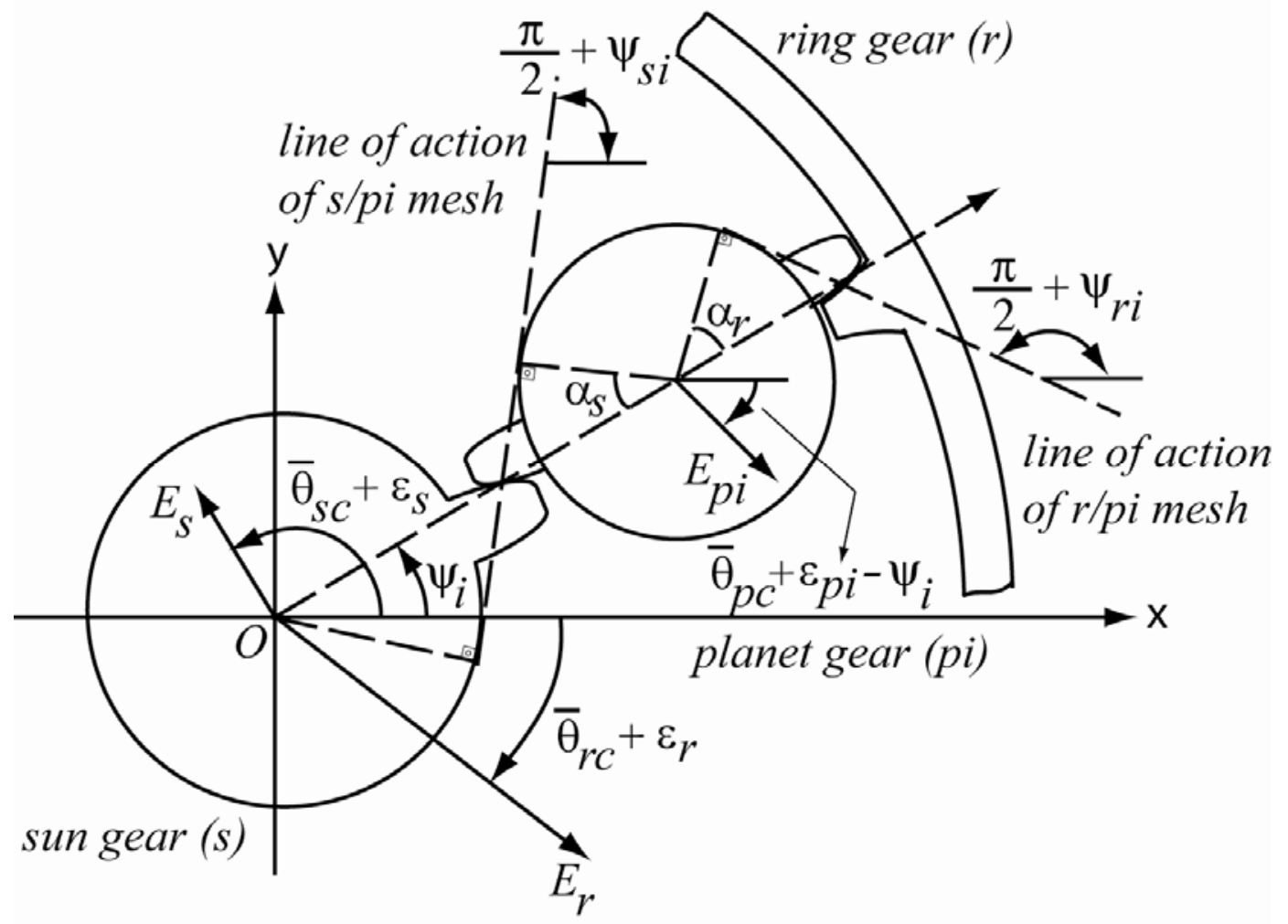

Figure 4.1: Components and basic geometric parameters of a simple planetary gear set (only one planet branch is shown). 

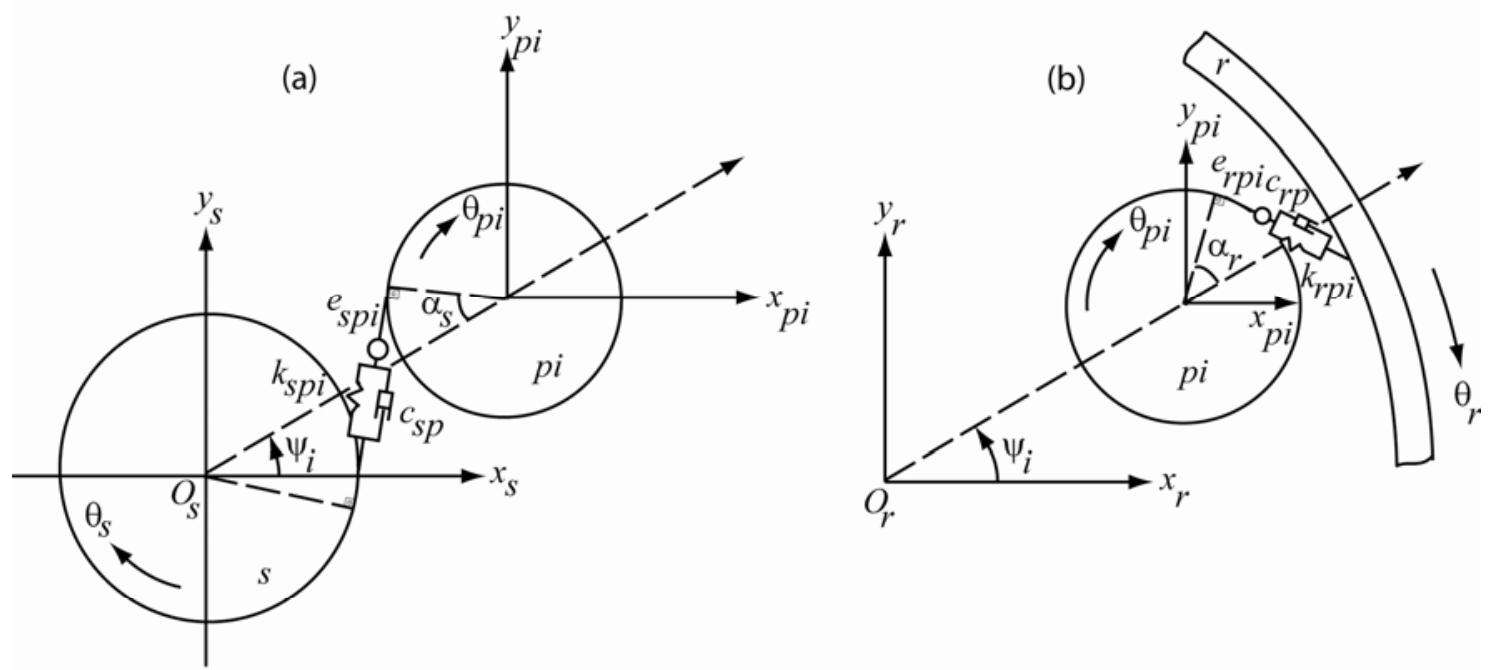

(c)

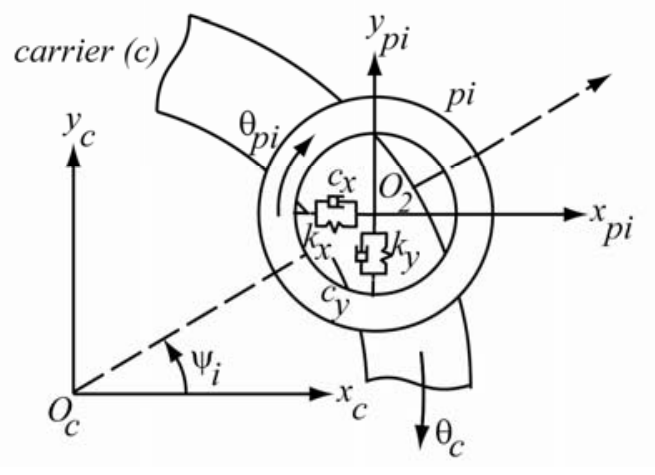

Figure 4.2: Dynamic models of (a) a sun-planet pi pair, (b) a ring-planet pi pair, and (c) a carrier-planet pi pair. 
of external (sun-planet pi) and internal (ring-planet pi) gear pairs, respectively, with planet pi positioned at angle $\psi_{i}$. Gears are modeled as rigid disks with masses $m_{S}, m_{r}$ and $m_{p}$, and polar mass moments of inertia $J_{s}, J_{r}$ and $J_{p}$. The gear mesh interfaces are represented by (i) periodically time-varying gear mesh stiffness elements $k_{s p i}(t)$ and $k_{r p i}(t)$ that account for the fluctuation of the stiffness with gear rotations [4.20], (ii) constant gear mesh damping elements $c_{s p}$ and $c_{r p}$ that are assumed to be the same for each s-pi and r-pi mesh, and (iii) periodic gear transmission error displacement excitations $e_{\text {spi }}(t)$ and $e_{r p i}(t)[4.20]$. Define

$$
u_{s}=r_{s} \theta_{s}, \quad u_{r}=r_{r} \theta_{r}, \quad u_{p i}=r_{p i} \theta_{p i}
$$

as the coordinates in place of $\theta_{s}, \theta_{r}$ and $\theta_{p i}(i \in[1, N])$ where $r_{s}, r_{r}$ and $r_{p i}$ are the radii of the sun, ring and planet gears, respectively. Further, define the dynamic gear mesh force of $j$-pi gear mesh $(j=s, r)$ as

$$
F_{j p i}(t)=c_{j p} \dot{p}_{j p i}(t)+k_{j p i}(t) p_{j p i}(t)
$$

where $p_{j p i}(t)$ is the relative gear mesh displacement

$$
\begin{aligned}
p_{j p i}(t)=\left[y_{j}(t)-\right. & \left.y_{p i}(t)\right] \cos \psi_{j i}-\left[x_{j}(t)-x_{p i}(t)\right] \sin \psi \\
& -u_{j i}(t)-\delta_{j} u_{p i}(t)-e_{j p i}(t)+E_{j p i}(t)-E_{p i j}(t) .
\end{aligned}
$$

With this, equations of motion of a $j$-pi gear pair sub-system are written as 


$$
\begin{aligned}
& m_{j} \ddot{y}_{j}(t)+h_{j p i} \cos \psi_{j i} F_{j p i}(t)=0, \\
& m_{j} \ddot{x}_{j}(t)-h_{j p i} \sin \psi_{j i} F_{j p i}(t)=0, \\
& \frac{J_{j}}{r_{j}^{2}} \ddot{u}_{j}(t)-h_{j p i} F_{j p i}(t)=\frac{T_{j}}{N r_{j}}, \\
& m_{p} \ddot{y}_{p i}(t)-h_{j p i} \cos \psi_{j i} F_{j p i}(t)=0, \\
& m_{p} \ddot{x}_{p i}(t)+h_{j p i} \sin \psi_{j i} F_{j p i}(t)=0, \\
& \frac{J_{p}}{r_{p}^{2}} \ddot{u}_{p i}(t)-\delta_{j} h_{j p i} F_{j p i}(t)=0 .
\end{aligned}
$$

In these equations,

$$
\delta_{j}=\left\{\begin{array}{l}
1 \text { for } j=s, \\
-1 \text { for } j=r
\end{array}\right.
$$

to distinguish between an external s-pi gear pair and an internal $r$-pi gear pair and

$$
\psi_{j i}=\psi_{i}-\delta_{j} \alpha_{j}
$$

as defined in Fig. 4.1 where $\alpha_{j}$ is the transverse pressure angle of the gears. The term $T_{j}$ in Eq. (4.3c) represents the external torque applied on the sun or the ring gear.

An additional function $h_{j p i}$ is applied in Eq. (4.3) as a multiplier to $F_{j p i}(t)$ to account for any nonlinear effects that might take place due to tooth separations. Here, 
$h_{j p i}=1$ when the gear teeth are in contact (i.e. $\left.p_{j p i}(t) \geq 0\right)$ and $h_{j p i}=0$ when gears loose contact (i.e. $\left.p_{j p i}(t)<0\right)$. Also included in Eq. (4.2) are $E_{j p i}(t)$ and $E_{p i j}(t)$ to represent manufacturing errors of gear $j$ relative the planet $p i$ and manufacturing errors of planet $p i$ relative to gear $j$, respectively. These errors will be defined explicitly in the next section. Equations (4.3) can be written in matrix form to obtain sub-matrices associated with the gear $j$ - planet pi pair as

$$
\begin{aligned}
& \mathbf{M}_{j p i}=\left[\begin{array}{cc}
\mathbf{m}_{j} & \mathbf{0} \\
\mathbf{0} & \mathbf{m}_{p i}
\end{array}\right], \\
& \mathbf{C}_{j p i}=\left[\begin{array}{ll}
\mathbf{c}_{j p i}^{(11)} & \mathbf{c}_{j p i}^{(12)} \\
\mathbf{c}_{j p i}^{(12)} & \mathbf{c}_{j p i}^{(22)}
\end{array}\right], \\
& \mathbf{K}_{j p i}=\left[\begin{array}{ll}
\mathbf{k}_{j p i}^{(11)} & \mathbf{k}_{j p i}^{(12)} \\
\mathbf{k}_{j p i}^{(12)} & \mathbf{k}_{j p i}^{(22)}
\end{array}\right],
\end{aligned}
$$

with displacement and force vectors

$$
\begin{aligned}
& \mathbf{Q}_{j p i}=\left\{\begin{array}{c}
\mathbf{q}_{j} \\
\mathbf{q}_{p i}
\end{array}\right\}, \\
& \overline{\mathbf{F}}_{j p i}=\left\{\begin{array}{c}
\overline{\mathbf{f}}_{j} \\
\mathbf{0}
\end{array}\right\},
\end{aligned}
$$




$$
\mathbf{F}_{j p i}(t)=\left\{\begin{array}{c}
\mathbf{f}_{j p i}^{(1)} \\
\mathbf{f}_{j p i}^{(2)}
\end{array}\right\} .
$$

Meanwhile, equations of motion for the carrier-planet pi subsystem shown in Fig. 4.2(c) with $u_{c}=r_{c} \theta_{c}$ as the rotational carrier coordinate $\left(r_{c}\right.$ is the radius of the circle passing through the planet centers) are defined as

$$
\begin{aligned}
& m_{c} \ddot{y}_{c}(t)+c_{y}\left[\dot{y}_{c}(t)-\dot{y}_{p i}(t)-\cos \psi_{i} \dot{u}_{c}(t)\right] \\
& +k_{y}\left[y_{c}(t)-y_{p i}(t)-\cos \psi_{i} u_{c}(t)\right]=0, \\
& m_{C} \ddot{x}_{C}(t)+c_{X}\left[\dot{x}_{C}(t)-\dot{x}_{p i}(t)+\sin \psi_{i} \dot{u}_{C}(t)\right] \\
& +k_{X}\left[x_{c}(t)-x_{p i}(t)+\sin \psi_{i} u_{c}(t)\right]=0, \\
& \frac{J_{C}}{r_{C}^{2}} \ddot{u}_{C}(t)+c_{x}\left[\dot{x}_{C}(t)-\dot{x}_{p i}(t)+\sin \psi_{i} \dot{u}_{C}(t)\right] \sin \psi_{i} \\
& -c_{y}\left[\dot{y}_{c}(t)-\dot{y}_{p i}(t)-\cos \psi_{i} \dot{u}_{c}(t)\right] \cos \psi_{i} \\
& +k_{x}\left[x_{c}(t)-x_{p i}(t)+\sin \psi_{i} u_{c}(t)\right] \sin \psi_{i} \\
& -k_{y}\left[y_{C}(t)-y_{p i}(t)-\cos \psi_{i} u_{C}(t)\right] \cos \psi_{i}=\frac{T_{C}}{N r_{C}}, \\
& m_{p} \ddot{y}_{p i}(t)-c_{y}\left[\dot{y}_{c}(t)-\dot{y}_{p i}(t)-\cos \psi_{i} \dot{u}_{c}(t)\right] \\
& -k_{y}\left[y_{c}(t)-y_{p i}(t)-\cos \psi_{i} u_{c}(t)\right]=0, \\
& m_{p} \ddot{x}_{p i}(t)-c_{X}\left[\dot{x}_{c}(t)-\dot{x}_{p i}(t)+\sin \psi_{i} \dot{u}_{c}(t)\right] \\
& -k_{x}\left[x_{C}(t)-x_{p i}(t)+\sin \psi_{i} u_{C}(t)\right]=0 .
\end{aligned}
$$


Here, $T_{C}$ is the external torque applied to the carrier, and $m_{C}$ and $J_{C}$ are the mass and the mass moment of inertia of the carrier, respectively. In this model, the planet pin-bearing assembly is represented by linear springs $k_{x}$ and $k_{y}$, and dampers $c_{x}$ and $c_{y}$ as illustrated in Fig. 4.2(c). It is noted that above equations are not a function of planet rotation $u_{p i}=r_{p} \theta_{p i}$ since planet $p i$ can rotate freely relative to the carrier without exerting any force. Equations (4.6) are written in matrix form to define

$$
\begin{aligned}
& \mathbf{M}_{c p i}=\left[\begin{array}{cc}
\mathbf{m}_{c} & \mathbf{0} \\
\mathbf{0} & \mathbf{m}_{p i}
\end{array}\right], \\
& \mathbf{C}_{p i}=\left[\begin{array}{ll}
\mathbf{c}_{c p i}^{(11)} & \mathbf{c}_{c p i}^{(12)} \\
\mathbf{c}_{c p i}^{(12)} & \mathbf{c}_{c p i}^{(22)}
\end{array}\right], \\
& \mathbf{K}_{c p i}=\left[\begin{array}{ll}
\mathbf{k}_{c p i}^{(11)} & \mathbf{k}_{c p i}^{(12)} \\
\mathbf{k}_{c p i}^{(12)} & \mathbf{k}_{c p i}^{(22)}
\end{array}\right], \\
& \mathbf{Q}_{c p i}=\left\{\begin{array}{c}
\mathbf{q}_{c} \\
\mathbf{q}_{p i}
\end{array}\right\}, \\
& \overline{\mathbf{F}}_{c p i}=\left\{\begin{array}{l}
\overline{\mathbf{f}}_{c} \\
\mathbf{0}
\end{array}\right\} .
\end{aligned}
$$

With the matrices of every sub-system available, the overall equations of motion of an $N$-planet planetary gear set with planets spaced at $\psi_{i}(i \in[1, N])$ are constructed systematically as 


$$
\mathbf{M} \ddot{\mathbf{Q}}(t)+\left[\mathbf{C}+\mathbf{C}_{b}\right] \dot{\mathbf{Q}}(t)+\left[\mathbf{K}(t)+\mathbf{K}_{b}\right] \mathbf{Q}(t)=\overline{\mathbf{F}}+\mathbf{F}(t),
$$

where

$$
\mathbf{Q}=\left[\begin{array}{llllll}
\mathbf{q}_{c} & \mathbf{q}_{r} & \mathbf{q}_{s} & \mathbf{q}_{p 1} & \ldots & \mathbf{q}_{p N}
\end{array}\right]^{T}
$$

$$
\mathbf{M}=\operatorname{Diag}\left[\begin{array}{llllll}
\mathbf{M}_{c} & \mathbf{M}_{r} & \mathbf{M}_{s} & \mathbf{M}_{p 1} & \ldots & \mathbf{M}_{p N}
\end{array}\right] .
$$

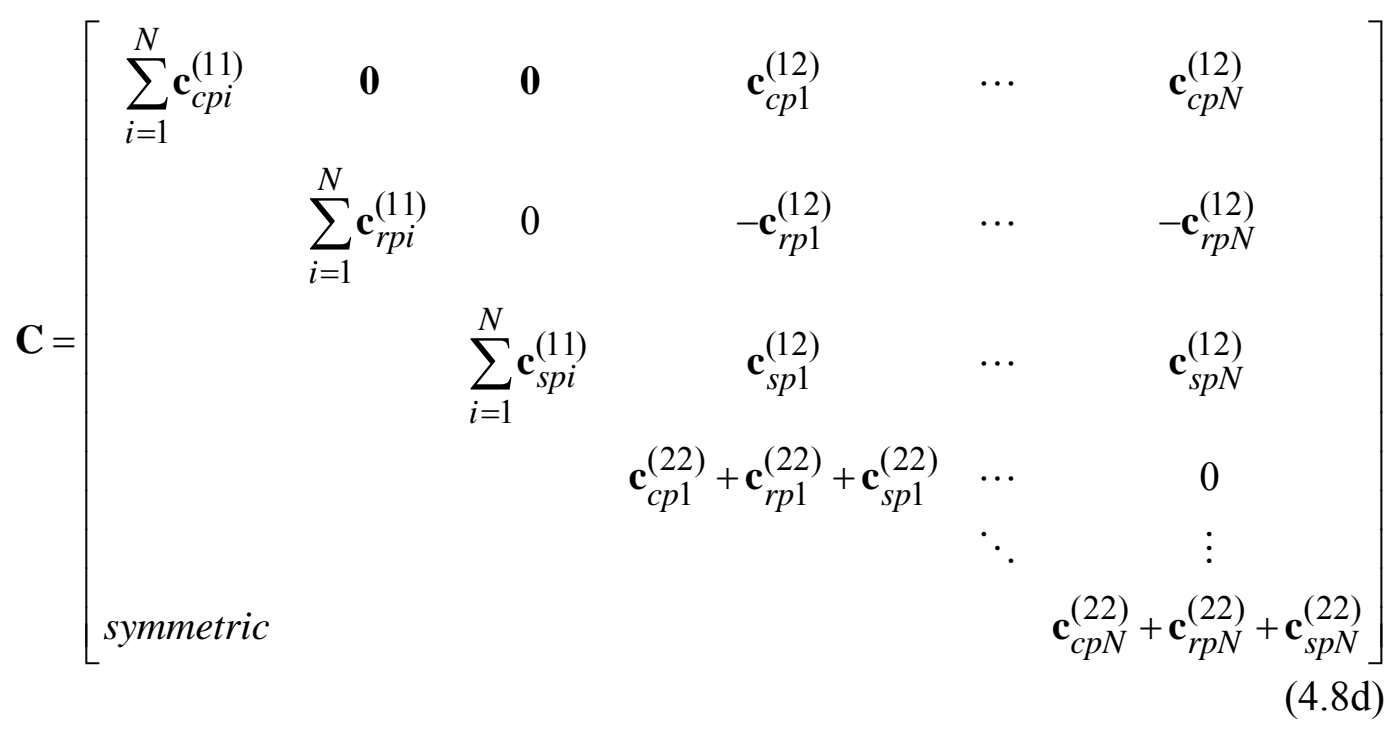

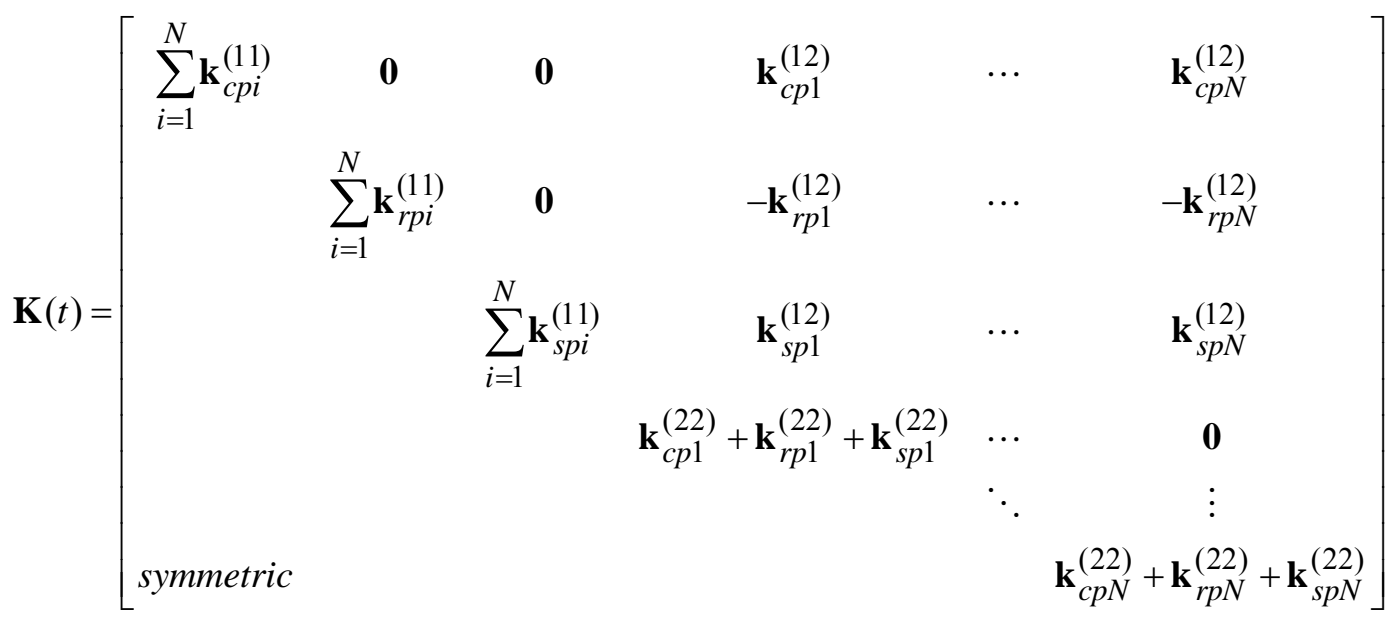


In Eq. (4.8e), the elements of $\mathbf{K}(t)$ are periodically time-varying since they are functions of time-varying mesh stiffnesses $k_{s p i}(t)$ and $k_{r p i}(t)(i \in[1, N])$.

The $\mathbf{C}_{b}$ and $\mathbf{K}_{b}$ matrices in Eq. (4.8a) represent the bearing damping and stiffness matrices that are assumed to be diagonal matrices as shown below $(j=s, r, c)$ :

$$
\begin{aligned}
\mathbf{C}_{b} & =\operatorname{Diag}\left[\begin{array}{llllll}
\mathbf{c}_{b c} & \mathbf{c}_{b r} & \mathbf{c}_{b s} & \mathbf{0} & \cdots & \mathbf{0}
\end{array}\right], \\
\mathbf{K}_{b} & =\operatorname{Diag}\left[\begin{array}{llllll}
\mathbf{k}_{b c} & \mathbf{k}_{b r} & \mathbf{k}_{b s} & \mathbf{0} & \cdots & \mathbf{0}
\end{array}\right] .
\end{aligned}
$$

Here, sub-matrices $\mathbf{c}_{b j}=\operatorname{Diag}\left[\begin{array}{lll}c_{x j} & c_{y j} & c_{u j}\end{array}\right]$ and $\mathbf{k}_{b j}=\operatorname{Diag}\left[\begin{array}{lll}k_{x j} & k_{y j} & k_{u j}\end{array}\right]$ are the diagonal damping and stiffness matrices of the bearing supporting central member $j$ $(j=s, r, c)$. If any central member $j$ is allowed to float radially (i.e. not supported externally), then $k_{x j}=k_{y j} \approx 0$. If a central member is held stationary, then $k_{u j}$ has a large value representing the stiffness of the holding structure.

The forcing term at the right hand side of Eq. (4.8a) consists of a mean force vector $\overline{\mathbf{F}}$ and an alternating (time-varying) force vector $\mathbf{F}(t)$, which are defined as

$$
\overline{\mathbf{F}}=N\left\{\begin{array}{c}
\overline{\mathbf{f}} \\
\overline{\mathbf{f}}_{r} \\
\overline{\mathbf{f}}_{s} \\
\mathbf{0} \\
\vdots \\
\mathbf{0}
\end{array}\right\},
$$




$$
\mathbf{F}(t)=\left\{\begin{array}{c}
\mathbf{0} \\
\sum_{i=1}^{N} \mathbf{f}_{r p i}^{(1)} \\
\sum_{i=1}^{N} \mathbf{f}_{s p i}^{(1)} \\
\mathbf{f}_{s p 1}^{(2)}-\mathbf{f}_{r p 1}^{(2)} \\
\vdots \\
\mathbf{f}_{s p N}^{(2)}-\mathbf{f}_{r p N}^{(2)}
\end{array}\right\} .
$$

Here $\mathbf{F}(t)$ contains all of the external displacement excitations $e_{s p i}(t)$ and $e_{r p i}(t)$ at the gear mesh frequency as well as the excitations $E_{s p i}(t), E_{p i s}(t), E_{r p i}(t)$ and $E_{p i r}(t)$ at the relative gear rotational frequencies.

\subsubsection{Formulation of Modulations of the Excitations Due to Manufacturing Errors}

The error functions $E_{s p i}(t), E_{p i s}(t), E_{r p i}(t)$ and $E_{p i r}(t)$ given in Eq. (4.2b) as part of the relative gear mesh displacements represent errors associated with rotational frequencies of gears relative to the carrier. Such errors include gear eccentricities, pitch line run-out errors, tooth spacing and indexing errors, all of which are approximated to harmonic forms for both the s-pi and $r$-pi meshes as

$$
\begin{aligned}
& E_{s p i}(t)=E_{s} \sin \left[\frac{\omega_{m}}{Z_{s}} t+\varepsilon_{s}-\psi_{s i}\right], \\
& E_{p i s}(t)=E_{p i} \sin \left[\frac{\omega_{m}}{Z_{p}} t+\varepsilon_{p i}-\alpha_{s}\right],
\end{aligned}
$$




$$
\begin{aligned}
& E_{r p i}(t)=E_{r} \sin \left[\frac{\omega_{m}}{Z_{r}} t+\varepsilon_{r}+\psi_{r i}\right], \\
& E_{p i r}(t)=E_{p i} \sin \left[\frac{\omega_{m}}{Z_{p}} t+\varepsilon_{p i}+\alpha_{r}\right] .
\end{aligned}
$$

Here, $\omega_{m}$ is the gear mesh frequency that is defined as

$$
\omega_{m}=Z_{s}\left|\omega_{s}-\omega_{c}\right|=Z_{r}\left|\omega_{r}-\omega_{c}\right|=Z_{p}\left|\omega_{p}-\omega_{c}\right|
$$

where $\omega_{j}$ and $Z_{j}$ are the absolute nominal angular velocity and number of teeth of gear $j \quad(j=r, s, p)$ and $\omega_{c}$ is the absolute nominal angular velocity of the carrier. Accordingly, the excitations $E_{\text {pis }}(t)$ and $E_{\text {pir }}(t)$ of pinion pi are at a frequency $\omega_{m} / Z_{p}$ that is equal to the rotational velocity of $p i$ relative to $c$. Similarly $E_{s p i}(t)$ and $E_{r p i}(t)$ are at frequencies $\omega_{m} / Z_{s}$ and $\omega_{m} / Z_{r}$, respectively, representing the rotational velocities of the sun and ring gears relative to the carrier. Coefficients $E_{S}, E_{r}$ and $E_{p i}$ are the amplitudes of these errors. Additional phase angles $\varepsilon_{S}, \varepsilon_{r}$ and $\varepsilon_{p i}$, as illustrated in Fig. 4.1, define the initial orientation of them as previously defined.

In the absence of errors defined in Eq. (4.11), gear transmission error excitations defined as a part of the gear mesh interface in Fig. 4.2(a,b) are given in periodic form as [4.20]

$$
e_{s p i}(t)=\sum_{\ell=1}^{L} e_{s p}^{(\ell)} \sin \left[\ell \omega_{m} t+\ell Z_{s} \psi_{i}+\phi_{s p}^{(\ell)}\right]
$$




$$
e_{r p i}(t)=\sum_{\ell=1}^{L} e_{r p}^{(\ell)} \sin \left[\ell \omega_{m} t+\ell Z_{r} \psi_{i}+\ell \gamma_{s r}+\phi_{r p}^{(\ell)}\right]
$$

where $\gamma_{s r}$ is the phase angle between $e_{s p i}(t)$ and $e_{r p i}(t)$.

If the dynamic model proposed in the previous section were to be exercised with excitations as defined in Eq. (4.11) and (4.13) as it was done in Ref. [4.20], one would observe little or no modulation sidebands in dynamic gear mesh forces $F_{s p i}(t)$ and $F_{r p i}(t)$ as there is no interaction between the two groups of excitations, especially in linear regimes. In reality, however, the presence of errors as in Eq. (4.11) causes $e_{s p i}(t)$ and $e_{r p i}(t)$ to be amplitude and phase modulated, forming the basis for sidebands due to manufacturing errors of gears. In presence of errors defined in Eq. (4.11), a quasi-static deformable body contact analysis of a typical automotive external gear pair using a gear load distribution model [4.21] shows that the peak-to-peak amplitude of $e_{\text {spi }}(t)$ might experience up to 4 to $6 \%$ variation due to $E_{\text {spi }}(t)$ and $E_{p i s}(t)$ even with modest error magnitudes of $E_{S}$ or $E_{p i}$ (say $20 \mu \mathrm{m}$ ) depending on the instantaneous direction of the error of one gear with respect to the mating gear. In addition to this apparent amplitude modulation, a frequency modulation is also resulted since the variation in effective center distance caused by these errors modulates the mesh period. These indicate that Eq. (4.13) must be modified in the presence of the gear errors defined in Eq. (4.11).

In order to show the impact of $E_{s p i}(t), E_{p i s}(t), E_{r p i}(t)$ and $E_{p i r}(t)$ on $e_{s p i}(t)$ and $e_{r p i}(t)$, consider only the fundamental harmonic of $e_{s p i}(t)$ and $e_{r p i}(t)$ (i.e. $L=1$ in 
Eq. (4.13) with $e_{s p}^{(1)}=e_{s p}, \phi_{s p}^{(1)}=\phi_{s p}, e_{r p}^{(1)}=e_{r p}$ and $\left.\phi_{r p}^{(1)}=\phi_{r p}\right)$. The excitations $e_{s p i}(t)$ and $e_{r p i}(t)$ with amplitude and frequency modulations can be written as

$$
\begin{aligned}
& e_{s p i}(t)=A_{s p i}(t) e_{s p} \sin \left[B_{s p i}(t) \omega_{m} t+Z_{s} \psi_{i}+\phi_{s p}\right], \\
& A_{s p i}(t)=1+\beta_{s} \sin \left(\frac{\omega_{m}}{Z_{s}} t+\phi_{\beta s i}\right)+\beta_{p i} \sin \left(\frac{\omega_{m}}{Z_{p}} t+\phi_{\beta p i}\right), \\
& B_{s p i}(t)=1+\hat{\beta}_{s} \sin \left[\frac{\omega_{m}}{Z_{s}} t+\hat{\phi}_{\hat{\beta} s i}\right]+\hat{\beta}_{p i} \sin \left[\frac{\omega_{m}}{Z_{p}} t+\hat{\phi}_{\hat{\beta} p i}\right],
\end{aligned}
$$

and

$$
\begin{aligned}
& e_{r p i}(t)=A_{r p i}(t) e_{r p} \sin \left[B_{r p i}(t) \omega_{m} t+Z_{r} \psi_{i}+\gamma_{s r}+\phi_{r p}\right], \\
& A_{r p i}(t)=1+\beta_{r} \sin \left[\frac{\omega_{m}}{Z_{s}} t+\phi_{\beta r i}\right]+\vartheta_{p i} \sin \left[\frac{\omega_{m}}{Z_{p}} t+\phi_{\vartheta p i}+\pi\right], \\
& B_{r p i}(t)=1+\hat{\beta}_{r} \sin \left[\frac{\omega_{m}}{Z_{s}} t+\hat{\phi}_{\hat{\beta} r}\right]+\hat{\vartheta}_{p i} \sin \left[\frac{\omega_{m}}{Z_{p}} t+\hat{\phi}_{\hat{\vartheta} p i}+\pi\right] .
\end{aligned}
$$

Here, dimensionless coefficients $\beta_{s}$ and $\beta_{p i}$ represent the amplitude modulation of the fundamental harmonic of $e_{s p i}(t)$ due the errors of the sun gear and planet pi, and $\phi_{\beta s i}$ and $\phi_{\beta p i}$ are the phase angles for the same gears, as defined by the initial position angles $\varepsilon_{s}$ and $\varepsilon_{p i}$. Parameters $\beta_{r}$ and $\phi_{\beta r i}$ define the amplitude and the phase angle of the 
amplitude modulation of $e_{r p i}(t)$ due to the error of the ring gear. Parameters $\vartheta_{p i}$ and $\phi_{\vartheta p i}$ define the amplitude and the phase angle of the amplitude modulation of $e_{r p i}(t)$ due the error of the planet gear. Here, it is important to note that the amplitude modulation coefficients $\left(\beta_{p i}\right.$ and $\left.\vartheta_{p i}\right)$ are usually different for the $s-p i$ and $r$-pi meshes involving the same planet pi for the same planet run-out amount. Similarly, amplitude coefficients, $\hat{\beta}_{s}, \hat{\beta}_{r}, \hat{\beta}_{p i}$ and $\hat{\vartheta}_{p i}$ and the phase angles $\hat{\phi}_{\hat{\beta} s i}, \hat{\phi}_{\hat{\beta} r i}$, $\hat{\phi}_{\hat{\beta} p i}$ and $\hat{\phi}_{\hat{\vartheta} p i}$ define the frequency modulations of $e_{s p i}(t)$ and $e_{r p i}(t)$. All these modulation parameters can be defined up-front by simulating the loaded gear contacts under quasi-static conditions [4.21]. In Eq. (4.14) and (4.15), the gear mesh-frequency excitations $e_{s p i}(t)$ and $e_{r p i}(t)$ are both amplitude and frequency modulated by errors at rotational frequencies of gear $s, r$ and $p i$ relative to the planet carrier. These rotational frequencies $\omega_{m} / Z_{s}, \omega_{m} / Z_{r}$ and $\omega_{m} / Z_{p}$ are typically distinct so that sidebands caused by each on the resultant $F_{s p i}(t)$ and $F_{s p i}(t)$ spectra should correspond to a different set of frequencies, as it will be demonstrated later.

The same modulation mechanism affects the periodically time-varying gear mesh stiffness functions of the s-pi and r-pi meshes in the same way. In their unmodulated form, these functions are defined below as

$$
k_{s p i}(t)=\bar{k}_{s p}\left\{1+\sum_{\ell=1}^{L} k_{s p}^{(\ell)} \sin \left[\ell \omega_{m} t+\ell Z_{s} \psi_{i}+\ell \Gamma+\phi_{s p}^{(\ell)}\right]\right\},
$$




$$
k_{r p i}(t)=\bar{k}_{r p}\left\{1+\sum_{\ell=1}^{L} k_{r p}^{(\ell)} \sin \left[\ell \omega_{m} t+\ell Z_{r} \psi_{i}+\ell \Gamma+\ell \gamma_{s r}+\phi_{r p}^{(\ell)}\right]\right\} .
$$

In these equations, $\bar{k}_{s p}$ and $\bar{k}_{r p}$ are the mean mesh stiffness values computed at a given torque value, $k_{s p}^{(\ell)}$ and $k_{r p}^{(\ell)}$ are the dimensionless $\ell$-th harmonic amplitudes, and $\Gamma$ is the phase angle between $e_{s p i}(t)$ and $k_{s p i}(t)$ (and also between $e_{r p i}(t)$ and $\left.k_{r p i}(t)\right)$. Considering only the fundamental harmonic terms $(L=1)$ for demonstration purposes and modulating the amplitudes and the mesh frequency the same way as in Eq. (4.14) and (4.15), one obtains

$$
\begin{aligned}
& k_{s p i}(t)=\bar{k}_{s p}+C_{s p i}(t) k_{s p} \sin \left[D_{s p i}(t) \omega_{m} t+Z_{s} \psi_{i}+\Gamma+\phi_{s p}\right], \\
& C_{s p i}(t)=1+\kappa_{s} \sin \left[\frac{\omega_{m}}{Z_{s}} t+\phi_{\kappa s i}\right]+\kappa_{p i} \sin \left[\frac{\omega_{m}}{Z_{p}} t+\phi_{\kappa p i}\right], \\
& D_{s p i}(t)=1+\hat{\kappa}_{s} \sin \left[\frac{\omega_{m}}{Z_{s}} t+\hat{\phi}_{\hat{\kappa} s i}\right]+\hat{\kappa}_{p i} \sin \left[\frac{\omega_{m}}{Z_{p}} t+\hat{\phi}_{\hat{\kappa} p i}\right],
\end{aligned}
$$

and

$$
\begin{aligned}
& k_{r p i}(t)=\bar{k}_{r p}+C_{r p i}(t) k_{r p} \sin \left[D_{r p i}(t) \omega_{m} t+Z_{r} \psi_{i}+\gamma_{s r}+\Gamma+\phi_{r p}\right], \\
& C_{r p i}(t)=1+\kappa_{r} \sin \left[\frac{\omega_{m}}{Z_{r}} t+\phi_{\kappa r i}\right]+\tau_{p i} \sin \left[\frac{\omega_{m}}{Z_{p}} t+\phi_{\tau p i}+\pi\right],
\end{aligned}
$$




$$
D_{r p i}(t)=1+\hat{\kappa}_{r} \sin \left[\frac{\omega_{m}}{Z_{r}} t+\hat{\phi}_{\hat{\kappa} r i}\right]+\hat{\tau}_{p i} \sin \left[\frac{\omega_{m}}{Z_{p}} t+\hat{\phi}_{\hat{\tau} p i}+\pi\right] .
$$

\subsubsection{Formulation of Modulations due to Rotating Carrier}

With the modulated excitations defined by Eq. (4.14) to (4.18), the equations of motion (4.8a) of the nonlinear time-varying planetary gear system are solved by using direct numerical integration technique to determine the unknown vector of displacement time histories $\mathbf{Q}(t)$, from which the dynamic gear mesh forces $F_{s p i}(t)$ and $F_{r p i}(t)$ can be computed as defined in Sect. 4.2.1. As it will be demonstrated in the next section, these gear mesh forces exhibit sideband activity associated with the once-per-revolution errors of the gears. If the power flow configuration is such that the carrier is held stationary (i.e. sun and ring gears serve as input and output members), then any vibration or noise spectra measured in a fixed location in the vicinity of the gear set will have the same sideband structure. As described in detail in Chapter 3, another layer of modulations take place when the carrier is rotating, as the influence of gear mesh forces (now rotating with the carrier) are amplitude modulated, as viewed from a fixed measurement point. The dynamic mesh forces $F_{s p i}(t)$ and $F_{r p i}(t)$ predicted by the proposed dynamic model with or without the other gear errors can be used as input for the model of Chapter 3 to predict the spectral content for the rotating carrier case.

Consider the same planetary gear set with $N$ planets positioned at angles $\psi_{i}$ $(i \in[1, N])$ and with rotating carrier $c$ and the sun gear $s$ (fixed: ring gear $r$ ). For a 
complete revolution of the carrier, a transducer positioned on the housing shown in Figure 3.1 experiences the disturbances from all $2 \mathrm{~N}$ planet meshes in sequence. As the force transmission path between the meshes of planet $p i$ and the fixed transducer location varies in time as the carrier rotates, the influence of each planet on the transducer was limited in Chapter 3 to a duration of $T_{C} / N$ where $T_{C}=2 \pi / \omega_{C}$ is the rotational period of the carrier. This gradually increasing and then decaying dominance of the forces of planet $p i$ within a $T_{C} / N$ time period, was approximated by weighting function in the form

$$
w_{i}(t)=w\left(t-\frac{\psi_{i}}{2 \pi} T_{C}\right) \sum_{n=1}^{\infty}\left\{u\left[t-\left(\frac{(n-1) N+i-1}{N}\right) T_{C}\right]-u\left[t-\left(\frac{(n-1) N+i}{N}\right) T_{C}\right]\right\} .
$$

where $\psi_{i}$ is the position angle of planet pi. In this equation, terms $u(t-a)$ are unit step functions $(u(t-a)=1$ for $t>a$ and $u(t-a)=0$ for $t<a)$ that ensure the influence of planet $p i$ on the transducer lasts only for a period of $T_{C} / N$. The summation over $n$ ensures the periodicity of the function.

The acceleration signal at the transducer location caused by dynamic forces $F_{s p i}(t)$ and $F_{r p i}(t)$ of the meshes of planet pi mesh are then defined as

$$
a_{i}(t)=S_{s} w_{i}(t) F_{s p i}(t)+S_{r} w_{i}(t) F_{r p i}(t)
$$


where $S_{S}$ and $S_{r}$ are constants devised to establish the relation between the gear mesh forces and the resultant acceleration at a given rotational position. The total acceleration signal $a(t)$ that is given as

$$
a(t)=\sum_{i=1}^{N} a_{i}(t)
$$

with a frequency spectrum

$$
A(\omega)=\left|\int_{-\infty}^{\infty} a(t) e^{-i \omega t} d t\right|
$$

For the case of non-rotating carrier, all $w_{i}(t)=1$ with different $S_{s}$ are $S_{r}$ values for each planet depending on their distance from the transducer location.

\subsection{An Example Analysis of Modulation Sidebands of a Planetary Gear Set with Manufacturing Errors}

In this section, an example planetary gear set design is used to demonstrate different modulation mechanisms for systems with and without a rotating carrier under different dynamic conditions. Here, a 4-planet planetary gear set is chosen as the example system having gears with tooth numbers $Z_{r}=140, Z_{s}=80$ and $Z_{p}=30$. Gear mesh parameters of $\bar{k}_{s p}=5.2(10)^{8} \mathrm{~N} / \mathrm{m}, \quad k_{s p}^{(1)}=17.5(10)^{6} \mathrm{~N} / \mathrm{m}, \bar{k}_{r p}=5.5(10)^{8} \mathrm{~N} / \mathrm{m}$, $k_{r p}^{(1)}=18.6(10)^{6} \mathrm{~N} / \mathrm{m}, \quad e_{s p}^{(1)}=0.5 \mu \mathrm{m}$ and $e_{r p}^{(1)}=0.16 \mu \mathrm{m}$ are used for harmonic forms of 
the mesh stiffness and transmission error excitations. Other parameters of this gear set that are relevant to the dynamic model are listed in Table 4.1.

Here, the planets are equally spaced $\left(\psi_{i}=2 \pi(i-1) / 4\right)$ and planet meshes are inphase type $\left(Z_{S} / N=\right.$ integer and $Z_{r} / N=$ integer $)$ such that this gear set falls into the Case (i) category according to the classification of planetary gear sets proposed in Chapter 3 based on their sideband behavior. Planetary gear sets in this category were shown to have a relatively simple sideband activity due to the carrier rotation. This will allow a more clear demonstration of the sidebands due to manufacturing errors with limited added complexity due to carrier rotation, while the model is capable of handling any of five categories defined in Chapter 3. For the same reason, AM and FM modulations will be introduced separately with one gear error at a time. Both dynamic gear mesh force spectra and the vibration spectra at a fixed point will be predicted to identify the impact of the rotating carrier. Finally, simulations will be performed at different speeds (frequencies) representing off-resonance and near resonance conditions to highlight the impact of dynamic behavior on the planetary side band activity.

\subsubsection{Planetary Gear Set Modulations under Off-resonance Conditions}

The undamped natural modes of the corresponding linear time-invariant system can be determined by setting $\mathbf{C}=\mathbf{0}$ and $\mathbf{C}_{b}=\mathbf{0}$ in Eq. (4.8a), considering constant gear

mesh stiffnesses with $k_{s p i}(t)=\bar{k}_{s p}$ and $k_{r p i}(t)=\bar{k}_{r p}$ in Eq. (4.17a) and (4.18a), and solving the corresponding eigenvalue problem. 


\begin{tabular}{lccc}
\hline parameter & sun & planet & ring \\
& & & \\
\hline Number of teeth & 80 & 30 & 120 \\
Module, mm & 1.8 & 1.8 & 1.8 \\
Pressure angle, deg & 22.5 & 22.5 & 22.5 \\
Face width, mm & 30 & 30 & 30 \\
Root diameter, mm & 131 & 43.4 & 237.2 \\
Outside diameter, mm & 139.2 & 50 & 229.6 \\
Pin circle diameter, mm & & 78 & \\
Tooth thickness, mm & 2.83 & 2.85 & 2.97 \\
Operating center dis., mm & & 92.1 & \\
\end{tabular}

Table 4.1: Basic design parameters of the example planetary gear set. 
With the numerical values of the parameters defined above and in Table 4.1, first five natural frequencies of this example gear set are determined as $982,2484,2629,5088$ and $5101 \mathrm{~Hz}$, excluding the rigid-body mode at $0 \mathrm{~Hz}$. First, the example planetary gear set is simulated at a lower mesh frequency of $400 \mathrm{~Hz}\left(\omega_{s}=49.4 \mathrm{rad} / \mathrm{s}\right.$ resulting in $\omega_{m}=2513.3 \mathrm{rad} / \mathrm{s}$ in this kinematic configuration with a stationary ring gear) such that a typical off-resonance condition exists. All of the analyses are carried out at a sun torque value of $T_{s}=500 \mathrm{Nm}$.

\section{$\underline{\text { Amplitude Modulations }}$}

First, a planet run-out error of $E_{p 1}=20 \mu \mathrm{m}$ at $\left(\varepsilon_{p 1}=0\right)$ is applied to planet $p 1$ while the other $E_{p i}=0$ and $E_{S}=E_{r}=0$. The corresponding amplitude modulation parameters in Eq. (4.14), (4.15), (4.17) and (4.18) are defined using the gear load distribution model [4.21] as $\beta_{p 1}=0.034, \phi_{\beta p 1}=3 \pi / 2, \vartheta_{p 1}=0.033, \phi_{\vartheta p 1}=\pi / 2$, $\kappa_{p 1}=0.055, \quad \phi_{\kappa p 1}=3 \pi / 2, \tau_{p 1}=0.049$ and $\phi_{\tau p 1}=\pi / 2$ with all other modulation coefficients are set to zero. Predicted dynamic mesh force spectra $F_{s p 1}(\omega)$ and $F_{r p 1}(\omega)$ at s-p1 and $r-p 1$ meshes are shown in Fig. 4.3. In these figures, the $x$ axis represents the output side gear mesh orders obtained by normalizing the frequency scale by the rotational output frequency. With this, the fundamental gear mesh order is $H_{m}=Z_{r}=140$. Two almost symmetric sidebands are evident at both sides of $H_{m}$ at orders of $H_{m} \pm H_{p / c}$ where $H_{p / c}=Z_{r} / Z_{p}=4.69$. Dynamic mesh force spectra for the 

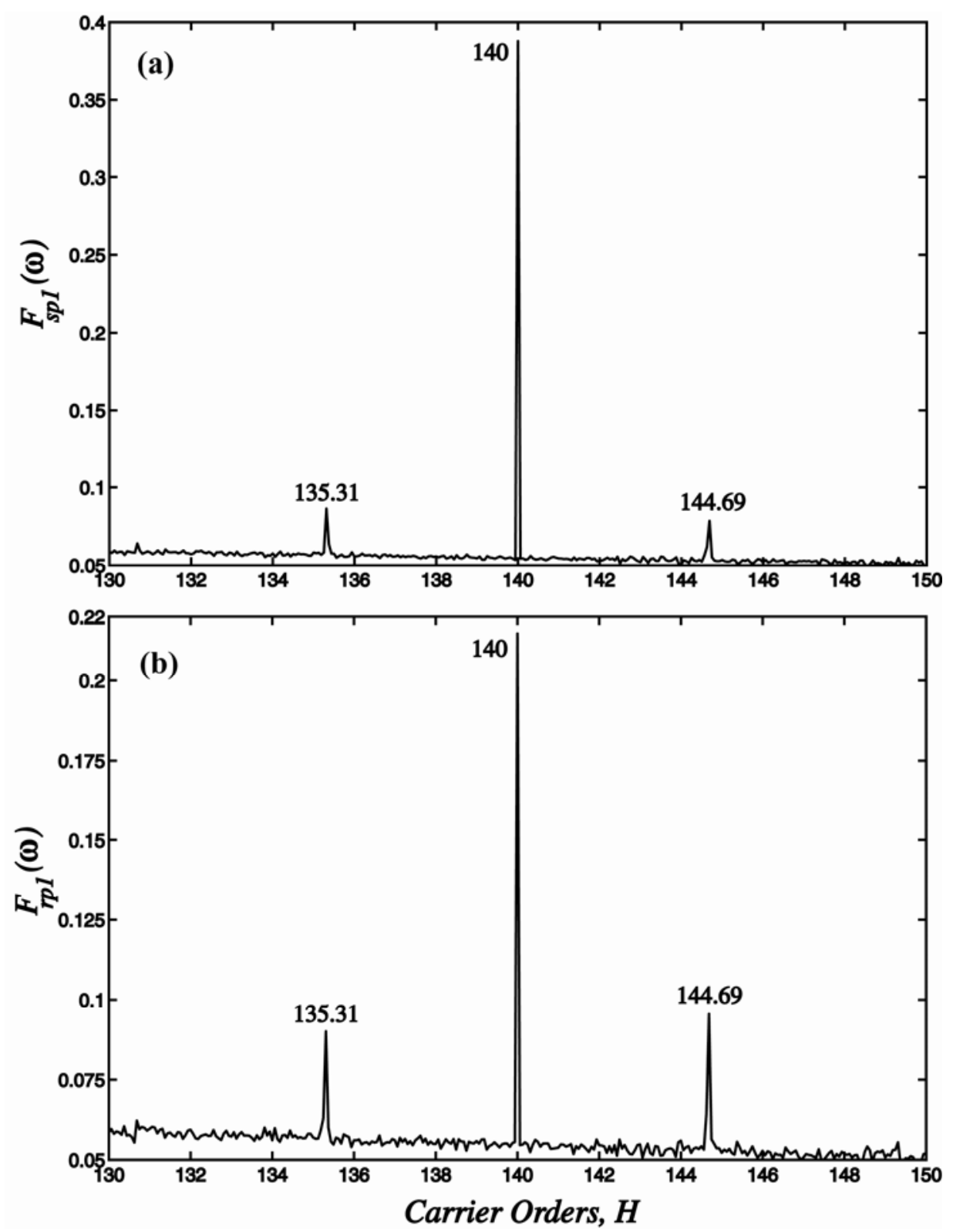

Figure 4.3: Predicted (a) $F_{s p 1}(\omega)$ and (b) $F_{r p 1}(\omega)$ spectra for the example planetary gear set under the influence of AM due to planet-1 run-out error with $\beta_{p 1}=0.034$, $\phi_{\beta p 1}=3 \pi / 2, \vartheta_{p 1}=0.033, \phi_{\vartheta p 1}=\pi / 2, \kappa_{p 1}=0.055, \phi_{\kappa p 1}=3 \pi / 2, \tau_{p 1}=0.049$ and $\phi_{\tau p 1}=\pi / 2$. 
meshes of planets $p 2$ to $p 4$ are also very similar to those shown in Fig. 4.3 for the meshes of the first planet.

With these gear mesh forces, $F_{s p i}(t)$ and $F_{r p i}(t)(i \in[1, N])$, predicted with the sidebands associated with $E_{p 1}$, the procedure outlined in Sect. 4.2.3 is applied next to predict the acceleration spectra measured on the stationary ring gear as illustrated in Fig. 3.1. Figure 4.4 shows the acceleration spectrum $A(\omega)$ for this case, given $S_{S}=0$ and $S_{r}=1$ in Eq. (4.20). Here, some of the energy of the gear mesh harmonic order of $H=H_{m}=140$ is distributed to two sidebands at $H=H_{m} \pm N=140 \pm 4$ as a direct result of the amplitude modulation caused by the rotating carrier. As the run-out (or eccentricity) vector rotates with the planet gear, it has a different phase angle each time the planet enters its windowed region. This causes the force sidebands at orders $H_{m} \pm H_{p / c}$ of Fig. 4.3 (135.31 and 144.69) to cause new sidebands at $H_{m} \pm H_{p / c} \pm n$ ( $n=$ integer) while orders $H_{m} \pm H_{p / c}$ disappear. Among them, the sideband orders $H_{m} \pm H_{p / c} \pm 2(H=133.31,137.31,142.69$ and 146.69) are especially large in addition to those at $H_{m} \pm H_{p / c} \pm 2 \pm N$ (e.g. $H=129.31,141.31,138.69$ and 150.69).

Next, an error amplitude of $E_{S}=20 \mu \mathrm{m}$ is applied to the sun gear of the same gear set while all $E_{p i}=0$ and $E_{r}=0$. Considering only the amplitude modulations in Eq. (4.14), (4.15), (4.17) and (4.18), the dynamic mesh forces are amplitude modulated due to the modulation parameters $\beta_{s i}=0.0338(i \in[1,4])$ with $\phi_{\beta s 1}=\pi / 2, \phi_{\beta s 2}=0$, 


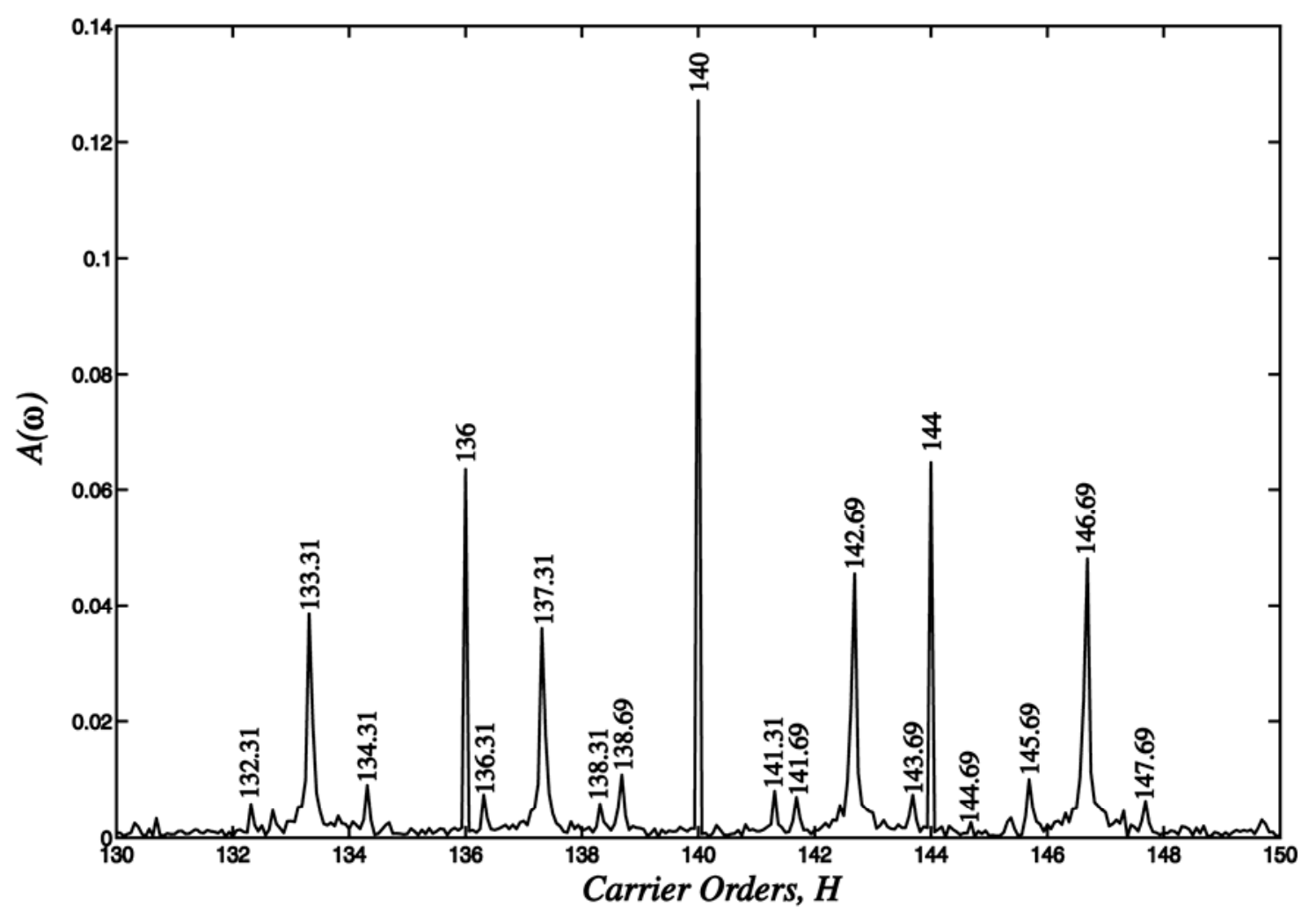

Figure 4.4: Predicted $A(\omega)$ spectra for the example planetary gear set with a rotating carrier under the influence of AM due to planet-1 run-out error with $\beta_{p 1}=0.034$, $\phi_{\beta p 1}=3 \pi / 2, \vartheta_{p 1}=0.033, \phi_{\vartheta p 1}=\pi / 2, \kappa_{p 1}=0.055, \phi_{\kappa p 1}=3 \pi / 2, \tau_{p 1}=0.049$ and $\phi_{\tau p 1}=\pi / 2$. 
$\phi_{\beta s 3}=3 \pi / 2, \quad \phi_{\beta s 4}=\pi \quad$ and $\quad \kappa_{s i}=0.0547 \quad(i \in[1,4]) \quad$ with $\quad \phi_{\kappa s 1}=\pi / 2, \quad \phi_{\kappa s 2}=0$, $\phi_{\kappa s 3}=3 \pi / 2, \phi_{\kappa s 4}=\pi$. Predicted $F_{s p 1}(\omega)$ and $F_{r p 1}(\omega)$ for this case are shown in Fig.

4.5. Sideband orders at $H=138.25$ and 141.75 are evident in these force spectra. They correspond to orders $H=H_{m} \pm H_{S / c}$ where $H_{s / c}=Z_{r} / Z_{S}=1.75$. The corresponding acceleration spectrum $A(\omega)$ is shown in Fig. 4.6. Here, besides the orders at $H=H_{m} \pm N=136$ and 144 those are due to rotation of the carrier, some additional sidebands appear at $H=H_{m} \pm H_{p / c} \pm n$ due to the amplitude modulation of the sidebands shown in Fig. 4.6. Orders at $H=139.25$ and 140.75 are of this kind. It is also noted here that, among these orders, $H=H_{m} \pm H_{S / c} \pm 1 \pm N$ (e.g. orders 135.25, 136.75, 143.25 and 144.75) have reasonably large amplitudes.

Likewise, a non-zero $E_{r}$ on the ring gear causes dynamic mesh forces $F_{s p 1}(\omega)$ and $F_{r p 1}(\omega)$ to modulate. Force modulation sidebands always appear at orders $H_{m} \pm H_{r / c}$ where $H_{r / c}=1$ as ring gear is fixed in this configuration. The corresponding $A(\omega)$ spectrum contains some additional sidebands at $H=H_{m} \pm H_{r / c} \pm n$. Figures for the case of ring gear error are not included here since they are qualitatively similar to those for the sun and planet gear errors. Instead, predictions for a case when $E_{p 1}=E_{s}=E_{r}=20 \mu \mathrm{m} \quad$ (all other $E_{p i}=0$ and $\left.\varepsilon_{p i}=\varepsilon_{s}=\varepsilon_{r}=0\right)$ are included here with all of the corresponding modulation parameters specified above used simultaneously. The resultant mesh force spectra shown in Fig. 4.7 indicate that the sidebands created due to each individual error are linearly superimposed. 

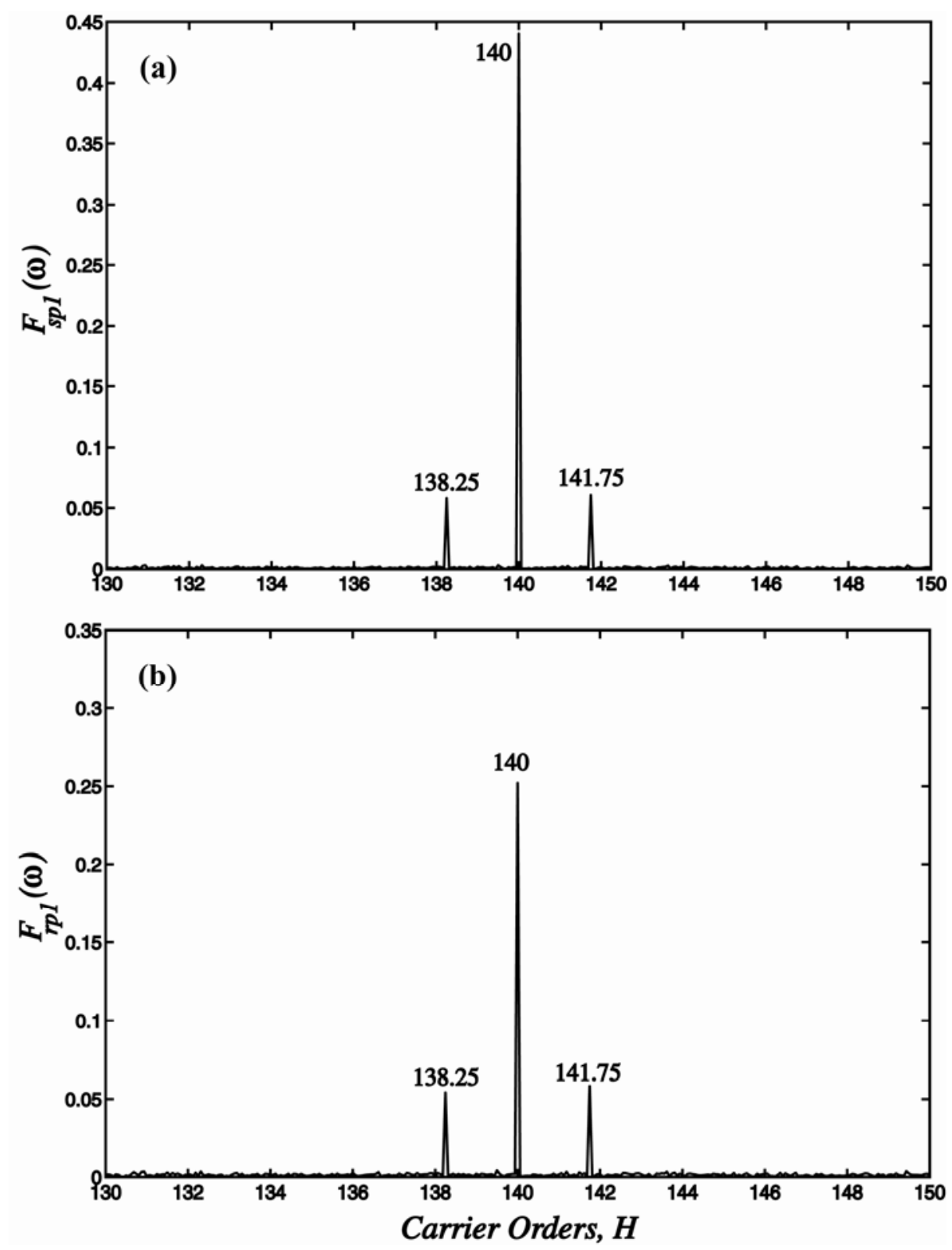

Figure 4.5: Predicted (a) $F_{s p 1}(\omega)$ and (b) $F_{r p 1}(\omega)$ spectra for the example planetary gear set under the influence of AM due to sun gear run-out error with $\beta_{s i}=0.0338(i \in[1,4])$, $\phi_{\beta s 1}=\pi / 2, \phi_{\beta s 2}=0, \phi_{\beta s 3}=3 \pi / 2, \phi_{\beta s 4}=\pi$ and $\kappa_{s i}=0.0547(i \in[1,4]), \phi_{\kappa s 1}=\pi / 2$, $\phi_{\mathrm{KS} 2}=0, \phi_{\mathrm{KS} 3}=3 \pi / 2$ and $\phi_{\mathrm{KS} 4}=\pi$. 


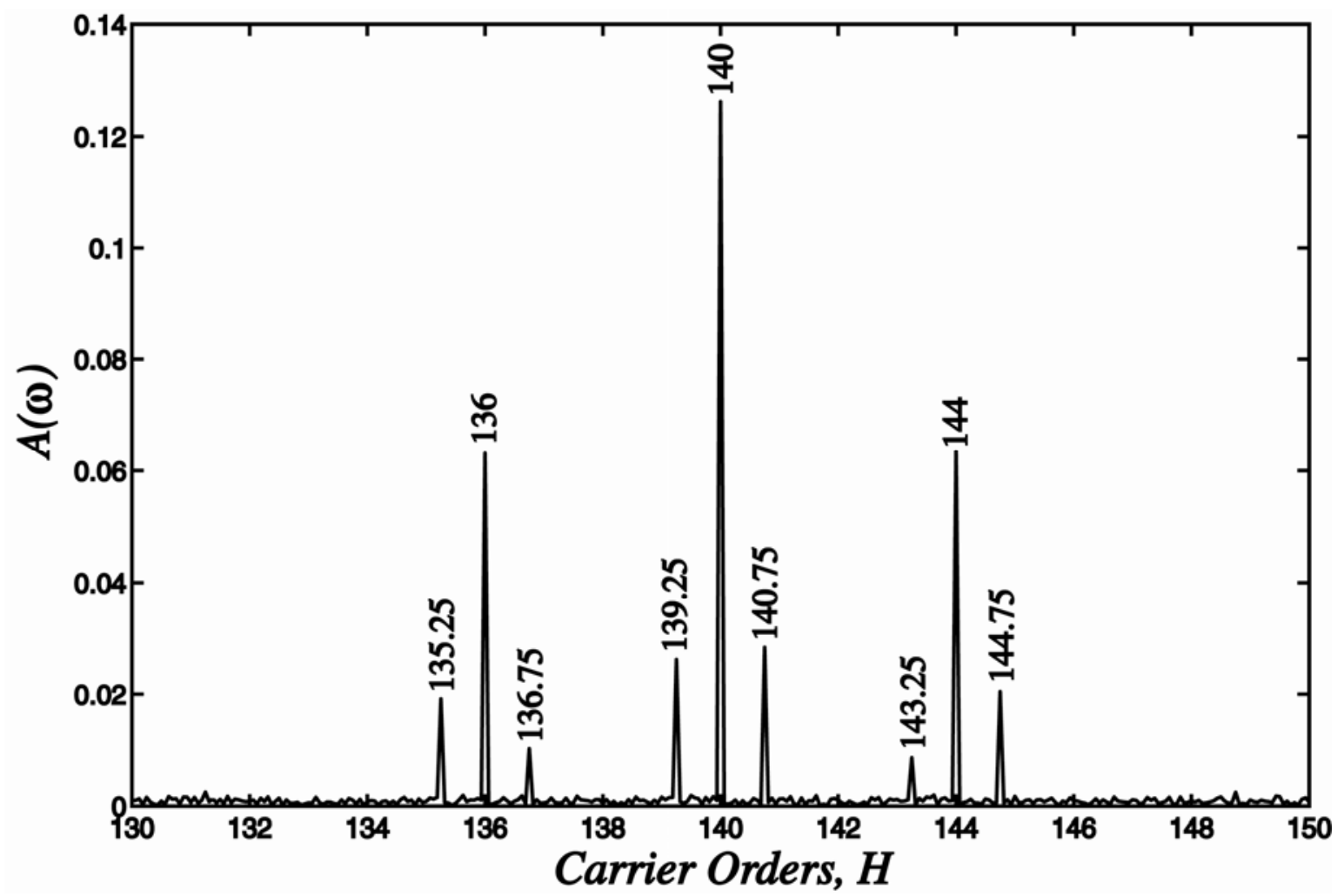

Figure 4.6: Predicted $A(\omega)$ spectra for the example planetary gear set with a rotating carrier under the influence of $\mathrm{AM}$ due to sun gear run-out error with $\beta_{S i}=0.0338$ $(i \in[1,4]), \phi_{\beta s 1}=\pi / 2, \phi_{\beta s 2}=0, \phi_{\beta s 3}=3 \pi / 2, \phi_{\beta s 4}=\pi$ and $\kappa_{s i}=0.0547 \quad(i \in[1,4])$, $\phi_{\mathrm{KS} 1}=\pi / 2, \phi_{\mathrm{KS} 2}=0, \phi_{\mathrm{KS} 3}=3 \pi / 2$ and $\phi_{\mathrm{KS} 4}=\pi$. 

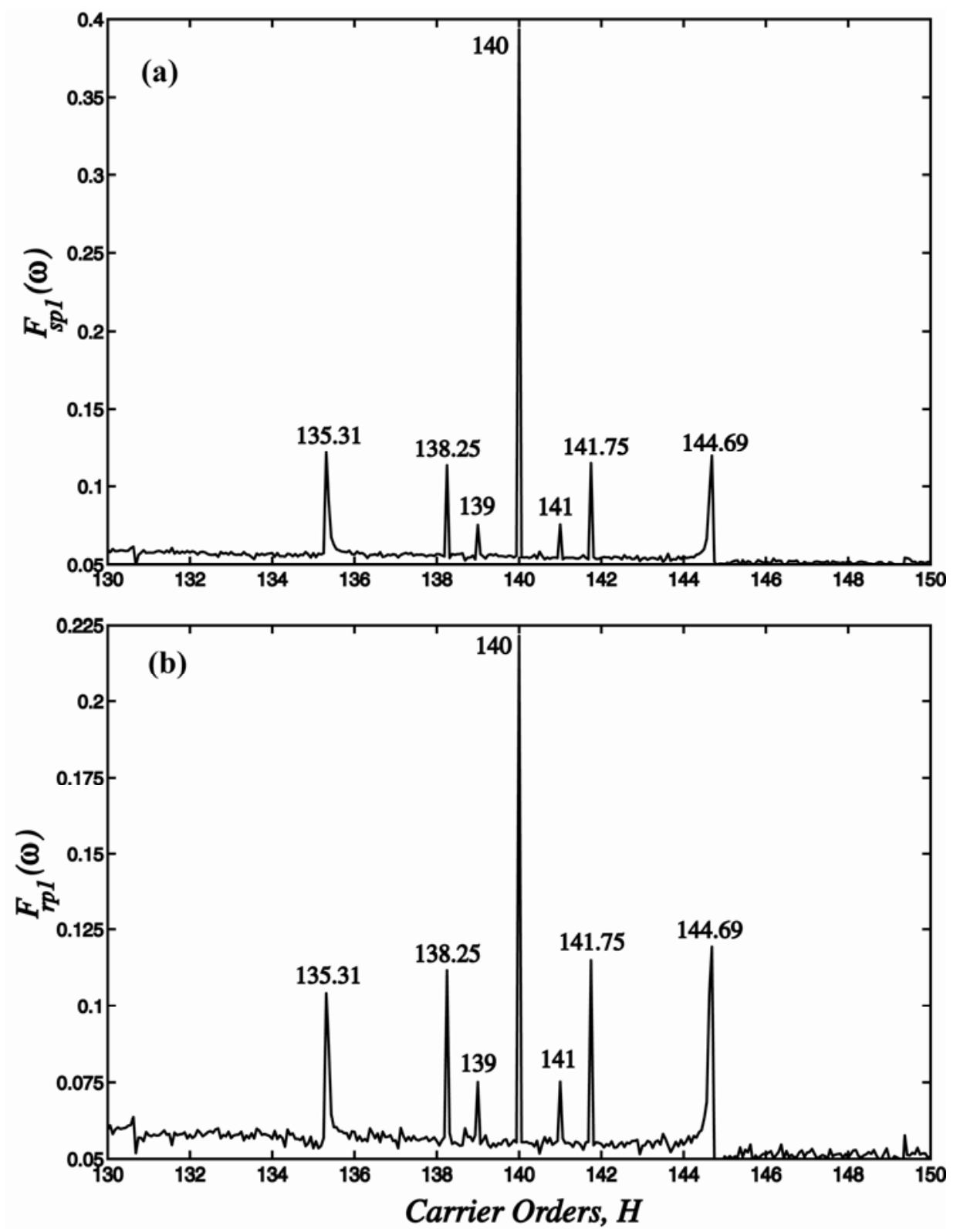

Figure 4.7: Predicted (a) $F_{s p 1}(\omega)$ and (b) $F_{r p 1}(\omega)$ spectra for the example planetary gear set under the influence of AM due to planet-1, sun and ring gear run-out errors. $\hat{\beta}_{r}=\hat{\kappa}_{r}=2.0(10)^{-5}(i \in[1,4]), \phi_{\hat{\beta} r 1}=\phi_{\hat{\tau} r 1}=\frac{3 \pi}{2}, \phi_{\hat{\beta} r 2}=\phi_{\hat{\kappa} r 2}=0, \phi_{\hat{\beta} r 3}=\phi_{\hat{\kappa} r 3}=\frac{\pi}{2}$, and $\phi_{\hat{\beta} r 4}=\phi_{\hat{\kappa} r 4}=\pi$, and sun and planet related parameters are as in Fig. 4.4 and 4.6. 


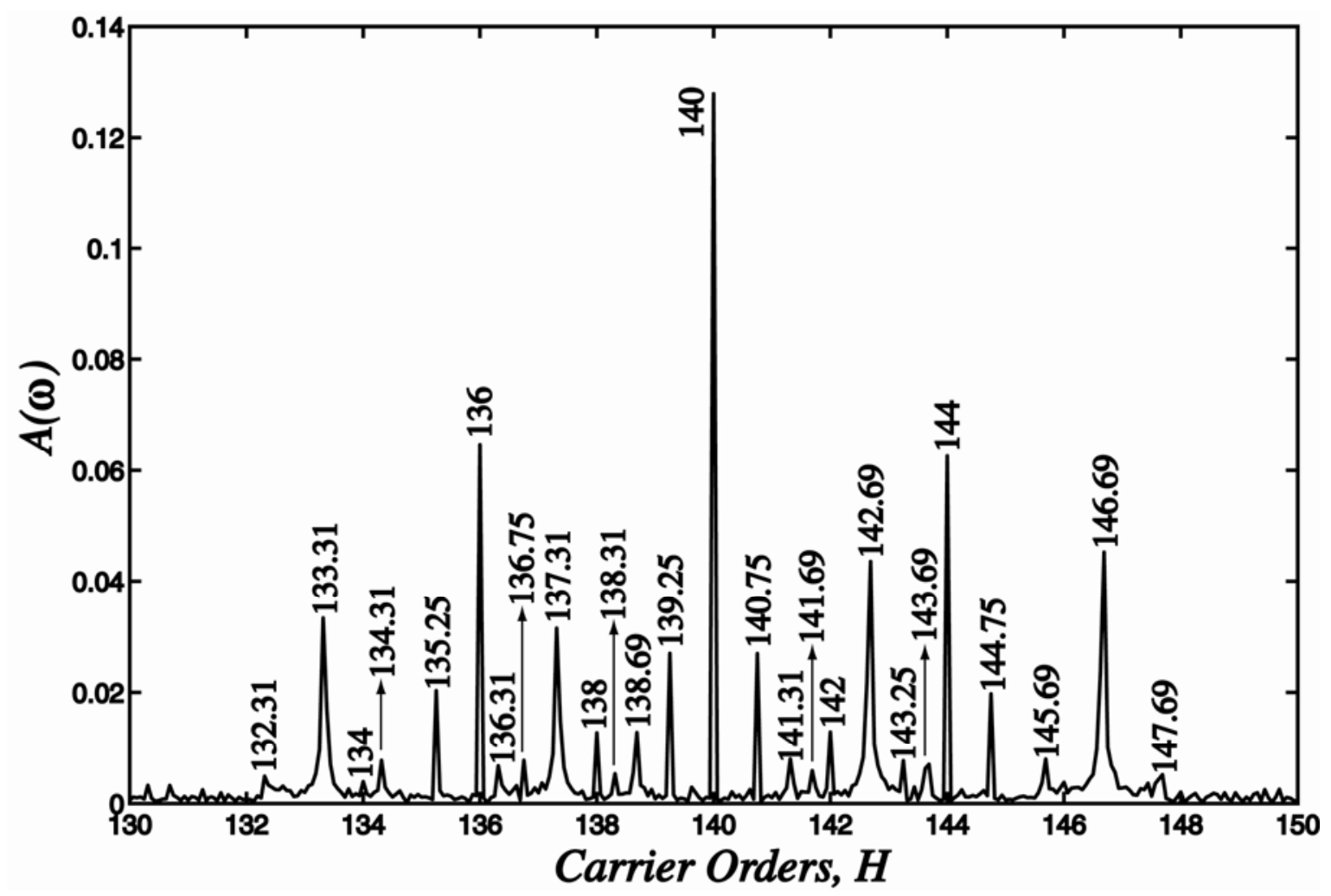

Figure 4.8: Predicted $A(\omega)$ spectra for the example planetary gear set with a rotating carrier under the influence of AM due to planet-1, sun and ring gear run-out errors. $\hat{\beta}_{r}=\hat{\kappa}_{r}=2.0(10)^{-5}(i \in[1,4]), \phi_{\hat{\beta} r 1}=\phi_{\hat{\tau} r 1}=\frac{3 \pi}{2}, \phi_{\hat{\beta} r 2}=\phi_{\hat{\kappa} r 2}=0, \phi_{\hat{\beta} r 3}=\phi_{\hat{\kappa} r 3}=\frac{\pi}{2}$, and $\phi_{\hat{\beta} r 4}=\phi_{\hat{\kappa} r 4}=\pi$, and sun and planet related parameters are as in Fig. 4.4 and 4.6. 
The same statement is true for the corresponding $A(\omega)$ as well, as shown in Fig. 4.8. Here, all of the sideband groups identified above for each error, $H_{m} \pm H_{p / c} \pm n$, $H_{m} \pm H_{r / c} \pm n$ and $H_{m} \pm H_{p / c} \pm n$ ( $n$ : integer) coexist. It is also noted here that this $A(\omega)$ spectrum can be used to identify any sources of error in a planetary gear set as long as orders $H_{p / c}, H_{s / c}$ and $H_{r / c}$ are distinct. Only time these orders are not distinct is when $Z_{p}=Z_{s}$, resulting in $H_{p / c}=H_{s / c}$. In this special case, it would not be possible to separate the sideband orders caused by sun gear and planet errors.

\section{Frequency Modulations}

Up to this point in this example analysis, only the amplitude modulations of the excitation functions due to manufacturing errors were considered. The same procedure will be used next to investigate the impact of the FM parameters in Eq. (4.14), (4.15), (4.17) and (4.18), with AM parameters intentionally turned off. For the first case of the planet gear run-out error of $E_{p 1}=20 \mu \mathrm{m}$ with $\varepsilon_{p 1}=0$, FM parameters are estimated as

$\hat{\beta}_{p 1}=\hat{\vartheta}_{p 1}=\hat{\kappa}_{p 1}=\hat{\tau}_{p 1}=8.5(10)^{-5}, \quad \phi_{\hat{\beta} p 1}=3 \pi / 2, \quad \phi_{\hat{\vartheta} p 1}=\pi / 2, \quad \phi_{\hat{\kappa} p 1}=3 \pi / 2, \quad$ and $\phi_{\hat{\tau} p 1}=\pi / 2$. The resultant $F_{s p 1}(\omega)$ and $F_{r p 1}(\omega)$ are shown in Fig. 4.9. Instead of creating a single pair of sidebands as it was in the case of AM in Fig. 4.3, a large number of sidebands are created by the frequency modulation of the gear mesh excitations. The FM sidebands ultimately appear at orders $H_{m} \pm n H_{p / c}$. However, in cases where relatively large frequency modulation parameters are used, additional orders show up 
possibly at orders $H_{m} \pm n H_{p / c} \pm \hat{\beta}_{p i} H_{m}$. It is also observed from these two spectra that sidebands with significant amplitudes range from $H=115$ to 165 . The corresponding $A(\omega)$ spectrum at a fixed transducer location is shown in Fig. 4.10. Here, fundamental mesh harmonic order at $H_{m}=140$ is split into orders 138 and 142. In Fig. 4.10(b), which is nothing but the wideband version of the spectrum given in Figure 4.10(a), significant sidebands at orders $H_{m} \pm n H_{p / c} \pm n N$ appear additional to the ones appearing at orders $H_{m} \pm n H_{p / c}$.

Secondly, a sun gear run-out error with $E_{S}=20 \mu m$ starting at the initial position angle of $\varepsilon_{S}=0^{\circ}$ is introduced to the system. Only the FM parameters $\left(\hat{\beta}_{s}=\hat{\kappa}_{s}=3.0(10)^{-5}, i \in[1,4], \phi_{\hat{\beta} s 1}=\phi_{\hat{\kappa} s 1}=\frac{\pi}{2}, \phi_{\hat{\beta} s 2}=\phi_{\hat{\kappa} s 2}=0, \phi_{\hat{\beta} s 3}=\phi_{\hat{\kappa} s 3}=\frac{3 \pi}{2}\right.$ and $\left.\phi_{\hat{\beta} S 4}=\phi_{\hat{K} S 4}=\pi\right)$ are included here in order to demonstrate the sole influence of frequency modulations. The predicted $F_{s p 1}(\omega)$ and $F_{r p 1}(\omega)$ are shown in Fig. 4.11. The FM sidebands typically appear at orders $H_{m} \pm n H_{s / c}$. Meanwhile, Fig. 4.12 shows the corresponding $A(\omega)$ spectrum, exhibiting sidebands in a narrower order range of $H_{m} \pm 10$, which represents a much narrower frequency range than that of Fig. 4.10. This is simply because the value of the modulation parameter $\hat{\beta}_{S}$ in Fig. 4.11 and 4.12 is smaller than the value of $\hat{\beta}_{p i}$ corresponding to Fig. 4.9 and 4.10. Fig. 4.12(a) exhibits dominant sidebands, besides the most expected peaks at $H_{m} \pm N$, appearing at orders $H_{m} \pm n H_{s / c} \pm 2$ as well as $H_{m} \pm n H_{s / c} \pm 2 \pm n$ and $H_{m} \pm n H_{s / c} \pm 2 \pm N$. 

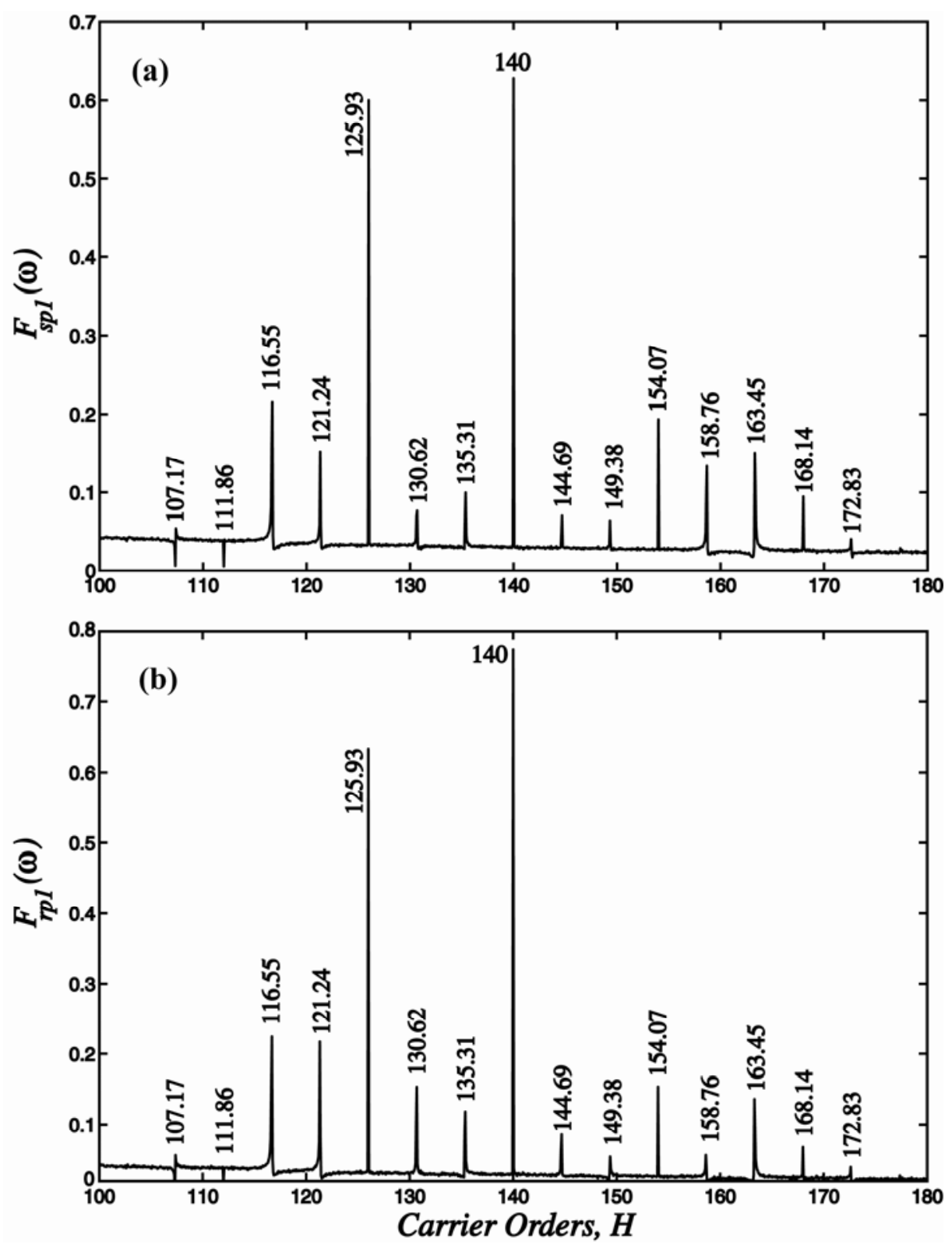

Figure 4.9: Predicted (a) $F_{s p 1}(\omega)$ and (b) $F_{r p 1}(\omega)$ spectra for the example planetary gear set when only FM are present due to planet-1 run-out with $\hat{\beta}_{p 1}=\hat{\vartheta}_{p 1}=\hat{\kappa}_{p 1}=\hat{\tau}_{p 1}=8.5(10)^{-5}, \quad \phi_{\hat{\beta} p 1}=3 \pi / 2, \quad \phi_{\hat{\vartheta} p 1}=\pi / 2, \quad \phi_{\hat{\kappa} p 1}=3 \pi / 2 \quad$ and $\phi_{\hat{\tau} p 1}=\pi / 2$. 


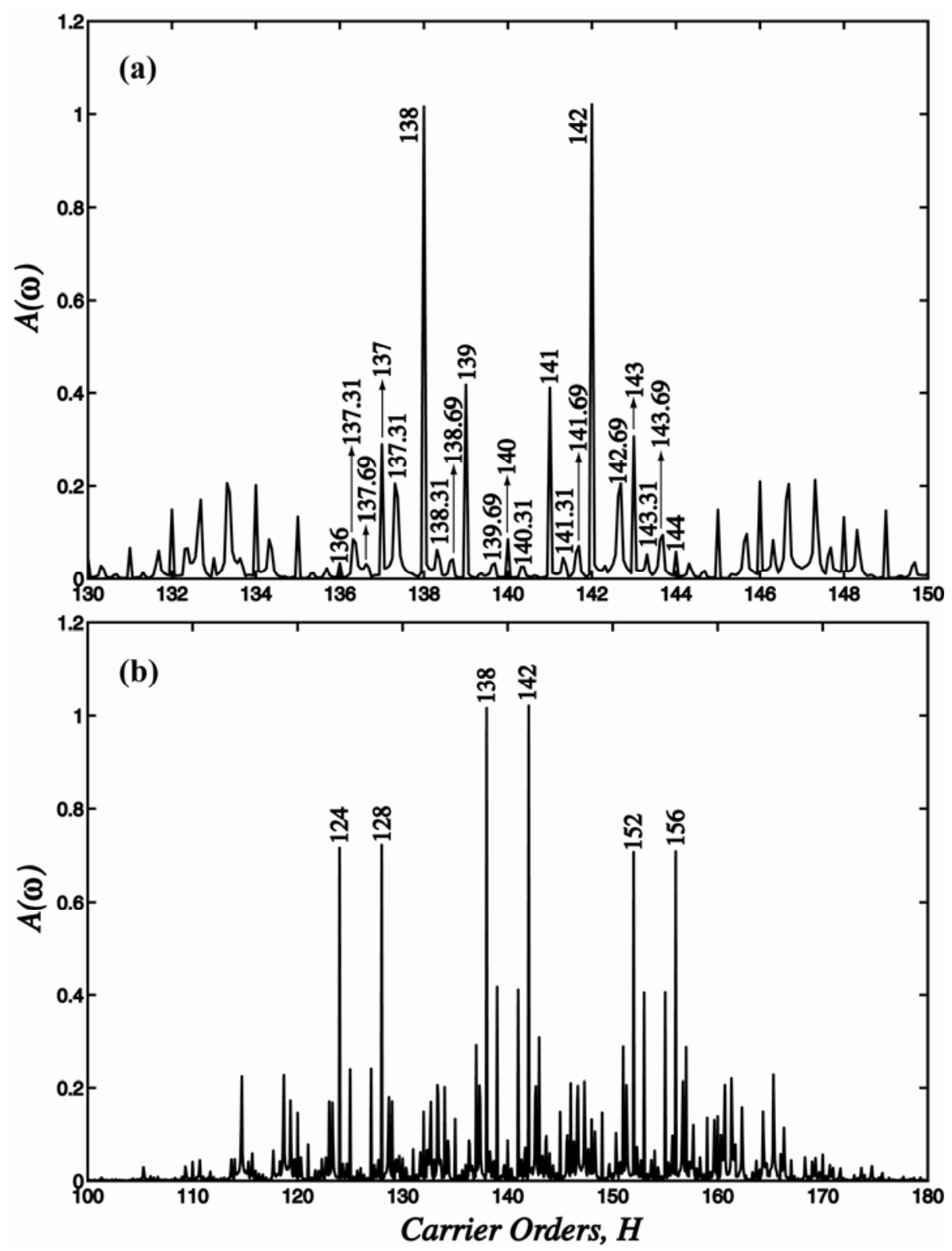

Figure 4.10: Predicted $A(\omega)$ spectrum for the example planetary gear set when only FM are present due to planet-1 run-out with $\hat{\beta}_{p 1}=\hat{\vartheta}_{p 1}=\hat{\kappa}_{p 1}=\hat{\tau}_{p 1}=8.5(10)^{-5}, \phi_{\hat{\beta} p 1}=3 \pi / 2$, $\phi_{\hat{\vartheta} p 1}=\pi / 2, \phi_{\hat{\kappa} p 1}=3 \pi / 2$ and $\phi_{\hat{\tau} p 1}=\pi / 2$. (a) A zoomed in view in the vicinity of the fundamental gear mesh frequency and (b) the same spectrum with a wider frequency range. 

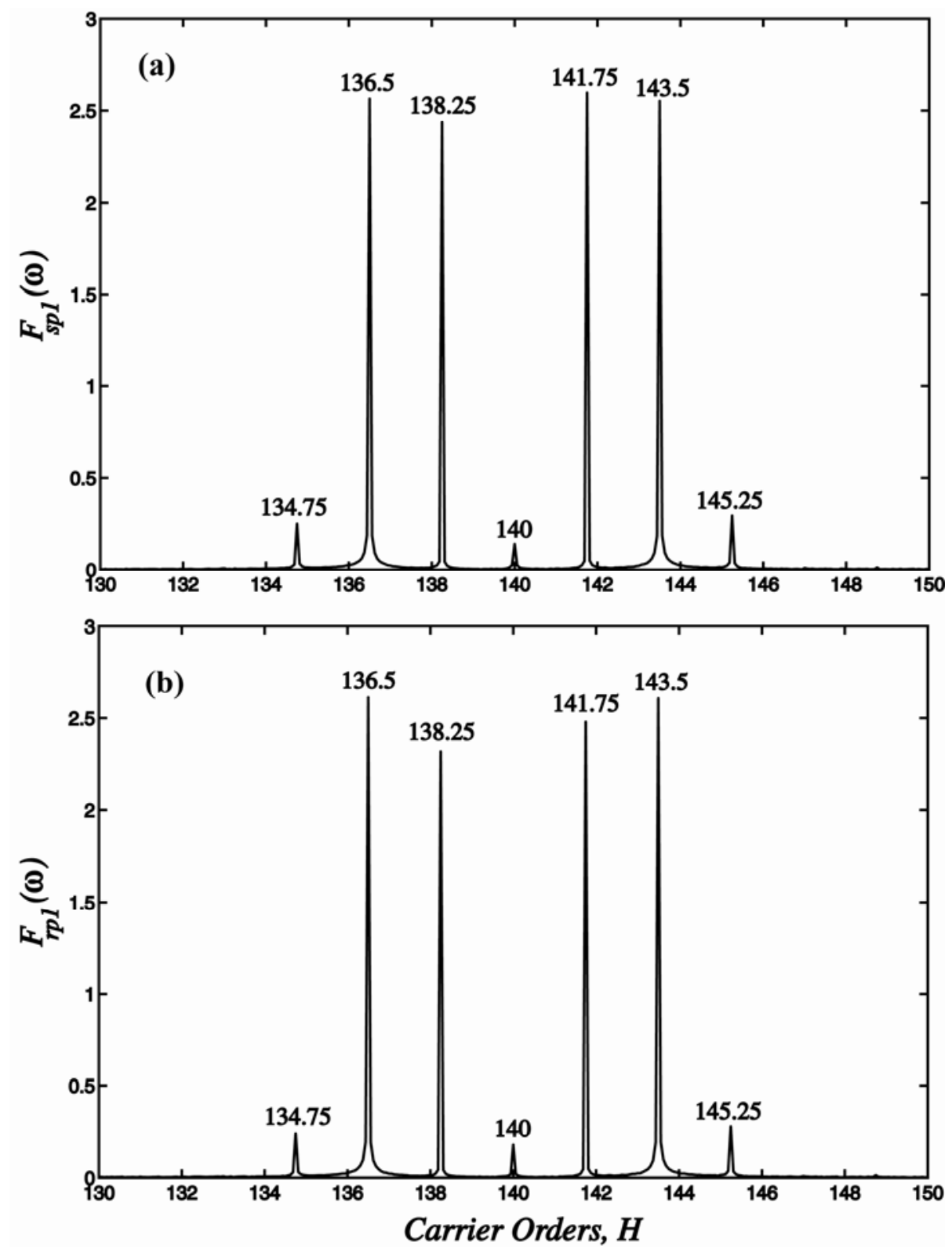

Figure 4.11: Predicted (a) $F_{s p 1}(\omega)$ and (b) $F_{r p 1}(\omega)$ spectra for the example planetary gear set when only FM are present due to sun gear run-out with $\hat{\beta}_{s}=\hat{\kappa}_{S}=3.0(10)^{-5}$, $i \in[1,4], \phi_{\hat{\beta} s 1}=\phi_{\hat{\kappa} s 1}=\frac{\pi}{2}, \phi_{\hat{\beta} s 2}=\phi_{\hat{\kappa} s 2}=0, \phi_{\hat{\beta} s 3}=\phi_{\hat{\kappa} s 3}=\frac{3 \pi}{2}$ and $\phi_{\hat{\beta} s 4}=\phi_{\hat{\kappa} s 4}=\pi$. 

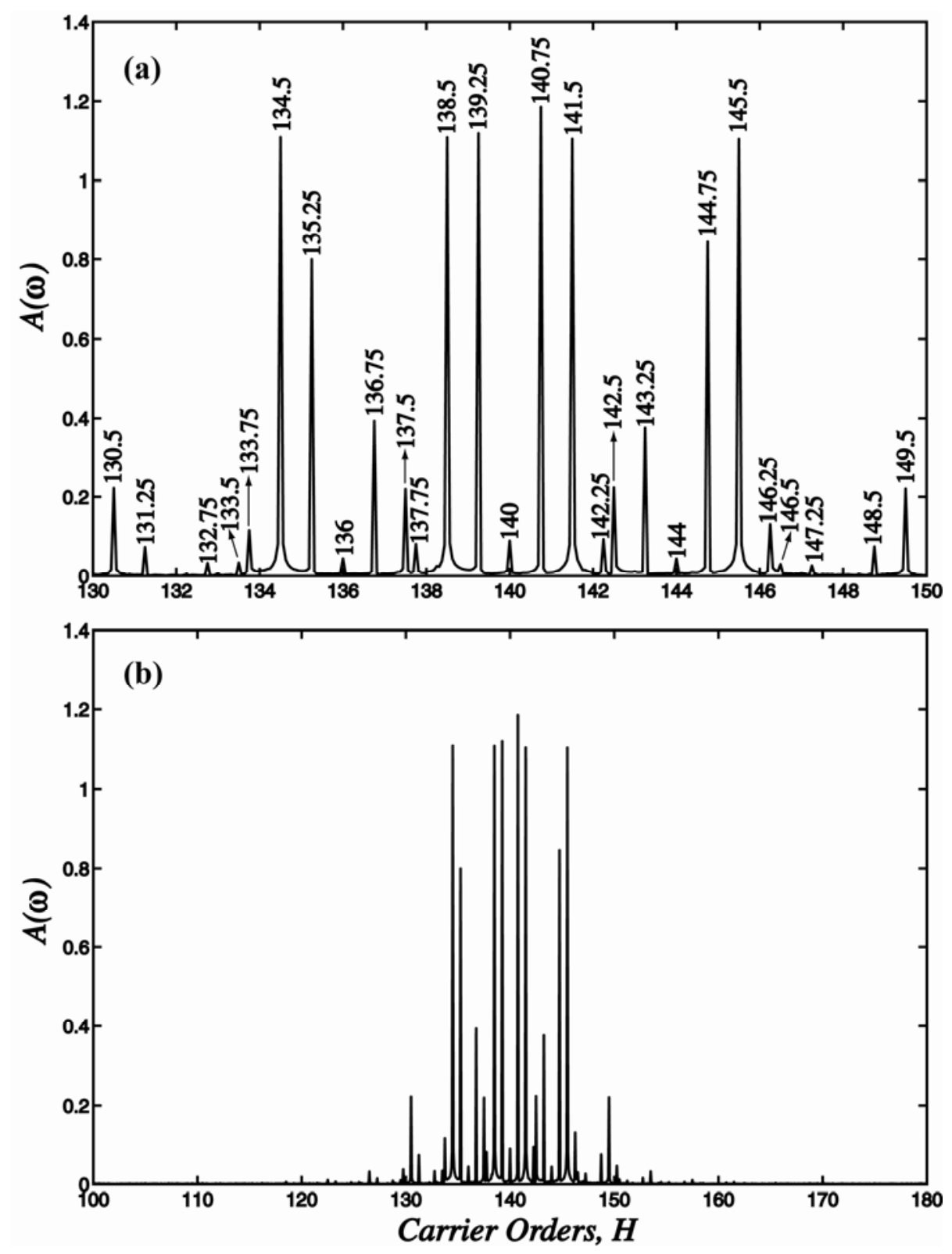

Figure 4.12: Predicted $A(\omega)$ spectrum for the example planetary gear set when only FM are present due to sun gear run-out with parameters specified in Fig. 4.11. (a) A zoomed in view in the vicinity of the fundamental gear mesh frequency and (b) the same spectrum with a wider frequency range. 
Similar to the previous two cases, a non-zero $E_{r}$ on the ring gear causes gear mesh force sideband orders at $H_{m} \pm n H_{r / c}$. The corresponding $A(\omega)$ spectrum contains some additional sidebands at $H=H_{m} \pm 2 n H_{r / c} \pm n$ for the specific case of $N=$ even, equally-spaced and in phase planets. Again, figures for the case of ring gear error are not included here since they are qualitatively similar to those for the sun and planet gear errors. Instead, FM predictions for a case when $E_{p 1}=E_{s}=E_{r}=20 \mu \mathrm{m}$ (all other $E_{p i}=0$ and $\left.\varepsilon_{p i}=\varepsilon_{s}=\varepsilon_{r}=0\right)$ are included here with all of the corresponding frequency modulation parameters specified above in addition to $\hat{\beta}_{r}=\hat{\kappa}_{r}=2.0(10)^{-5}(i \in[1,4])$, $\phi_{\hat{\beta} r 1}=\phi_{\hat{\tau} r 1}=\frac{3 \pi}{2}, \phi_{\hat{\beta} r 2}=\phi_{\hat{\kappa} r 2}=0, \phi_{\hat{\beta} r 3}=\phi_{\hat{\kappa} r 3}=\frac{\pi}{2}$, and $\phi_{\hat{\beta} r 4}=\phi_{\hat{\kappa} r 4}=\pi$. The dynamic gear mesh force spectra corresponding to this case are shown in Fig. 4.13, which are a linear superposition of the spectra for individual errors as nonlinear behavior such as tooth separations are not in effect. The same can be said for $A(\omega)$ shown in Fig. 4.14 as well. Here, all of the FM sideband groups identified above for each individual error, $H_{m} \pm H_{p / c} \pm n, H_{m} \pm H_{r / c} \pm n$ and $H_{m} \pm H_{s / c} \pm n$, coexist such that each sideband can be related to a particular error since the values of $H_{p / c}, H_{s / c}$ and $H_{r / c}$ are distinct. It is also noted here that sideband activity due to FM is richer and more complex than that of AM sources. It can also be stated that the FM sidebands are very sensitive to parameters $\hat{\beta}_{j}$ and $\hat{\kappa}_{j}(j=s, r, p 1, p 2, \ldots, p N)$, emphasizing the importance of determining their values accurately from the actual gear errors $E_{j}$ using quasi-static deformable-body gear contact models. 

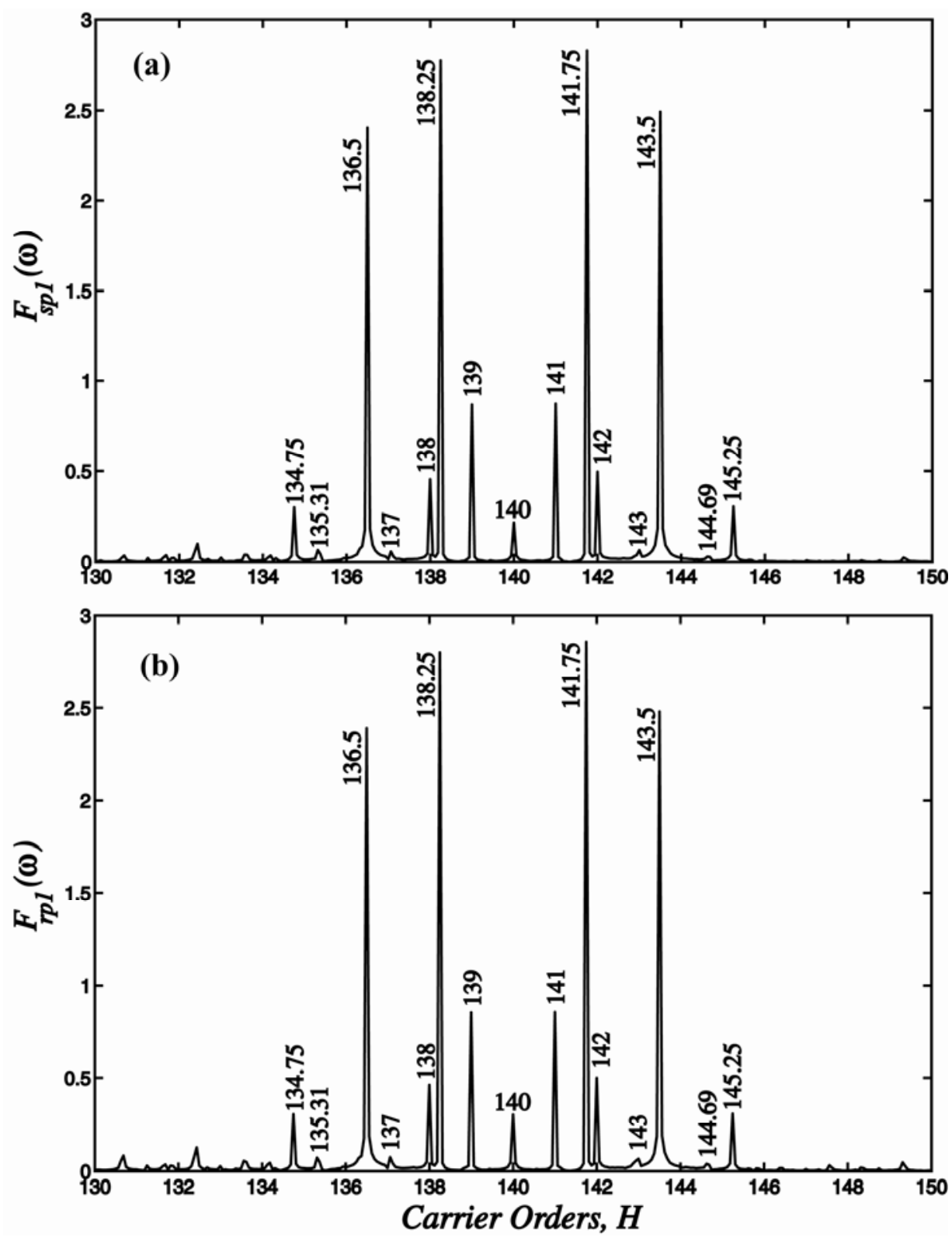

Figure 4.13: Predicted (a) $F_{s p 1}(\omega)$ and (b) $F_{r p 1}(\omega)$ spectra for the example planetary gear set when only FM are present due to planet-1, sun gear and ring gear run-out errors. $\hat{\beta}_{r}=\hat{\kappa}_{r}=2.0(10)^{-5}(i \in[1,4]), \phi_{\hat{\beta} r 1}=\phi_{\hat{\tau} r 1}=\frac{3 \pi}{2}, \phi_{\hat{\beta} r 2}=\phi_{\hat{\kappa} r 2}=0, \phi_{\hat{\beta} r 3}=\phi_{\hat{\kappa} r 3}=\frac{\pi}{2}$, and $\phi_{\hat{\beta} r 4}=\phi_{\hat{\kappa} r 4}=\pi$ and sun and planet related parameters are as in Fig. 4.10 and 4.12. 

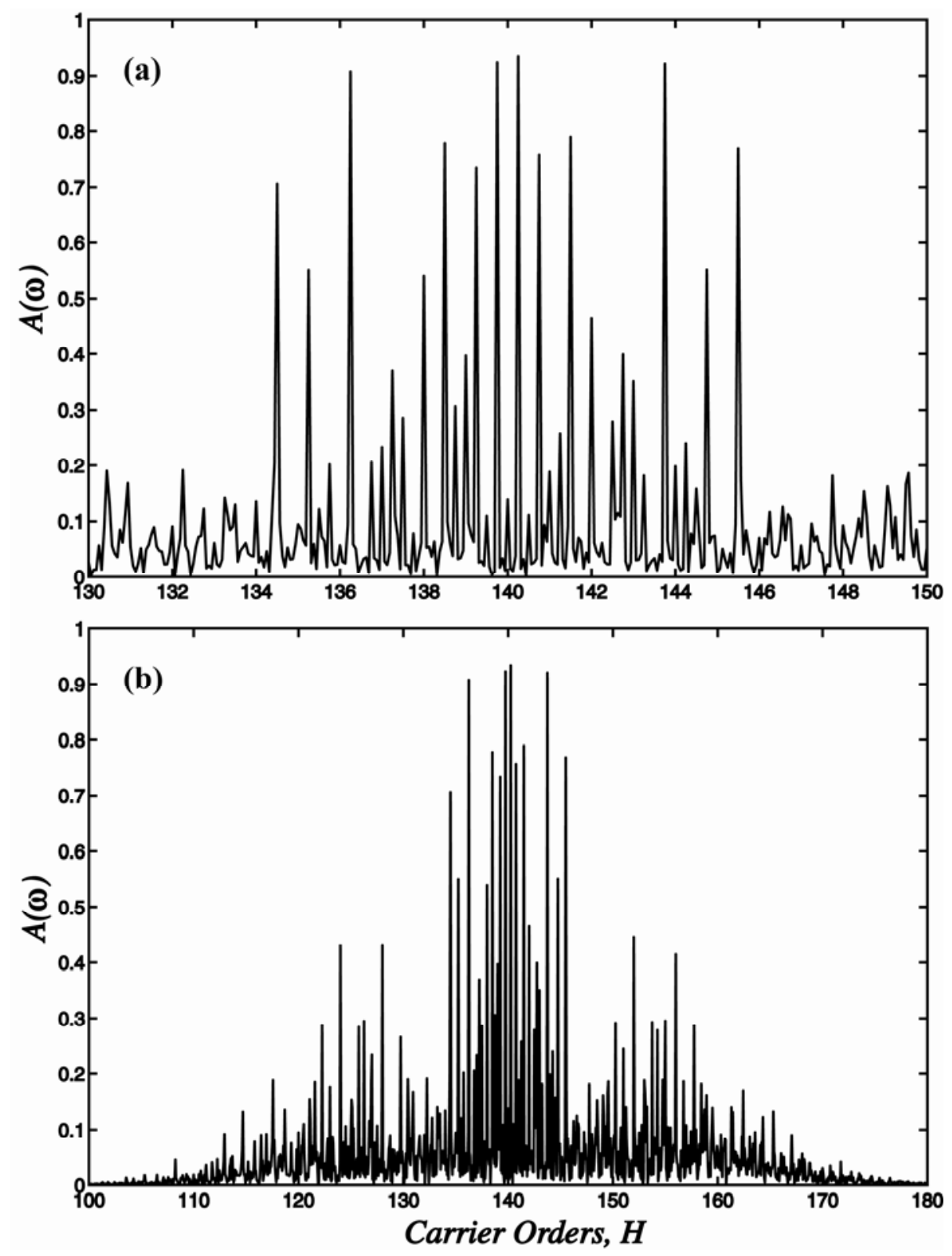

Figure 4.14: Predicted $A(\omega)$ spectrum for the example planetary gear set when only FM when only FM are present due to planet-1, sun gear and ring gear run-out errors with parameters specified in Fig. 4.13. (a) A zoomed in view in the vicinity of the fundamental gear mesh frequency and (b) the same spectrum with a wider frequency range. 


\subsubsection{Planetary Gear Set Modulations Near a Resonance}

Most of the vibration spectra measured from planetary transmissions exhibit asymmetric sidebands around the gear mesh orders. In Chapter 3 , the rotation of the carrier was shown to be one of the sources of such asymmetric sidebands, unless the gear set has equally-spaced and in-phase planets as the example planetary gear set considered in this study. In agreement with this, the $A(\omega)$ spectra presented in the previous section exhibited mostly symmetric sidebands. In this section, the sidebands of this example system will be predicted at a near-resonance region to demonstrate that dynamic effects might be attributed to some of the asymmetric sidebands as well.

Using the same AM parameters as those used in Fig. 4.3 and 4.4 to correspond to the eccentricity of planet 1 only, the dynamic response is predicted here at a gear mesh frequency of $950 \mathrm{~Hz}$ that is near the natural frequency at $982 \mathrm{~Hz}$. Figure 4.15 shows the predicted $F_{s p 1}(\omega)$ and $F_{r p 1}(\omega)$. Here, the spectra that exhibit asymmetry about the gear mesh order $H_{m}=140$, contrasting the symmetric spectra presented in Fig. 4.3 for the same system at an off-resonance condition. The right side sideband $H_{m}+H_{p / c}=144.69$ has a much larger amplitude than components at $H_{m}$ and $H_{m}-H_{p / c}$ since this order coincides with the natural frequency at $982 \mathrm{~Hz}$. Similarly, the $A(\omega)$ spectrum shown in Fig. 4.16 is also asymmetric about $H_{m}$ for the same reason. This clearly indicates that dynamic conditions might be one of the reasons for asymmetry as well. 

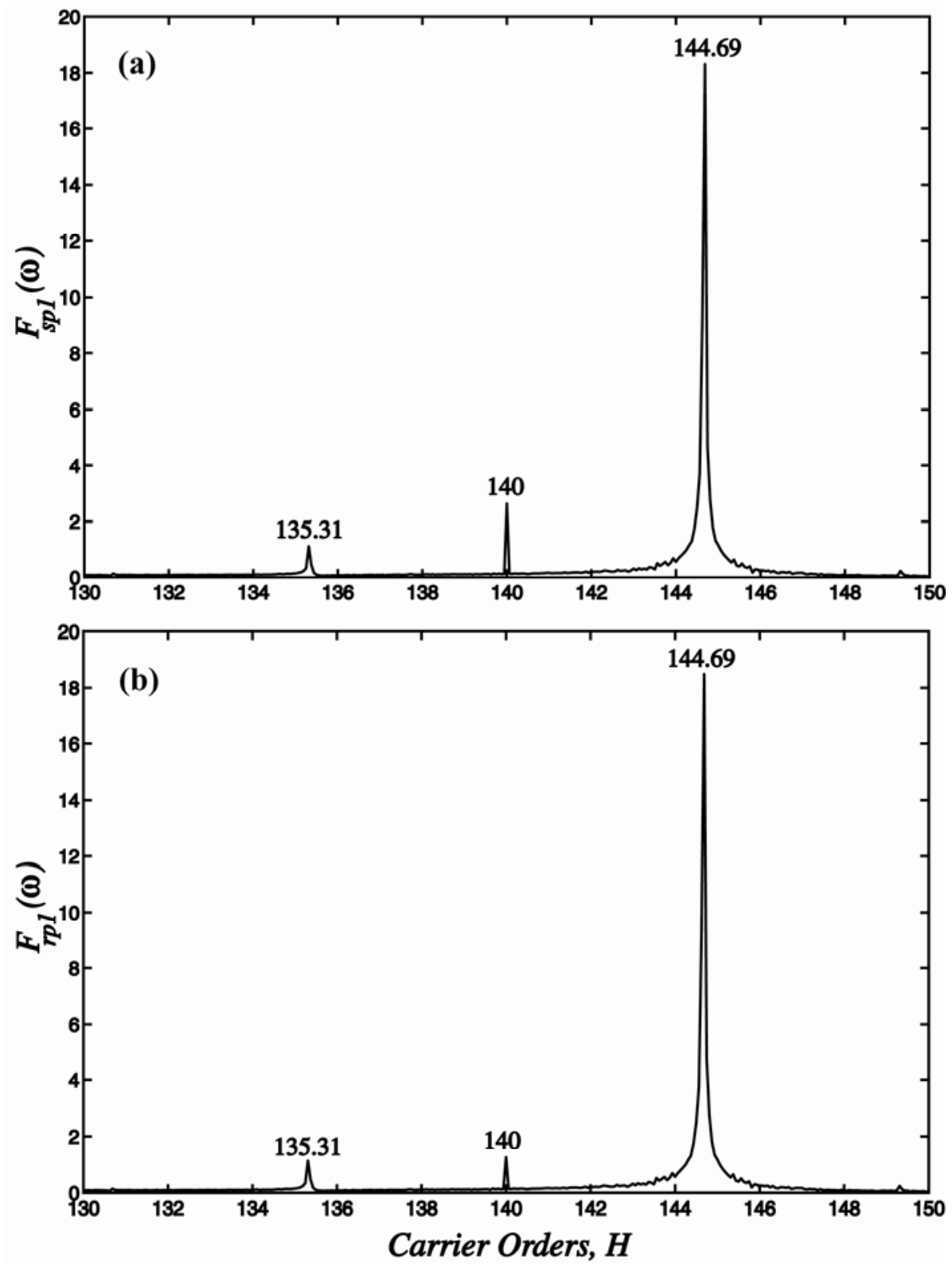

Figure 4.15: Predicted (a) $F_{s p 1}(\omega)$ and (b) $F_{r p 1}(\omega)$ spectra for the example planetary gear set under the influence of AM due to planet-1 run-out error with $\beta_{p 1}=0.034$, $\phi_{\beta p 1}=3 \pi / 2, \quad \vartheta_{p 1}=0.033, \quad \phi_{\vartheta p 1}=\pi / 2, \quad \kappa_{p 1}=0.055, \quad \phi_{\kappa p 1}=3 \pi / 2, \quad \tau_{p 1}=0.049$, $\phi_{\tau p 1}=\pi / 2$ and $\omega_{m}=151.2 \mathrm{rad} / \mathrm{s}$. 


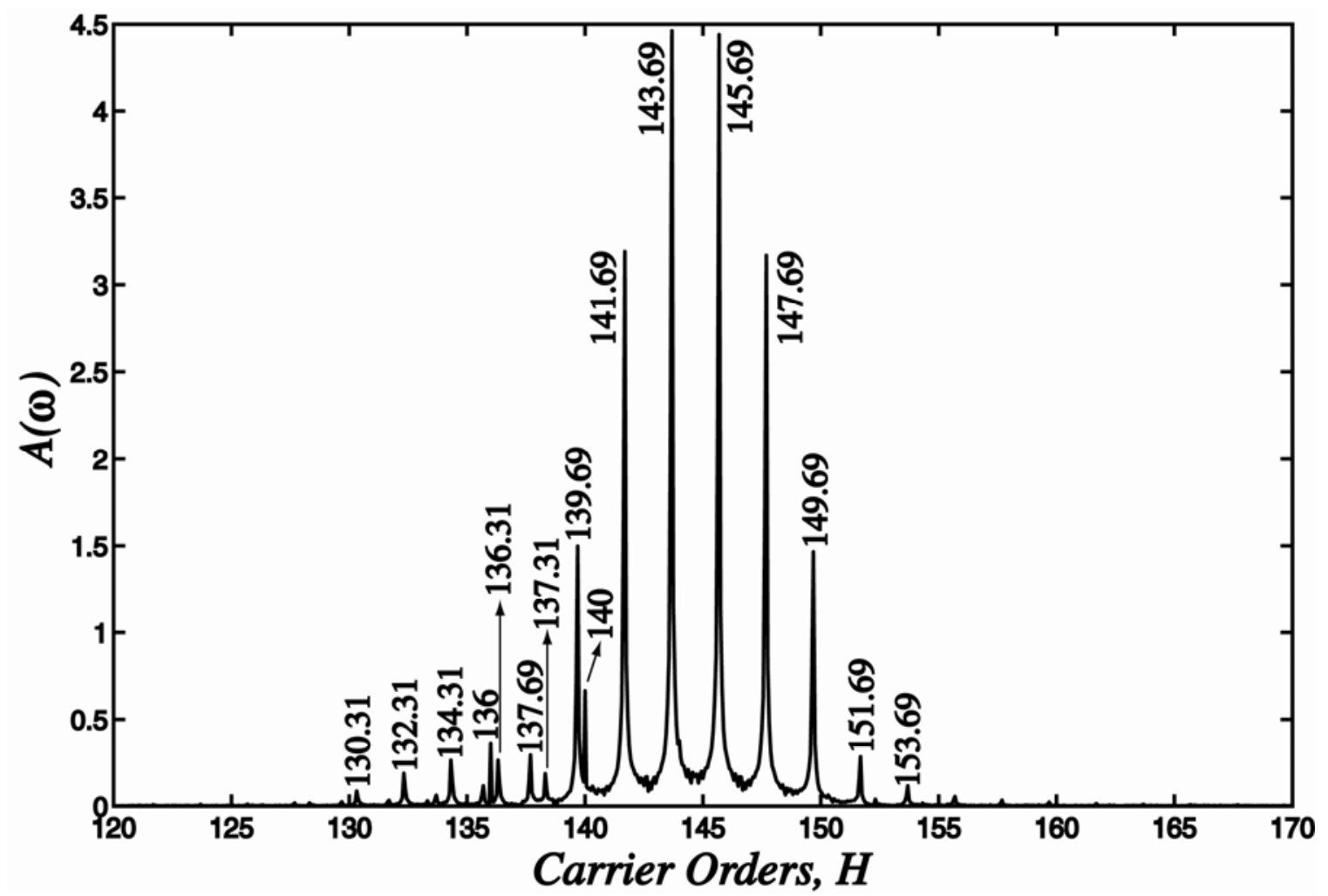

Figure 4.16: Predicted $A(\omega)$ spectrum for the example planetary gear set under the influence of AM due to planet-1 run-out error with parameters specified in Fig. 4.15. 


\subsection{Experimental Validation}

In this section, the proposed dynamic model will be used to describe the sidebands observed in experimental spectra of Chapter 2. In Chapter 3, the primary aim was to validate the predictions of the proposed simplified AM model due to carrier rotation. That model was able to describe only a subset of the measured sidebands, forming the motivation for this study. An example measured acceleration spectrum from this earlier study will be compared to the predictions of the proposed model to demonstrate the ability of the model in accounting for the gear manufacturing errors.

One of the three example experimental gear sets from Chapter 2 with four unequally spaced planets ( $N=4$ with $\psi_{i}=0,90.909^{\circ}, 180^{\circ}$ and $270.909^{\circ}$ ) will be considered to here. For this gear set with $Z_{r}=125, Z_{s}=73$ and $Z_{p}=26$, an arbitrary planet phasing existed $\left(Z_{r} \psi_{i}=0,1.1325 \pi, \pi\right.$ and $\left.0.1312 \pi\right)$ such that it fall into the Case (v) category in Chapter 3 of this dissertation. Figure 4.17 shows an example measured acceleration spectrum collected when this gear set was operating at $T_{S}=400$ $\mathrm{Nm}$ and $\Omega_{\mathrm{s}}=500 \mathrm{rpm}$. The sidebands of this spectrum at integer orders can be attributed to the amplitude modulations due to the carrier rotation. However, the sidebands appearing at orders such as $116.19,118.19,119.19$ and 126.81 can be thought of as a direct result of certain gear manufacturing errors. These orders are associated with the planet run-out errors.

The proposed model is used to simulate this gear set at the same operating condition with the AM and FM parameters associated with the run-out error of a planet 
gear $\left(E_{p 1}=20 \mu \mathrm{m}, \varepsilon_{p 1}=0\right)$. Firstly, the AM parameters corresponding to this planet gear run-out error are predicted as $\beta_{p 1}=0.0342, \quad \phi_{\beta p 1}=3 \pi / 2, \quad \vartheta_{p 1}=0.0335$, $\phi_{\vartheta p 1}=\pi / 2, \quad \kappa_{p 1}=0.0552, \quad \phi_{\kappa p 1}=3 \pi / 2, \quad \tau_{p 1}=0.0496 \quad$ and $\quad \phi_{\tau p 1}=\pi / 2 \quad[4.21]$. Predicted $A(\omega)$ spectrum for AM only analysis is shown in Fig. 4.18 and generates the measured sidebands at orders such as 116.19, 118.19, 119.19 and 126.81 (each at $\left.H_{m} \pm H_{p / c} \pm n\right)$ in addition to even integer orders such as 120,122 and 124 caused by the carrier rotation. Secondly, the same analysis is repeated with the corresponding FM parameters $\quad$ only $\quad\left(\hat{\beta}_{p 1}=\hat{\vartheta}_{p 1}=\hat{\kappa}_{p 1}=\hat{\tau}_{p 1}=1.0(10)^{-4}, \quad \phi_{\hat{\beta} p 1}=\phi_{\hat{\kappa} p 1}=\frac{3 \pi}{2} \quad\right.$ and $\left.\phi_{\hat{\vartheta} p 1}=\phi_{\hat{\tau} p 1}=\frac{\pi}{2}\right)$ to produce the spectrum shown in Fig. 4.19. In this spectrum, large sideband amplitudes are evident at odd integer orders, especially at orders 123 and 127. Finally, the predicted acceleration spectrum presented in Fig. 4.20 is due to both AM and FM effects of the planet run-out error. Comparison of Fig. 4.20 to Fig. 4.17 indicates clearly that almost all of the sidebands observed in the measured spectrum are indeed predicted by the model. It further shows that the model's capability in including the gear manufacturing errors is sound. With this, it can be stated that the proposed model can be used to study sideband behavior of planetary gear sets with or without manufacturing errors in both resonance and off-resonance regions of operation. 


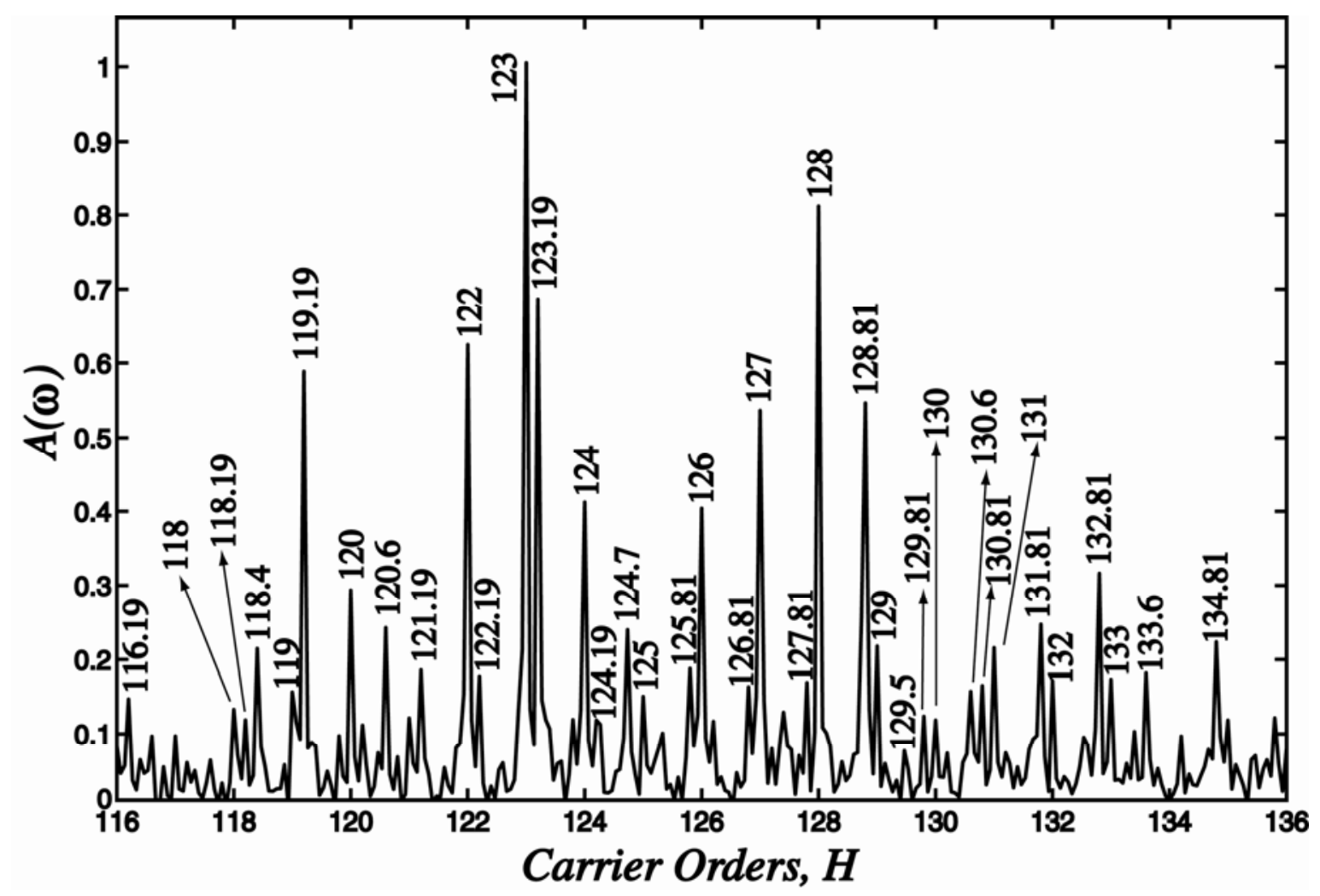

Figure 4.17: A measured $A(\omega)$ spectrum from a 4-planet planetary gear set at $T_{S}=400 \mathrm{Nm}$ and $\Omega_{s}=500 \mathrm{rpm}$. 


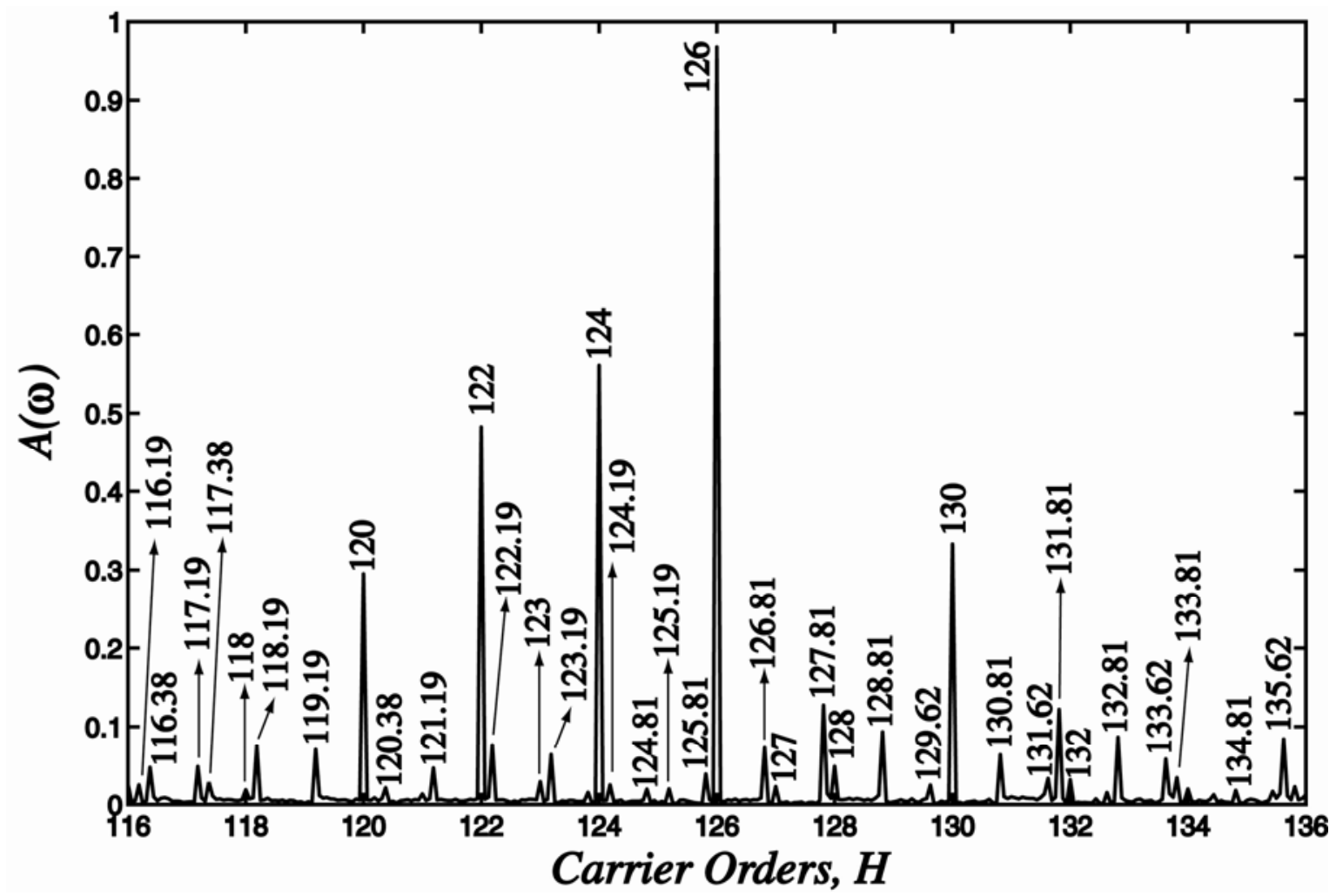

Figure 4.18: Predicted $A(\omega)$ spectrum for the same gear set as in Fig. 4.17 with AM only. 


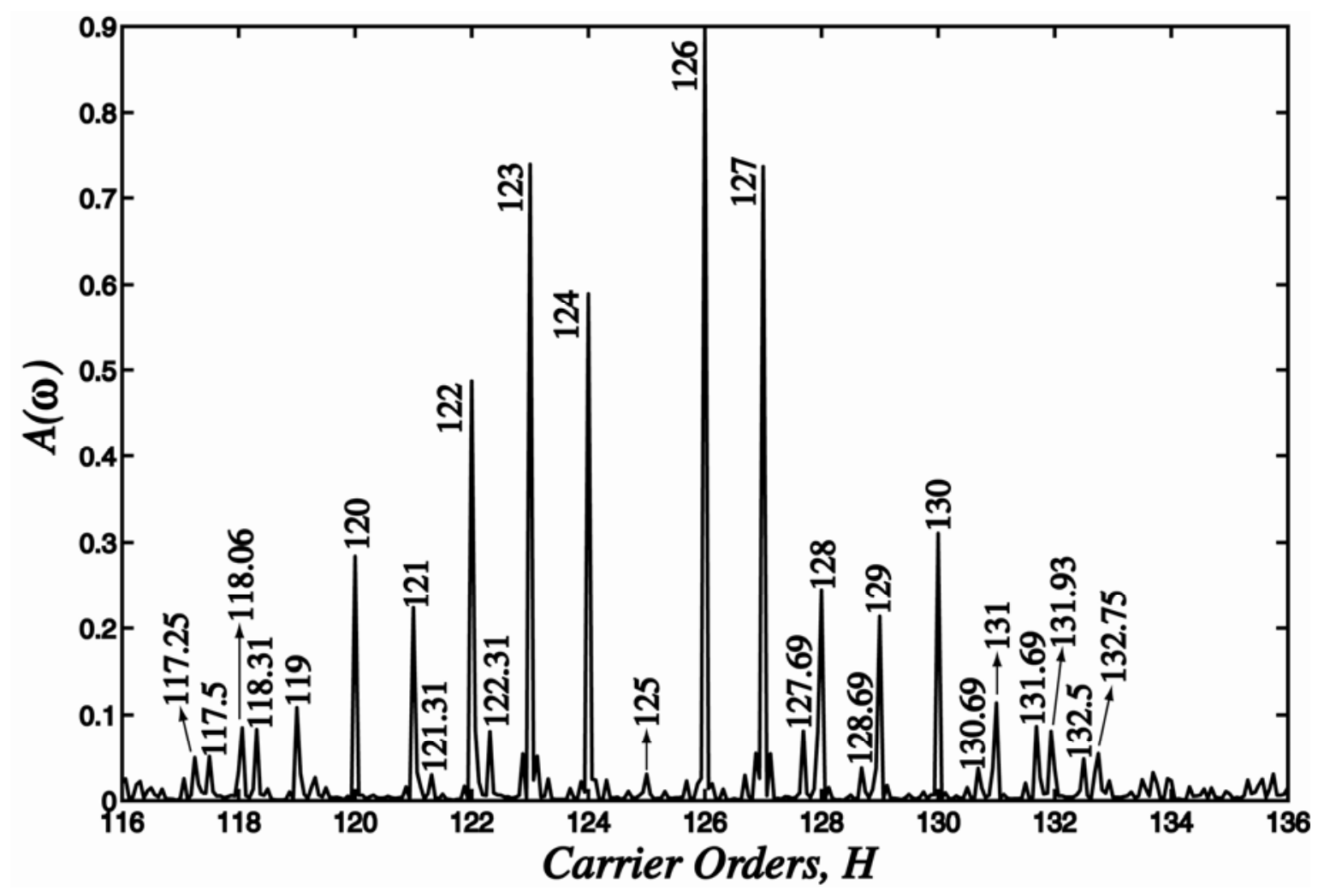

Figure 4.19: Predicted $A(\omega)$ spectrum for the same gear set as in Fig. 4.17 with FM only. 


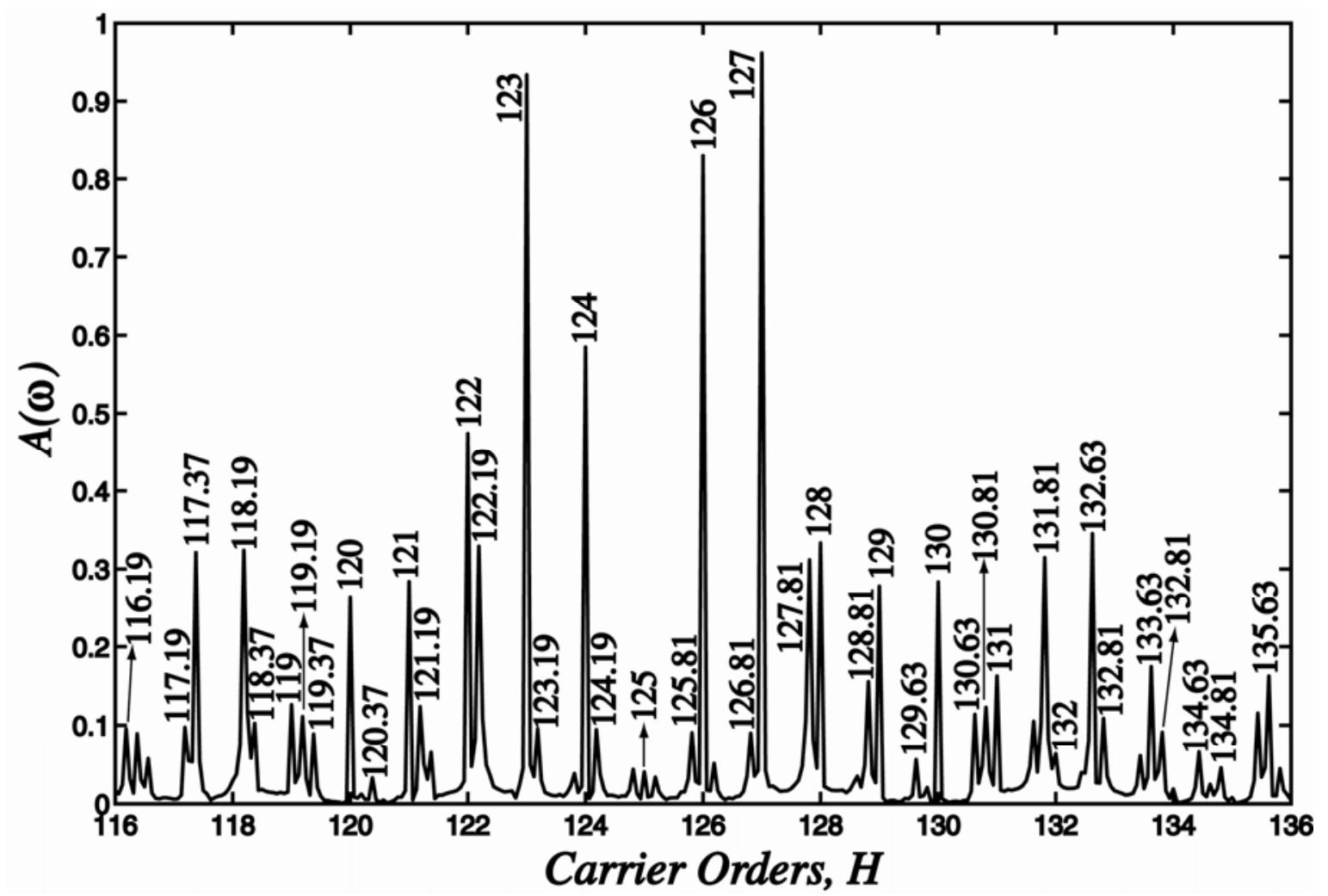

Figure 4.20: Predicted $A(\omega)$ spectrum for the same gear set as in Fig. 4.17 with both AM and FM. 


\subsection{Conclusions}

A dynamic model was proposed in this chapter to predict modulation sidebands of planetary gear sets due to gear manufacturing errors in the forms of run-out or eccentricity, as well as change of the instantaneous gear mesh locations relative to a fixed measurement location due to the rotation of the planet carrier. The dynamic model included periodically time-varying gear mesh stiffnesses and any non-linearities associated with tooth separations. The mesh-frequency excitations were derived in their amplitude and phase modulated forms. The resultant dynamic gear mesh spectra were shown to contain sidebands due to these errors. These modulated dynamic gear mesh force spectra were then used in the simplified analytical formulation of Chapter 3 to include the changes caused by the rotation of the carrier. Predicted acceleration spectra were shown to contain three well-defined groups of sidebands $H_{m} \pm H_{p / c} \pm n$, $H_{m} \pm H_{r / c} \pm n$ and $H_{m} \pm H_{s / c} \pm n$ ( $n$ : integer) due to amplitude modulations associated with the errors of the planets, the ring gear and the sun gears, respectively, while the frequency modulations resulted in more complex sideband structures within wider frequency bands around the fundamental gear mesh frequency. At the end, comparisons to a measured planetary acceleration spectrum were provided to demonstrate that the proposed mechanisms are capable of describing the measured sideband behavior. 


\section{References for Chapter 4:}

[4.1] A. Kahraman, G. W. Blankenship, Planet mesh phasing in epicyclic gear sets, Proceedings of International Gearing Conference, Newcastle UK, September 1994, pp. 99-104.

[4.2] A. Kahraman, Planetary gear train dynamics, ASME Journal of Mechanical Design 116 (1994) 713-720.

[4.3] F. Chaari, T. Fakhfakh, R. Hbaieb, J. Louati, M. Haddar, Influence of manufacturing errors on the dynamic behavior of planetary gears, Int. Journal of Manufacturing Technology 27 (2006) 738-746.

[4.4] W. D. Mark, J. A. Hines, Stationary transducer response to planetary-gear vibration excitation with non-uniform planet loading, Mechanical Systems and Signal Processing, (2008) doi: 10.1016/j.ymssp.2008.09.010.

[4.5] R. B. Randall, A new method of modeling gear faults, Journal of Mechanical Design 104 (1982) 259-267.

[4.6] P. J. Sweeney, R. B. Randall, Sources of gear signal modulation, Second International Conference on Gearbox Noise, Vibration, and Diagnostics, IMechE Conference Transactions, vol. 5, London, England, 1995, pp. 183-198.

[4.7] G. W. Blankenship, R. Singh, Analytical solution for modulation sidebands associated with a class of mechanical oscillators, Journal of Sound and Vibration 179 (1) (1995) 13-36. 
[4.8] A. L. Gu, R. H. Badgley, T. Chiang, Planet-pass-induced vibration in planetary reduction gears, ASME Design Engineering Technical Conference, New York NY USA, October 1974, Paper No: 74-DET-93.

[4.9] A. L. Gu, R. H. Badgley, Prediction of vibration sidebands in gear meshes, ASME Design Engineering Technical Conference, New York NY USA, October 1974, Paper No: 74-DET-95.

[4.10] A. M. Mitchell, F. B. Oswald, and H. H. Coe, Testing of UH-60A helicopter transmission in NASA Lewis $2240 \mathrm{~kW}$ (3000 hp) Facility, NASA Technical Paper 2626, August 1986.

[4.11] M. Mosher, Results from a new separation algorithm for planetary gear system vibration measurements, Proceedings of ASME International Design Engineering Technical Conferences and Computers and Information in Engineering Conference, California USA, September 2005, Paper No: DETC-84404.

[4.12] M. Mosher, Understanding vibration spectra of planetary gear systems for fault detection, Proceedings of ASME Design Engineering Technical Conferences, Illinois USA, September 2003, Paper No: PTG-48082.

[4.13] D. G. Lewicki, J. J. Coy, Vibration characteristics of OH-58A helicopter main rotor transmission, NASA Technical Paper 2705, 1987.

[4.14] A. Saxena, B. Wu, and G. Vachtsevanos, A methodology for analyzing vibration data from planetary gear systems using complex morlet wavelets, 2005 American Control Conference, Portland OR USA, June 2005, pp. 4730-4735. 
[4.15] D. M. Blunt, J. A. Keller, Detection of a fatigue crack in a UH-60A planet gear carrier using vibration analysis, Mechanical Systems and Signal Processing, 20 (2006) 2095-2111.

[4.16] J. A. Hines, D. S. Muench, J. A. Keller, A. K. Garga, Effects of timesynchronous averaging implementations on HUMS features for UH-60A planetary carrier cracking, American Helicopter Society 61st Annual Forum, Grapevine TX USA, June 2005, Paper No: VA 22314-2538.

[4.17] J. A. Keller, P. Grabill, Vibration monitoring of UH-60A main transmission planetary carrier fault, American Helicopter Society 59th Annual Forum, Phoenix Arizona USA, May 2003.

[4.18] H. Ligata, A. Kahraman, A. Singh, A closed-form planet load sharing formulation for planetary gear sets using a translational analogy, Journal of Mechanical Design, 131 (Feb 2009) 021007-1 - 021007-7.

[4.19] A. Kahraman, H. Ligata, K. Kienzle, D. M. Zini, A kinematics and power flow analysis methodology for automatic transmission planetary gear trains, Journal of Mechanical Design, 126 (Nov 2004) 1071-1081.

[4.20] A. Kahraman, Load sharing characteristics of planetary transmissions, Mechanism and Machine Theory, 29-8 (1994) 1151-1165.

[4.21] Gear Load Distribution Program (LDP), The Ohio State University, Columbus, Ohio (2009). 


\section{CHAPTER 5}

\section{CONCLUSIONS AND RECOMMENDATIONS}

\subsection{Summary}

In this study, modulation sidebands of planetary gear sets were investigated both theoretically and experimentally. Considering a general $N$-planet planetary gear set as the basic system, a two-step theoretical study was performed to predict modulations associated with planetary gear sets. In the first step, one of the most obvious causes of sidebands, namely amplitude modulations acquired at a fixed measurement location due to rotating planet carrier and gear meshes, was tackled through simplified, semianalytical manner. This model was designed to produce amplitude modulation sidebands due to $N$ number of known rotating periodic gear mesh forces with predefined phasing relationships. While simple, this model was able to provide the framework to identify key parameters that dictate the modulations of this kind. These parameters were identified to be the same ones that define the phasing relations amongst the planet meshes as well (numbers of teeth of gears, number of planets $N$ and spacing angles of planet). This semi-analytical model was also used to show that planetary gear sets can be classified in five distinct groups based on their sideband behavior: (i) gear sets with equally-spaced and in-phase planets, (ii) gear sets with equally-spaced and sequentially 
phased planets, (iii) gear sets with unequally-spaced and in-phase planets, (iv) gear sets with unequally-spaced and sequentially phased planets, and (v) gear sets with unequallyspaced and arbitrarily phased planets. Planetary gear sets from each class were shown to follow different sideband patterns with well-defined rules.

The second step in the modeling effort focused on predicting modulation sidebands due to various manufacturing errors associated with gears. For this purpose, a discrete $3 N+9$ degree of freedom dynamic model of a planetary gear set was proposed. The model, in addition to the parameters deemed critical in the first model, takes the periodically time-varying mesh stiffnesses and any nonlinearities into account. The excitations for this model were formulated such that they were amplitude and frequency modulated to account for the manufacturing error of gears forming the gear set. With such modulated excitations, predicted dynamic gear mesh force spectra were shown to exhibit sets of sidebands, each associated with the rotational frequency of a gear relative to the planet carrier. These sidebands were further amplitude modulated due to the rotation of the carrier to predict complex, yet well-structured vibration sideband behavior at fixed measurement locations near a planetary gear set.

A separate experimental study was designed and conducted as part of this investigation to (i) build a database of modulation sidebands collected from different classes of planetary gear sets operating at wide ranges of speed and torque conditions, and (ii) provide comparisons to the predictions for validation of the theoretical formulations proposed. The measured spectra contained two groups of sidebands at integer and non-integer orders. The models were shown to predict the measured 
sidebands in vibration spectra effectively. They further demonstrated that the measured modulation sidebands are often due to combination of amplitude modulations due to the rotating carrier and amplitude and frequency modulations of the dynamic gear mesh forces caused by gear manufacturing errors.

\subsection{Conclusions and Contributions}

While several conclusions were made in previous chapters in regards to the sideband behavior of planetary gear sets, some of those major conclusions will be compiled below to provide a concise list of findings. Starting with the experimental study, measurements provided in this thesis, without any need for a theoretical justification, demonstrated indisputably that sidebands are not exceptions, but they are essential components in any vibration spectrum measured around a rotating planetary gear set. These measurements indicated the following general trends:

(i) A subset of sidebands occur at integer orders around a gear mesh harmonic order, i.e., at $H_{m} \pm n$. As $n=1$ represents the order for a complete carrier rotation, these sidebands are related to the modulations caused by a rotating carrier.

(ii) There are additional sidebands that exist at non-integer orders and these sidebands appear at orders that are related to the rotational frequencies of the gears relative to the carrier, indicating that they are due to the manufacturing errors present in the gears. 
(iii) Planetary gear sets having different planet phasing and spacing arrangements were shown to result in sidebands with large amplitudes at different $H_{m} \pm n$ orders.

(iv) The amplitude of the fundamental gear mesh order $H_{m}$ was also shown to be impacted by the planet phasing and spacing conditions. Some spectra did not include any $H_{m}$ component.

The proposed models provided mechanisms to describe the experimentally observed behavior quantitatively. One of these mechanisms to form sidebands, the amplitude modulations due to the carrier rotation were described through the analytical model proposed in Chapter 3. Based on the results of this model, conclusions were derived for integer-order sidebands:

(i) Based on their sideband behavior, planetary gear sets can be classified in one of five distinct groups as: (i) equally-spaced and in-phase planets, (ii) equally-spaced and sequentially phased planets, (iii) unequally-spaced and in-phase planets, (iv) unequally-spaced and sequentially phased planets, and (v) unequally-spaced and arbitrarily phased planets. Gear sets from each category generate different sideband structures when their carrier is not fixed.

(ii) In a sequentially-phased planetary gear set, sidebands are always asymmetrically distributed about the mesh orders. 
(iii) A dominant (and maximum) mesh harmonic amplitude is achieved only when the planet meshes are in phase.

(iv) Symmetric sidebands in both frequencies and amplitudes about the mesh frequency (order) are possible only when the gear set is in-phase and equallyspaced.

The other main mechanism was identified in this study as the manufacturing errors of gears in the form of eccentricity, pitch-line run-out, spacing and indexing errors, all of which cause modulation of both amplitudes and frequencies of the gear mesh excitations. The proposed dynamic model of a planetary gear set, which considers these excitations predicted, was shown to predict gear mesh forces with sets of sidebands to correspond to these errors. When exercised with the carrier rotation formulation, the dynamic model was shown to predict the remaining non-integer order sidebands of measured spectra accurately. The following conclusions can be drawn from the predictions and experimental results presented in Chapter 4:

(i) Predicted acceleration spectra were shown to contain three well-defined groups of sidebands $H_{m} \pm H_{p / c} \pm n, H_{m} \pm H_{r / c} \pm n$ and $H_{m} \pm H_{s / c} \pm n$ (n: integer) due to amplitude modulations associated with the errors of the planets, the ring gear and the sun gear, respectively. However, existence of any particular sideband $H_{m} \pm H_{j / c} \pm n(j=s, r, p i)$ in any spectra depends on its phase and strength.

(ii) Frequency modulations resulted in more complex sideband structures within wider frequency bands around the fundamental gear mesh frequency. 
(iii) Manufacturing errors cause some of the system parameters such as mesh stiffness and transmission error to fluctuate periodically at frequencies directly related to gear relative rotational frequencies.

In view of these conclusions, the main contributions of this research to the stateof-the-art can be listed as follows:

(i) This study provided the first extensive experimental database for the analysis of sideband behavior of planetary gear sets, including three of the five different classes of gear sets under various manufacturing error and operating conditions. This database will be instrumental in validating and guiding ongoing and future modeling efforts.

(ii) This study provided the first simplified mathematical model to predict the modulation sidebands of planetary gear sets due to a rotating carrier. This model also makes it possible to predict vibration and noise spectra measured at a location having a relatively different rotational speed than the carrier.

(iii) This study provided the first general dynamic model of planetary gear sets that can be used to simulate the sideband behavior of planetary gear sets with manufacturing errors as well as a rotating carrier.

(iv) The formulation proposed to describe the interactions between the shaftfrequency excitations due to manufacturing errors and the mesh-frequency excitations (transmission error and gear mesh stiffness functions) provides the first plausible explanation of non-integer order sidebands. 


\subsection{Recommendations for Future Work}

As mentioned in Chapter 2, the experimental database formed as a result of the test program included a variety of conditions. The portion of the database used in this thesis was limited to gear sets having tightly-controlled tolerances and relatively rigid gear blanks. This study was limited to these ideal conditions so that the sideband phenomenon can be described as a function of basic design parameters, kinematic configurations and operating conditions. Also included in this database were spectra collected from gear sets having intentional carrier pin-hole position errors to cause severe unequal planet load sharing situations. Likewise, different ring gears having different rim thicknesses were also included in the test matrix to capture the impact of ring gear deflections on gear set sidebands.

As an example of these different gear set conditions, consider a 3-planet gear set with a flexible ring gear having $\Gamma=0.0025$ (instead of the $\Gamma=0.083$ used in experiments considered in Chapters 3 and 4). This particular ring gear with significantly reduced rim thickness was shown to exhibit large hoop deflections [5.1]. The measured acceleration spectrum shown in Figure 5.1(a) was obtained by using this thin ring gear and an errorless carrier holding 3 equally-spaced planets at $T_{S}=500 \mathrm{Nm}$ and $\Omega_{S}=400$ rpm. The spectrum contains additional large amplitude components away from the main harmonic orders and their associated sidebands (between the $5^{\text {th }}$ and $6^{\text {th }}$ mesh harmonic order due to a ring gear natural frequency at $f_{n} \approx 1660 \mathrm{~Hz}$. When input speed was increased to $\Omega_{s}=442 \mathrm{rpm}$, such that $f_{m}=332 \mathrm{~Hz}$ and $f_{n} \approx 5 f_{m}$, the fifth gear mesh harmonic order and its sidebands increase their amplitude as shown in Figure 5.1(b) 
similarly, in Figure 5.1(c) for $\Omega_{s}=553 \mathrm{rpm}\left(f_{m}=425 \mathrm{~Hz}\right.$ and $\left.f_{n} \approx 4 f_{m}\right)$, the $4^{\text {th }}$ gear mesh order is excited by this mode. Similarly, in Figures 5.1(d) to (f) for $\Omega_{s}=737$, 1106, and $2213 \mathrm{rpm}$, respectively, resonances at $f_{n} \approx 3 f_{m}, f_{n} \approx 2 f_{m}$, and $f_{n} \approx f_{m}$ are evident, illustrating the influence of ring gear flexibility. This behavior, for instance, could not be predicted by the proposed discrete models. There are several other parameters and conditions that can impact the planetary gear set spectra. A logical extension to this study would be adding capabilities to include such parameters or conditions. Specifically:

- A deformable-body dynamic model of the planetary gear sets can be used in place of the discrete model to include the gear rim deflections in addition to defining the excitations with to manufacturing errors implicitly.

- A similar deformable body model can be used to define force transmission paths more accurately.

- $\quad$ A load sharing formulation can be embedded to the current dynamic model to predict the sideband amplitudes more precisely taking the unequal load sharing effects into account.

- $\quad$ The 2D discrete dynamic model proposed in Chapter 4 can be replaced by a 3D equivalent to study sideband behavior unique to helical planetary gear sets.

- The modulation prediction models developed in this dissertation can be expanded to study the modulation characteristics of multi-stage planetary gear sets as well double-helical planetary gear sets. 
- The experimental database can be complemented by the corresponding noise data to explore the impact of planetary sidebands on sound quality aspects of gearboxes. 

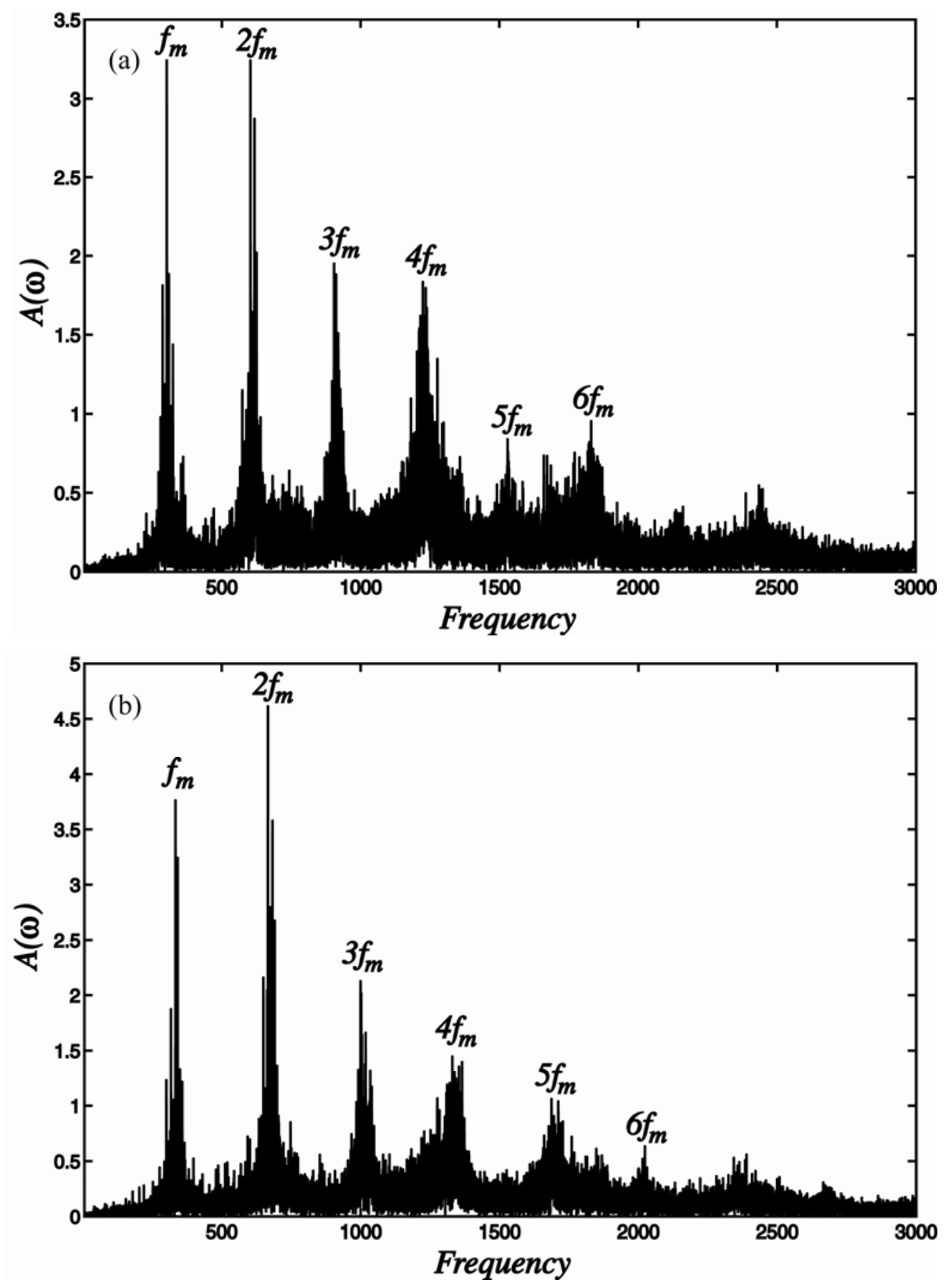

Continued

Figure 5.1: Measured broadband acceleration spectrum for a three planet gear set using a thin ring gear ( $\Gamma=0.0025)$ at $T_{s}=500 \mathrm{Nm}$ and (a) $\Omega_{s}=400 \mathrm{rpm}$, (b) $\Omega_{s}=442 \mathrm{rpm}$, (c) $\Omega_{s}=553 \mathrm{rpm}$, (d) $\Omega_{s}=737 \mathrm{rpm}$, (e) $\Omega_{s}=1106 \mathrm{rpm}$, and (f) $\Omega_{s}=2213 \mathrm{rpm}$. 
Figure 5.1: Continued.
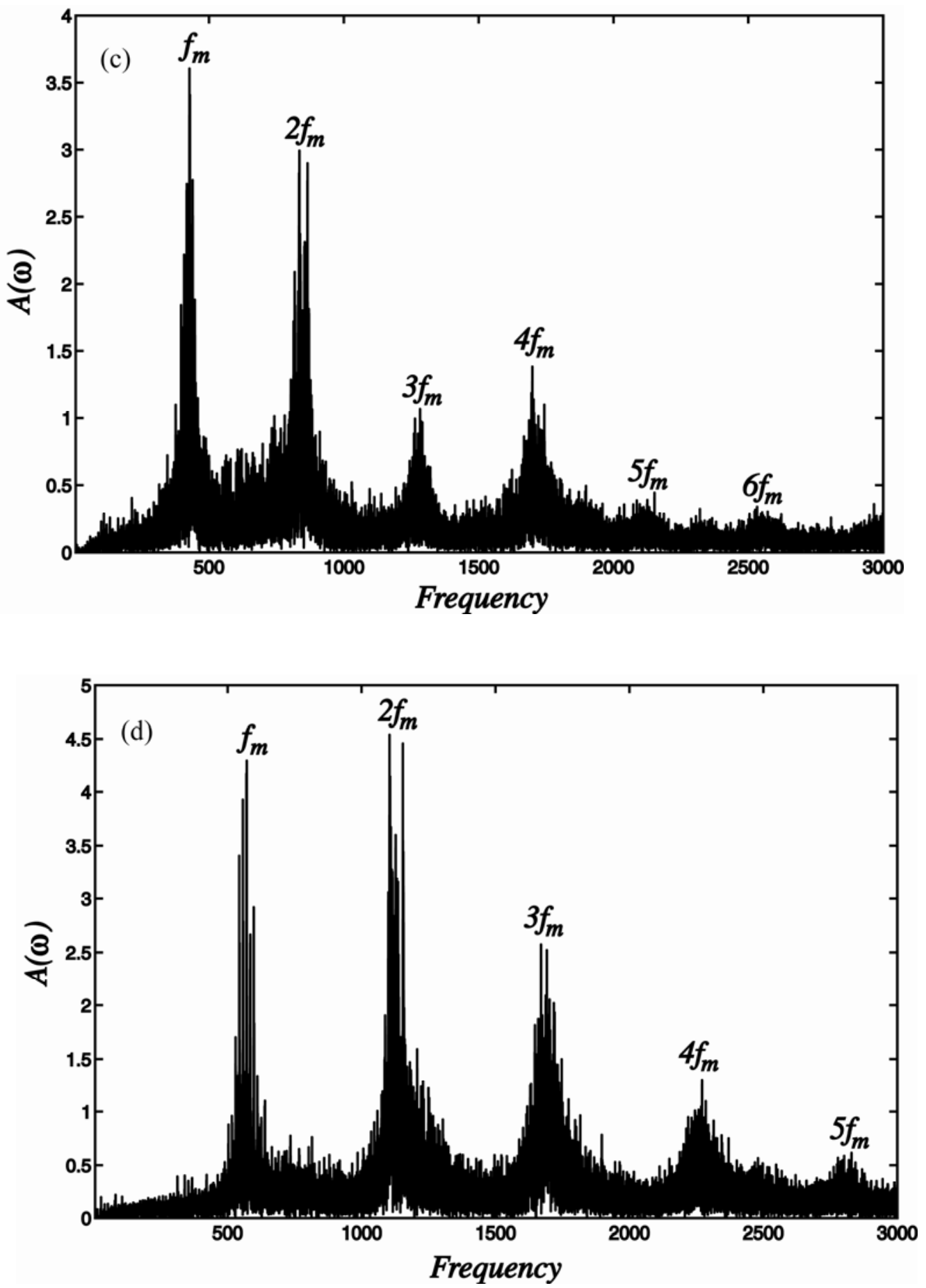

Continued 
Figure 5.1: Continued.
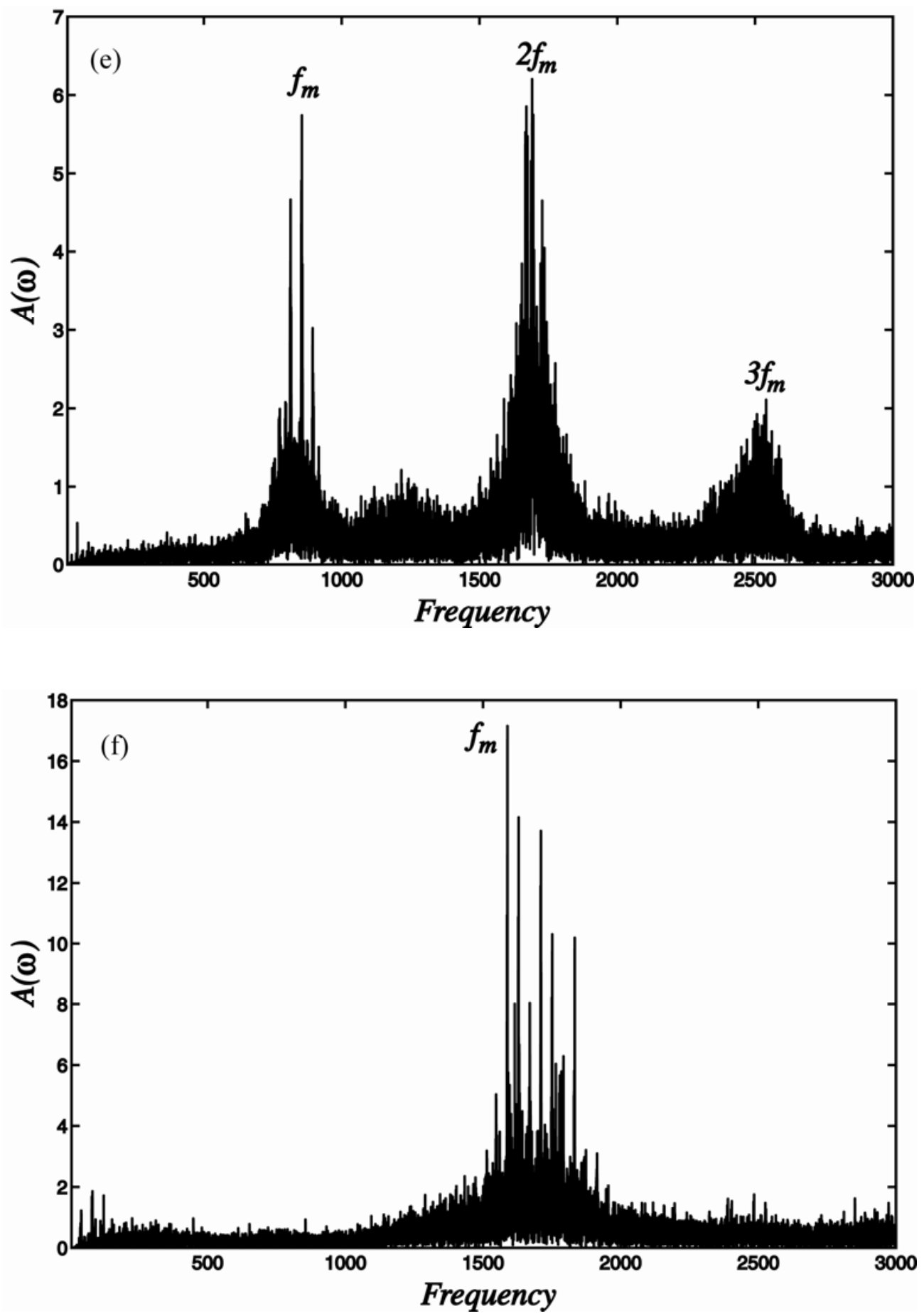


\section{References for Chapter 5:}

[5.1] H. Ligata, A. Kahraman, and A. Singh, An experimental study on the influence of manufacturing errors on the planetary gear stresses and planet load sharing, ASME Journal of Mechanical Design, 130 (4) (2008) 041701-1 - 041701-9. 


\section{BIBLIOGRAPHY}

A. Al-shyyab, A. Kahraman, A non-linear dynamic model for planetary gear sets, Proceedings of the Institution of Mechanical Engineers Part K: Journal of Multi-body Dynamics, 221 (2007) 567-576.

L. Bellomonte, I. Guastella, R. M. Sperandeo-Mineo, Mechanical models of amplitude and frequency modulation, European Journal of Physics, 26 (2005) 409-422.

G. W. Blankenship, R. Singh, Analytical solution for modulation sidebands associated with a class of mechanical oscillators, Journal of Sound and Vibration, 179 (1) (1995) 13-36.

D. M. Blunt, J. A. Keller, Detection of a fatigue crack in a UH-60A planet gear carrier using vibration analysis, Mechanical Systems and Signal Processing, 20 (2006) 20952111.

D. M. Blunt, J. A. Keller, Detection of a fatigue crack in a UH-60A planet gear carrier using vibration analysis, Mechanical Systems and Signal Processing, 20 (2006) 20952111.

A. Bodas, A. Kahraman, Influence of carrier and gear manufacturing errors on the static load sharing behavior of planetary gear sets, JSME International Journal, Series C, 47-3 (2004) 908-915.

M. Botman, Vibration measurements on planetary gears of aircraft turbine engines, Journal of Aircraft, 17 (5) (1980) 79 - 7012R.

F. Chaari, T. Fakhfakh, R. Hbaieb, J. Louati, M. Haddar, Influence of manufacturing errors on the dynamic behavior of planetary gears, Int. Journal of Manufacturing Technology 27 (2006) 738-746. 
C. E. Fair, Synchronous sampling sideband orders from helical planetary gear sets, MS Thesis, Virginia Polytechnic Institute and State University, 1998.

Gear Load Distribution Program (LDP), The Ohio State University, Columbus, Ohio (2009).

A. L. Gu, R. H. Badgley, T. Chiang, Planet-pass-induced vibration in planetary reduction gears, ASME Design Engineering Technical Conference, New York NY USA, October 1974, Paper No: 74-DET-93.

A. L. Gu, R. H. Badgley, Prediction of vibration sidebands in gear meshes, ASME Design Engineering Technical Conference, New York NY USA, October 1974, Paper No: 74DET-95.

T. Hidaka, Y. Terauchi, K. Ishioka, Dynamic behavior of planetary gear $\left(2^{\text {nd }}\right.$ report: displacement of sun gear and ring gear), Bulletin of the JSME, 19-138 (1976) 1563-1570.

J. A. Hines, D. S. Muench, J. A. Keller, A. K. Garga, Effects of time-synchronous averaging implementations on HUMS features for UH-60A planetary carrier cracking, American Helicopter Society 61st Annual Forum, Grapevine TX USA, June 2005, Paper No: VA 22314-2538.

M. Inalpolat, A. Kahraman, Dynamic modelling of planetary gears of automatic transmissions, Proceedings of the Institution of Mechanical Engineers Part K: Journal of Multi-body Dynamics, 222 (2008) 229-242.

A. Kahraman, G. W. Blankenship, Planet mesh phasing in epicyclic gear sets, Proceedings of International Gearing Conference, Newcastle UK, September 1994, pp. 99-104.

A. Kahraman, Planetary gear train dynamics, ASME Journal of Mechanical Design 116 (1994) 713-720. 
A. Kahraman, Load sharing characteristics of planetary transmissions, Mechanism and Machine Theory, 29-8 (1994) 1151-1165.

A. Kahraman, H. Ligata, K. Kienzle, D. M. Zini, A kinematics and power flow analysis methodology for automatic transmission planetary gear trains, Journal of Mechanical Design, 126 (Nov 2004) 1071-1081.

J. A. Keller, P. Grabill, Vibration monitoring of UH-60A main transmission planetary carrier fault, American Helicopter Society 59th Annual Forum, Phoenix Arizona USA, May 2003.

D. G. Lewicki, J. J. Coy, Vibration characteristics of OH-58A helicopter main rotor transmission, NASA Technical Paper 2705, 1987.

H. Ligata, A. Kahraman, A. Singh, An experimental study of the influence of manufacturing errors on the planetary gear stresses and planet load sharing, ASME Journal of Mechanical Design, 130 (4) (2008) 041701-1 - 041701-9.

H. Ligata, A. Kahraman, A. Singh, A closed-form planet load sharing formulation for planetary gear sets using a translational analogy, Journal of Mechanical Design, 131 (Feb 2009) 021007-1 - 021007-7.

H. Ligata, Impact of system-level factors on planetary gear set behavior, Ph.D. Thesis, The Ohio State University, 2007.

P. Lynwander, Gear Drive Systems, Marcel Dekker Inc., New York, 1983.

W. D. Mark, J. A. Hines, Stationary transducer response to planetary-gear vibration excitation with non-uniform planet loading, Mechanical Systems and Signal Processing, (2008) doi: 10.1016/j.ymssp.2008.09.010. 
P. D. McFadden, J. D. Smith, An explanation for the asymmetry of the modulation sidebands about the tooth meshing frequency in epicyclic gear vibration, Proceedings of the Institution of Mechanical Engineers 199 C1 (1985) 65-70.

J. McNames, Fourier series analysis of epicyclic gearbox vibration, ASME Journal of Vibration and Acoustics, 124 (2002) 150-152.

A. M. Mitchell, F. B. Oswald, H. H. Coe, Testing of UH-60A helicopter transmission in NASA Lewis 2240 kW (3000 hp) Facility, NASA Technical Paper 2626, August 1986.

M. Mosher, Understanding vibration spectra of planetary gear systems for fault detection, Proceedings of ASME Design Engineering Technical Conferences, Illinois USA, September 2003, Paper No: PTG-48082.

M. Mosher, Results from a new separation algorithm for planetary gear system vibration measurements, Proceedings of ASME International Design Engineering Technical Conferences and Computers and Information in Engineering Conference, California USA, September 2005, Paper No: DETC-84404.

W. H. Muller, Epicyclic Drive Trains, Wayne State University Press, Detroit, 1982.

R. Patrick-Aldaco, A model based framework for fault diagnosis and prognosis of dynamical systems with an application to helicopter transmissions, Ph.D. Thesis, Georgia Institute of Technology, August 2007.

R. B. Randall, A new method of modeling gear faults, Journal of Mechanical Design 104 (1982) 259-267.

A. Saxena, B. Wu, G. Vachtsevanos, A methodology for analyzing vibration data from planetary gear systems using complex morlet wavelets, 2005 American Control Conference, Portland OR USA, June 2005, pp. 4730-4735. 
P. P. Schon, Unconditionally convergent time domain adaptive and time-frequency techniques for epicyclic gearbox vibration, ME Thesis, The University of Pretoria, June 2005.

A. Singh, A. Kahraman, H. Ligata, Internal gear strains and load sharing in planetary transmissions - model and experiments, ASME Journal of Mechanical Design, 130 (7) (2008) 072602-1 - 072602-10.

P. J. Sweeney, R. B. Randall, Sources of gear signal modulation, Second International Conference on Gearbox Noise, Vibration, and Diagnostics, IMechE Conference Transactions, vol. 5, London, England, 1995, pp. 183-198. 